MAP LJB.

$G A$

YOUivo

SOME MUESTIGA-

TIONS IN THE

THEORY OF MAP

PROJECTIONS

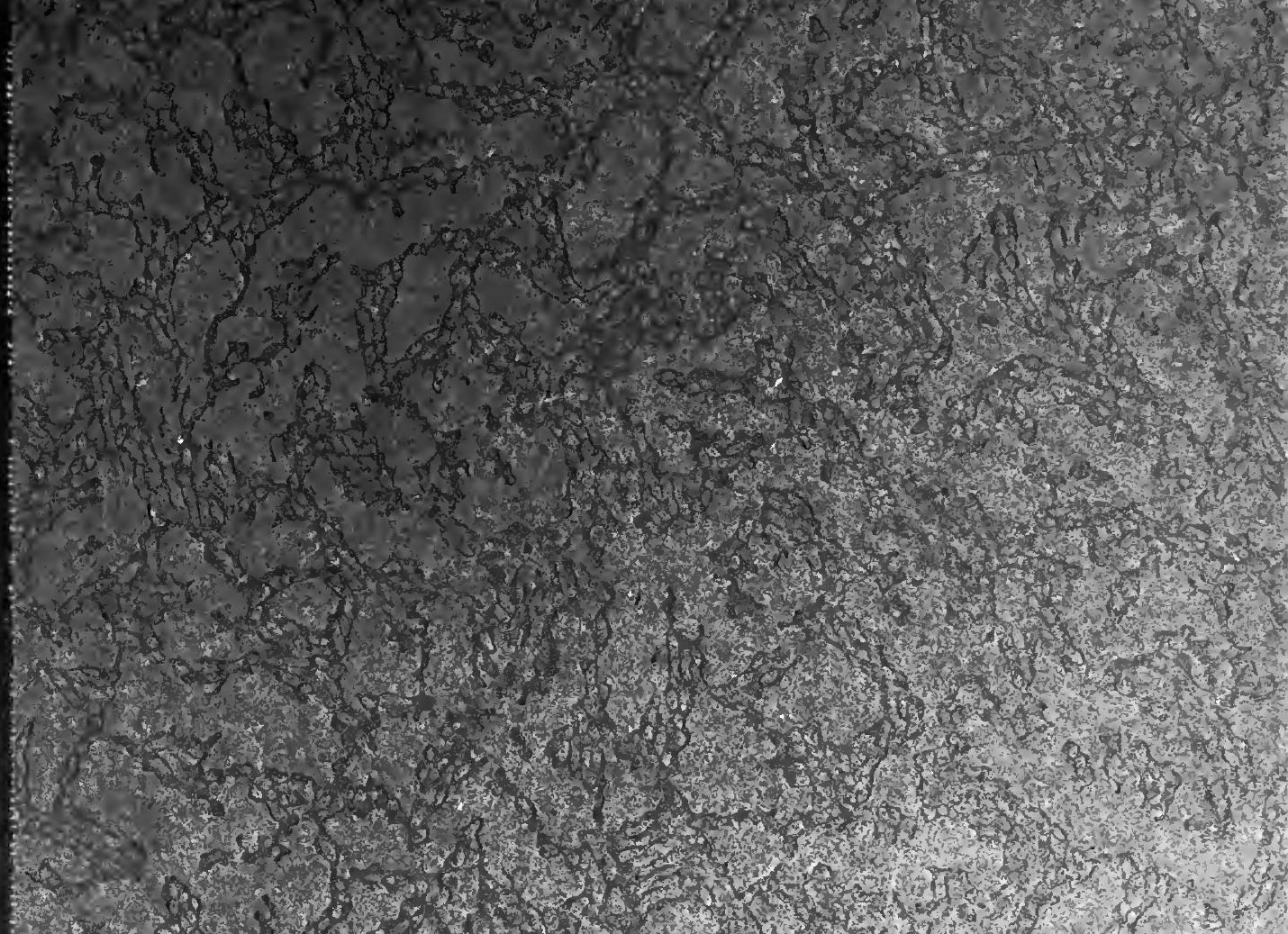

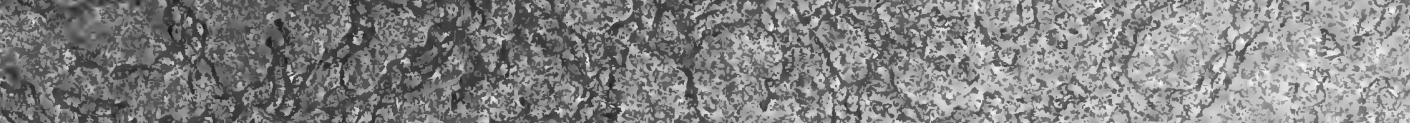

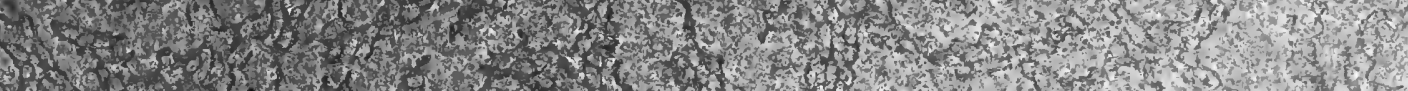

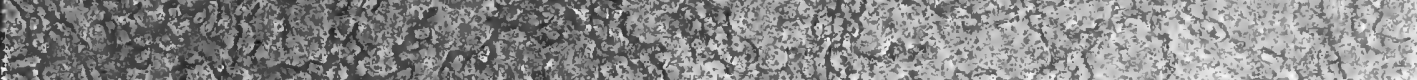

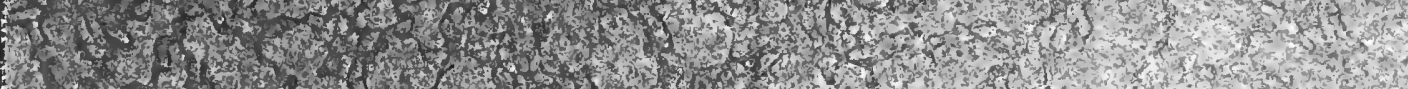

(1)
(1) (1) (1) 6.

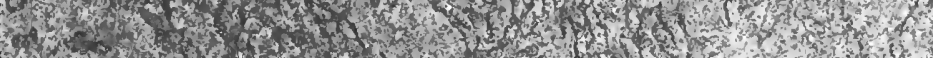




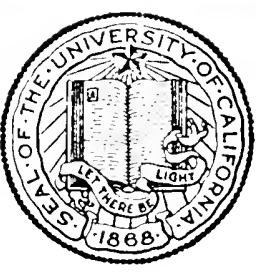

\section{THE LIBRARY OF}

THE UNIVERSITY OF CALIFORNIA LOS ANGELES 
R.G.S. TECHNICAL SERIES: No. I.

SOME INVESTIGATIONS IN THE THEORY OF MAP PROJECTIONS 
The Council of the Royal Geographical Socicty have, on the recommendation of the Rescarch Committec, approved the publication of this work as the first of a Series designed to include important contributions to Geography too technical and too long for the Goographical Journal.

ARTHUR R. HINKS,

Secretary.

24 September I920. 


\section{SOME INVESTIGATIONS IN THE THEORY OF}

\section{MAP PROJECTIONS}

BY

\section{ALFRED ERNEST YOUNG,}

FELLOW OF THE CITY GUILDS INSTITUTE, ASSOC. MEM. INST.C.E., LATE DEPUTY-SURVEYOR-GENERAI, FEDERATED MALAY STATES

\section{LONDON}

ROYAL GEOGRAPHICAL SOCIETY

KENSINGTON GORE, S.IV.7 


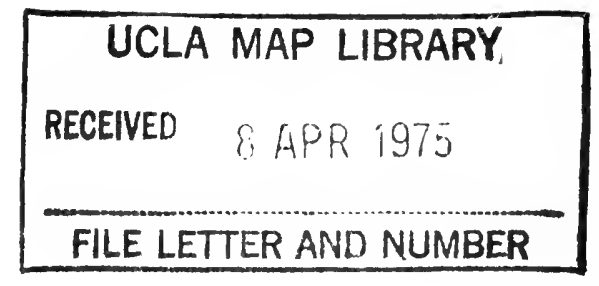




\section{PREFACE}

$\mathrm{T}$

HE Theory of Map Projections has received the attention of so many eminent mathematicians and geodesists that it may seem somewhat presumptuous to offer anything further on the subject. These Papers must therefore prove their own justification. They are the result of investigations extending over (with intervals) nearly four years, by one who was for twenty years employed on the Trigonometrical and General Survey of one of the British Protectorates in Tropical Asia, but who had until recently clevoted little attention to the theory of map projections, being content to follow the established methods of the Ordnance Survey and the Survey of India.

The investigations came about in this way. At the beginning of 1916 the writer had offered his services to the Secretary of the Royal Geographical Society to assist in the map work being done by that Society for the IVar Office. One of the first tasks given him by Mr Hinks was to compute some Tables of rectangular coordinates of the special modified Polyconic Projection adopted for the International Map on the scale of I million, for a few sheets to be compiled on half that scale. The carrying out of this calculation led to an examination of the basis of the formulac on which the modification referred to depends. This led to a comparison with other Projections and their errors, until, becoming completely fascinated with the subject, the writer thinks he can claim to have

Q passed under review the principal books and Papers which have been published on it, and also to have worked out some results which are new so far as he knows, and which he hopes may prove useful to practical map-makers and surveyors. These results are concerned mostly with the Minimum Error Projections, and although the writer cannot claim to have discovered anything of striking novelty in either theory or practice, he has been able to extend 
the method invented by Airy and Clarke more generally, and to simplify their results; so that, out of the almost bewildering number of Projections that have been discovered and advocated from time to time, those which are practically the best or most useful are reduced to comparatively few, the application of which has becn simplified. One of the most interesting results is to establish in their proper place some old and, by later writers, somewhat disparaged Projections, which seem to have been hit upon by their inventors by a sort of geometrical intuition rather than by rigorous analysis.

Owing to the present high cost of printing the Papers are presented in as concise a form as possible. They do not profess to be a complete treatment of the subject $a b$ initio, but only a statement of what are supposed to be new results. Many steps in the mathematical processes have been left out, and in the last parts it will be difficult to follow the argument without reference to the worlis of the other writers mentioned.

The Tables etc. have been calculated with 5-figure logarithms and Fuller's slide-rule, and are intended only to be illustrative. In the formulae the symbol $\log$ means the natural logarithm and the angles are supposed to be reckoned in circular measure. Thongh the work has been checked where possible by alternative methods, there has been no absolutely independent check, and it cannot be that some errors have not crept in both in formulac and arithmetical work. The writer will therefore welcome corrections from any reader who may have the will and opportunity to check his results.

In the course of these investigations the writer received valuable hints as to suitable and fruitful lines of inquiry from $\mathrm{Mr}$. A. R. I Iink: F.R.S., Secretary, Royal Geographical Society, to whose own little book on Map Projections he is indebted for a clear preliminary survey of the whole subject. He has also received valuable assistance in mathematical difficulties from professor A. L. 11. Lore, L.R.S. Oxford, Dr T. J. I'A. Bromwich, F.R.S., Cambridge, and P'rofersin J. W. Nicholson, F.R.S., King's Colleger London. He is indebted to Captain (r. T. MeCaw, General Staff, for reatling the original draft and for malking some useful susgestions. 
The following is a list of the principal works consulted. When quoted in the text they will be referred to by author's name and the page, e.g. Germain, p. I 50 , for the sake of brevity :

Germain, A. Traité des Projections des Cartes Géographiques, Paris, i 865. Fiorini, M. Le Projezioni delle Carte Geografiche, Bologna, r88 I.

Tissot, A. Mémoire sur la Représentations des Surfaces et les P'rojections des Cartes Géographiques, I’aris, I881.

Craig, Thomas. A Treatise on Map Projections, Washington, 1882.

Herz, Noriert. Lehrbuch der Landkartenprojektionen, Vienna, I885.

Helmert, F. R. Höhere Geodäsie, Vol. I, Leipzig, i 880.

Hammer, E. Über die geographischen wichtigsten Kartenprojektionen, Stuttgart, I 889 .

P'izzetti, P. Trattato di Geodesia Teoretica, Bologna, I905.

Craig, J. I; The Theory of Map Projections, Cairo, igro.

Hinks, A. R. Map Projections, Cambridge, I912.

Adans, Oscar S. Special Publications of United States Coast and Geodetic Survey, Nos. 47, 49, 52, 57, Washington, 1917-19.

Encyclopaedia Britannica. Articles on Nathematical Geography, 9 th Edition, and on Map Projections, i ith Edition.

JORDAN, W: Handbuch der Vermessungskunde, Vol. III, 6th edition, Stuttgart, 1916 .

A. E. YOUNG.

Royal Geograinical Societr,

KENSINGTON GORE, LoxDOA, S.II.7. May' 1920. 


\section{CONTEN'TS}

\section{Chap. I. The Minimum Error Zenithal Projections}

Airy's Zenithal Projection . . . . . . . . . 2

Brensing's l'rojection. . . . . . . . . . . 7

Orthomorphic and Equal Area . . . . . . . . 8

Equidistant . . . . . . . . . . . 9

With total area true. . . . . . . . . . Io

Perspective . . . . . . . . . . . 12

With scale true at centre . . . . . . . . . 17

Evaluation of Average Error . . . . . . . . . 19

Chap. II. The Minimum Error Conical Projections . . 22

Orthomorphic . . . . . . . . . . . 23

Approximately Equal Area . . . . . . . . . 25

Minimum Error Map of a Zone . . . . . . . 26

Radial scale true . . . . . . . . . . . 29

Ilate-Carrée . . . . . . . . . . . . 33

Cassini . . . . . . . . . . . . . 34

Merator . . . . . . . . . . . 34

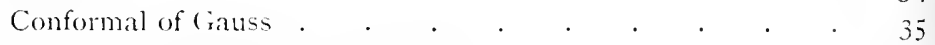

Euler's with two stanclard parallels . . . . . . . 35

Murdoch's Projections . . . . . . . . . . 38

Equiclistant with total area true . . . . . . . 4 .

Albers' Equal Area . . . . . . . . . . . . 45

Collignon's Workl Map . . . . . . . . . . . to

G. W. Mlills l'rojection . . . . . . . . . . . 4 49

Best projection for given comtry . . . . . . . . . + t9

Mathematical paradox . . . . . . . . . . . . 55

Chap. 11I. The Conical (Orthomorphic Projection with two st:undard parallels (I ambert's seconel) for the Spheroid. 57

Chitp. IV. The Prigconic Projections. . . . . 66

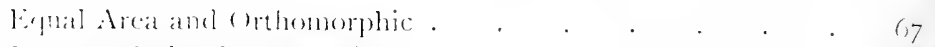

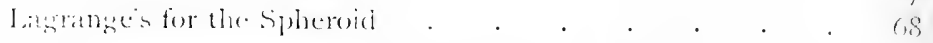

Chate Y. The finite comes of Projections. . . . . forg

(hape Vl. The ('mersency of Meridians. . . . . 7.

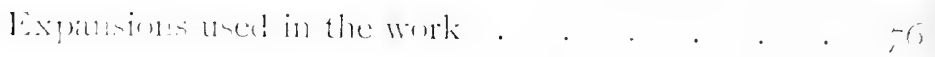




\section{CHAPTER I}

\section{THE MINIMUM ERROR ZENITHAL I'ROJECTIONS}

The Zenithal Projections are a particular case of the Conical Class obtained by opening out the cone until it becomes a plane or, which is the same thing, by making the constant of the cone equal to unity. They will be considered before the general conical class, however, because they are the first to which the principle of Minimum Error was applied, and it will be simpler to approach the subject from what has already been done.

Suppose that $r$ is the distance from the centre or pole to any point on the map, then if $\theta$ is the corresponding angle subtended by this distance at the centre of the Earth, supposed spherical, we have as the law of the projection $r=R f(\theta)$, where $R$ is the radius of the Earth. In the following investigations we shall for simplicity assume $R=$ unity, but it must be remembered that to convert the values of $r$ in any case into actual distances, they must be multiplied by the proper value of $R$ on the appropriate scale.

The radial scale of the map is given at once by $d r / d \theta$ and the perpendicular scale by $r / \sin \theta$.

By choosing different forms of the function $r=f(\theta)$ we obtain Zenithal Projections with different properties of which the following are the principal :

Name

I. Equidistant

2. Orthomorphic or Stereographic

3. Equal Area

4. Gnomonic

5. Perspective

$$
r=f(\theta)
$$

$\theta$

$$
\begin{aligned}
& 2 \tan \frac{1}{2} \theta \\
& 2 \sin \frac{1}{2} \theta \\
& \tan \theta \\
& k \sin \theta \\
& h+\cos \theta
\end{aligned}
$$

$$
\begin{aligned}
& \text { Radial Scale } \\
& =d r / d \theta
\end{aligned}
$$

I

$\sec ^{2}:_{2}^{1} \theta$

$\cos \frac{1}{2} \theta$

$\sec ^{2} \theta$

$k(h \cos \theta+\mathrm{I})$

$(h+\cos \theta)^{2}$
Perpendicular Scale
$=r / \sin \theta$ $\operatorname{cosec} \theta$

$$
\begin{gathered}
\sec ^{2} \frac{1}{2} \theta \\
\sec \frac{1}{2} \theta \\
\sec \theta \\
k \\
h+\cos \theta
\end{gathered}
$$

It will be observed that the fifth only is multiplied by a constant $k$, commonly called a scale constant, but it will be shewn later that the proper evaluation of this constant plays a most important part in the determination of the criterion of minimum error, and that a similar constant could be applied to the other four with like result, which, so far as the writer is aware, has not hitherto been noticed. The second projection in the tible has the property that the scale in any direction round a point is the same, and the third the property that the radial and perpendicular scales at any point are reciprocal, so that equality of small areas round any point is preserved. 


\section{Airy's Zenithal Projection by Balance of Errors.}

Sir George Airy, late Astronomer Royal, conceived the idea of a projection which should be a sort of compromise between these two. His investigation is published in the Philosophical Masasine for December $186 \mathrm{I}$. It consists in finding by mathematical analysis the form of the function $r f(\theta)$ which makes the squares of the two scale errors, integrated over the surface of the sphere represented by the map, a minimum. Airy's method consists in fact of making the average scale error, or more strictly speaking the mean square scale error, of the map a minimum; this averige being found by syuaring the scale errors in the two directions mutually perpendicular, integrating them over that portion of the sphere represented hy the map, then (implicitly) dividing by the area of the same surface and extracting the square root. 'The result will be the mean spuare scale error of the map, from which the average scale error can be found by multiplying by $0.7979 \ldots$

Unless orthomorphism or equality of small areas is wanted for some particular purpose, it is difficult to conceive a more rational criterion than Airy's for finding the best projection. Another criterion is to make the maximum positive scale error equal to the maximum negative scale error, in other words to make the maximum scale error as small as possible. since the scale error increases as the square of the distance from the pole of projection, which is the centre of the map in the zenithal class, this has the effect of diminishing the scale error at the margin of the map at the expense of increasing it near the centre and increasing therefore the average.

Nevertheless, Airy's Projection by Balance of Errors has not been much used, and it scems to have attracted more attention on the Continent than in this country. The reason, probably, is the apparent complexity of its formula, but also, the writer thinks, because its nature has not been fully understood or explained in the text books - the more so since Airy himself made a slip in his solution which caused the projection to ilppear under a disadvantage in the Tables published with it. Although this slip) was discovered by (larke and rectified in a paper published in the Philosofhical Warasine for April 1862, Airy's erroneous tables have unfortunately been incorporated in later publications, e.s. A Treatise on Projections by thomas Craig, United states Coast and (icodetic Survey (Washington, 1882), and others.

It also seems to be implied by some writers that Airy's is the Minimum lirror l'rojection, whereas it is only the Minimum Error l'rojection of the Zeinithal Class, which is a particular case of the more general Conical Class. The wrier has sucreded in applying Airy's method to this more general chass, and the results derived will be given later.

Germain frives a full account of Airy's Projection with Clarke's rorrection and in his gemeral review p. 227 says, "la projection de sir liry, balance

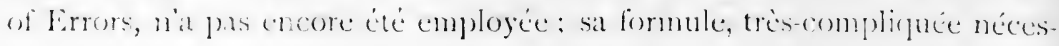


site des calculs très-penibles, et les problèmes de la sphère ne peuvent s'y résoudre facilement." Its qualities and the modification of it called Breusing's Projection are also treated by Hammer; but the writer has found by far the most complete treatment, with some extensions of Airy's and Clarke's method, in 'Le Projezioni delle Carte Geografiche by Professor Matteo Fiorini (Bologna, I 88I), for reference to which he is indebted to a pamphlet by J. I. Craig of the Egyptian Survey Department on The Theory of Map P'rojections (Cairo, I 9 Io).

Returning to Airy's solution, the two scale errors at any zone at angular distance $\theta$ from the centre of map are $d r / d \theta-\mathbf{I}$ and $r / \sin \theta-\mathbf{I}$. The corresponding element of area on the sphere is

$$
R d \theta .2 \pi R \sin \theta=2 \pi R^{2} \sin \theta d \theta,
$$

and the problem therefore is, omitting the constant multiplier $2 \pi R^{2}$, to find the function $r=f(\theta)$ which will make the integral

$$
M=\int_{0}^{\beta}\left\{\left(\frac{d r}{d \theta}-\mathrm{I}\right)^{2}+\left(\frac{r}{\sin \theta}-\mathbf{I}\right)^{2}\right\} \sin \theta d \theta \text { a minimum, }
$$

where $\beta$ is the angular radius at the limit of the map. The corresponding area on the sphere is $2 \pi R^{2} \int_{0}^{\beta} \sin \theta d \theta=\mathrm{I}-\cos \beta$, omitting the constant multiplier $2 \pi R^{2}$; so that the square of the mean square error, which we shall call $\mu^{2}$, is given by $M /(\mathrm{r}-\cos \beta)$. By the Calculus of Variations, Airy finds

$$
r=A \tan \frac{1}{2} \theta+2 \cot \frac{1}{2} \theta \log \sec \frac{1}{2} \theta+B \cot \frac{1}{2} \theta,
$$

where $A$ and $B$ are constants to be determined by the boundary conditions. Since $r$ must $=0$ when $\theta=0$, Airy and Clarke put at once $B=0$. Airy then makes the mistake of supposing that the constant $A$ is completely at his disposal and he chooses $A$ so that the scale error shall vanish at the centre, i.e. he takes $A=\mathbf{I}$, so that

$$
r=\tan \frac{1}{2} \theta+2 \cot \frac{1}{2} \theta \log \sec \frac{1}{2} \theta \text { simply, }
$$

from which his Tables have been calculated. The Calculus of Variations exacts however that at each boundary $d r / d \theta=\mathrm{I}$, and we ought, strictly speaking, to suppose the map to extend from a lower limit $\theta=\alpha$ to a higher limit $\theta=\beta$, at each of which $d r / d \theta=\mathrm{I}$.

From these two equations we should obtain

$$
\begin{aligned}
& A=2\left(\begin{array}{l}
\log \sec \frac{1}{2} \beta-\log \sec \frac{1}{2} a \\
\tan ^{2} \frac{1}{2} \beta-\tan ^{2} \frac{1}{2} \alpha
\end{array}\right): \\
& B=2\left(\frac{\tan ^{2} \frac{1}{2} \alpha \log \sec \frac{1}{2} \beta-\tan ^{2} \frac{1}{2} \beta \log \sec \sin _{2}^{1} \alpha}{\tan ^{2} \frac{1}{2} \beta-\tan ^{2} \frac{1}{2} a}\right) .
\end{aligned}
$$

If then our map extends from the centre we put $\alpha=0$ and find

$$
A=2 \cot ^{2} \frac{1}{2} \beta \log \sec \frac{1}{2} \beta, \quad B=0,
$$

which are the values of the constants found by Clarke. If our map were to extend over a zone of the Earth's surface from a spherical radius a to a spherical radius $\beta$, we should reduce the average scale error ly making use 
of the constant $B$ as calculated above, and the term $B \cot \frac{1}{2} \theta$. It is not likely, however, that such a map would be of much use in the zenithal system, though, as we shall see later, this term is of great importance in the zonal maps of the Conical Class.

Let us now examine the separate terms of Airy's solution. 'The first, $A \tan { }_{2}^{1} \theta$, is evidently half the characteristic formula of the Orthomorphic, $r=2 \tan \operatorname{lar}_{2} \theta$, or it is the solution of the differential equation $d r / d \theta=r / \sin \theta$. The second appears more complex, but if we differentiate it we find

$$
\frac{d}{d t}\left(2 \cot t_{2}^{1} \theta \log \sec _{2}^{1} \theta\right)=1-\operatorname{cosec}_{2}^{2} \frac{1}{2} \theta \log \sec \frac{1}{2}_{2} \theta
$$

and if we divide it by $\sin \theta$ we find

$$
2 \cot \int_{2}^{1} \theta \log \sec \int_{2}^{1} \theta / \sin \theta=\operatorname{cosec}^{2} \log _{2}^{1} \theta \log \sec \int_{2}^{1} \theta \text {, }
$$

so that $2 \cot \int_{2} \theta \log \sec \int_{2}^{1} \theta$ is half that function which has the property that its radial scale error is equal and opposite to its perpendicular scale error. It is in fact half the function which solves the differential equation

$$
(\operatorname{ar} d \theta-\mathrm{I})+(r / \sin \theta-\mathrm{I})=0 \text {, }
$$

that is,

$$
r=4 \cot \frac{1}{2} \theta \log \sec \frac{1}{2} \theta+B \cot \frac{1}{2} \theta \text {, }
$$

where in the present case $B=0$. It is therefore to a first approximation half a function giving an Equal Area Projection, the characteristic function of which is $r=2 \sin \frac{1}{2} \theta$. This is seen more readily from the expansions of these two functions, viz.,

$$
\begin{aligned}
& 2 \sin 1 \theta=\theta-2_{21}^{1} \theta^{*}+\pi_{1}^{1} \overline{2} 0 \theta^{5}-\ldots, \\
& 4 \cot \frac{1}{2} \theta \log \sec \frac{1}{2} \theta=\theta-2_{4}^{1} \theta^{3}-\frac{1}{4 \times 0^{5}} \theta^{5}-\ldots
\end{aligned}
$$

We see therefore that Airy's Projection is in form practically a mean between an Orthomorphic and an Equal Area Projection, but the orthomorphic part must be multiplied by a constant $A$ which has the effect of reducing the positive scale error at the outer boundary, at the expense of introducing a negative scale error at the centre. The function

$$
r=4 \cot \prod_{2}^{1} \theta \log \sec \operatorname{lag}_{2}^{1} \theta
$$

does not seem to have been used by itself for a nap projection, nor does there seem to be any reason for preferring it to the simpler and practically identical Épual Irea function $r=2 \sin { }_{2}^{1} \theta$. It lends itself more readily, however, to the mathematical operations necessary to determine the constants of the Minimum Error P'rojection and is a useful guide when dealing with the absolute Fopual Arat function in both the \%enithal and Conical Clatises.

Liy substituting the value of $r$ as found above in the expression for $M$ and integrating, we can find the value of $M$ and hence of $\mu$ in terms of $\beta$. IIt shall finel

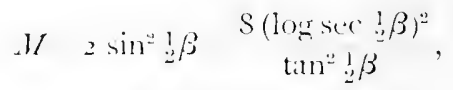

$$
\begin{aligned}
& \mu^{2} \quad J(1-\cos \beta) \quad 1-\frac{4\left(\log \sec \frac{1}{2} \beta\right)^{2} \sec ! \beta}{\sin ^{3} ! \beta} .
\end{aligned}
$$


Clarke in his Paper quoted above does not appear to have taken this step, but he evaluates the value of $M$ for the particular case of $\beta=\operatorname{II} 3^{\circ} 30^{\prime}$ by mechanical integration, getting the value $M=0.1569$. 'The above formula gives $0^{\circ}$ 5 588 , which checks Clarke's value exactly.

\section{Airy's Solution expanded.}

It is instructive and more useful for the purpose of comparison with other solutions to expand Airy's solution as follows :

We have

$$
r=A \tan \frac{\mathrm{I}}{2} \theta+2 \cot \frac{\mathrm{I}}{2} \theta \log \sec \frac{\mathrm{I}}{2} \theta .
$$

Now

$$
\tan \frac{\mathrm{I}}{2} \theta=\frac{\mathrm{I}}{2} \theta+\frac{\mathrm{I}}{24} \theta^{3}+\frac{\mathrm{I}}{240} \theta^{5}+\frac{\mathrm{I} 7}{40320} \theta^{7}+\ldots,
$$

$$
2 \cot \frac{\mathrm{I}}{2} \theta \log \sec \frac{\mathrm{I}}{2} \theta=\frac{\mathrm{I}}{2} \theta-\frac{\mathrm{I}}{48} \theta^{3}-{ }_{960}^{\mathrm{I}} \theta^{5}-\frac{\mathrm{I}}{\mathrm{I} 7920} \theta^{i}-\ldots,
$$

$$
\sin \theta=\theta-\frac{\mathrm{I}}{6} \theta^{3}+\frac{\mathrm{I}}{\mathrm{I} 20} \theta^{5}-\frac{\mathrm{I}}{50.40} \theta^{7}+\ldots .
$$

We know that the first term of the constant $A$ expanded in terms of $\beta$ is $\mathbf{r}$, and we shall save some trouble by writing $A=\mathrm{I}+b$, where $b$ only is to be found. We can then add the first two expansions together and write

thus

$$
r=\theta+\frac{\mathrm{I}}{48} \theta^{3}+\frac{\mathrm{I}}{320} \theta^{5}+\frac{59}{\mathrm{I} 6 \mathrm{I}, 280} \theta^{7}+\ldots+\frac{\mathrm{I}}{2} b \theta\left(\mathrm{I}+\frac{\mathrm{I}}{\mathrm{I} 2} \theta^{2}+\frac{\mathrm{I}}{\mathrm{I} 20} \theta^{4} \ldots\right),
$$

$$
\begin{aligned}
d r / d \theta & =\mathrm{I}+\frac{\mathrm{I}}{\mathrm{I} 6} \theta^{2}+\frac{\mathrm{I}}{64} \theta^{4}+\frac{59}{23040} \theta^{6}+\ldots+\frac{\mathrm{I}}{2} b\left(\mathrm{I}+\frac{\mathrm{I}}{4} \theta^{2}+\frac{\mathrm{I}}{24} \theta^{4} \ldots\right), \\
r / \sin \theta & =\mathrm{I}+\frac{3}{\mathrm{I} 6} \theta^{2}+\frac{5}{\mathrm{I} 9^{2}} \theta^{4}+\frac{77}{23040} \theta^{6}+\frac{\mathrm{I}}{2} b\left(\mathrm{I}+\frac{\mathrm{I}}{4} \theta^{2}+\frac{\mathrm{I}}{24} \theta^{4}\right) ;
\end{aligned}
$$

therefore

$$
\begin{aligned}
& M=\int_{0}^{\beta}\left\{(d r / d \theta-\mathrm{I})^{2}+(r / \sin \theta-\mathrm{I})^{2}\right\} \sin \theta d \theta \\
& =\int_{0}^{\beta}\left\{\frac{\mathrm{I}}{25^{6}} \theta^{4}\left(\mathrm{I}+\frac{\mathrm{I}}{4} \theta^{2}+\frac{59}{\mathrm{I} 440} \theta^{4}\right)^{2}\right. \\
& +\frac{b \theta^{2}}{16}\left(\mathrm{I}+\frac{\mathrm{I}}{4} \theta^{2}+\frac{59}{\mathrm{I} 440} \theta^{4}\right)\left(\mathrm{I}+\frac{\mathrm{I}}{4} \theta^{2}+\frac{\mathrm{I}}{24} \theta^{4}\right)+\frac{i^{2}}{4}\left(\mathrm{I}+\frac{\mathrm{I}}{4} \theta^{2}+\frac{\mathrm{I}}{24} \theta^{4}\right)^{2} \\
& +\frac{\mathrm{I}}{256} \theta^{4}\left(3+\frac{5}{\mathrm{I} 2} \theta^{2}+\frac{77}{\mathrm{I} 440} \theta^{4}\right)^{2}+\frac{b \theta^{2}}{\mathrm{I} 6}\left(3+\frac{5}{\mathrm{I} 2} \theta^{2}+\frac{77}{\mathrm{I} 440} \theta^{4}\right)\left(\mathrm{I}+\frac{\mathrm{I}}{4} \theta^{2}+\frac{\mathrm{I}}{24} \theta^{4}\right) \\
& \left.+\frac{b^{2}}{4}\left(\mathrm{I}+\frac{\mathrm{I}}{4} \theta^{2}+\frac{\mathrm{I}}{24} \theta^{\grave{2}}\right)^{2}\right\} \sin \theta d \theta \\
& =\int_{0}^{\beta}\left\{\frac{\theta^{5}}{25^{6}}\left(\mathrm{10}+3 \theta^{2}+\frac{23}{36} 6^{4}\right)+\frac{b \theta^{3}}{\mathrm{I} 6}\left(4+\frac{5}{3} \theta^{2}+\frac{77}{150} \theta^{4}\right)\right. \\
& \left.+\frac{b^{2} \theta}{4}\left(2+\theta^{2}+\frac{7}{24} \theta^{4}\right)\right\}\left(\mathrm{I}-\frac{\mathrm{I}}{6} \theta^{2}+\frac{\mathrm{I}}{\mathrm{I} 20} \theta^{4}\right) d \theta \\
& =\int_{0}^{\beta}\left\{\frac{\theta^{5}}{256}\left(10+\frac{4 \theta^{2}}{3}+\frac{2 \theta^{4}}{9}\right)+\frac{b \theta^{3}}{16}\left(4+\theta^{2}+\frac{\mathrm{II} \theta^{4}}{60}\right)+\frac{b^{2} \theta}{4}\left(2+\frac{2 \theta^{2}}{3}+\frac{17 \theta^{4}}{120}\right)\right\} d \theta \\
& =\frac{\beta^{6}}{25^{6}}\left(\frac{5}{3}+\frac{\beta^{2}}{6}+\frac{\beta^{4}}{45}\right)+\frac{b \beta^{4}}{16}\left(\mathrm{I}+\frac{\beta^{2}}{6}+\frac{\mathrm{II} \beta^{4}}{480}\right)+\frac{b^{2} \beta^{2}}{4}\left(\mathrm{I}+\frac{\beta^{2}}{6}+\frac{\mathrm{I} 7 \beta^{4}}{720}\right) \text {. }
\end{aligned}
$$


Putting the differential coefficient of this with respect to $b$ equal to zero we find for the value of $b$ to make this expression a minimum

and consequently

$$
\begin{gathered}
b=-\frac{\beta^{2}}{8}+\begin{array}{c}
\beta^{6} \\
\text { II } 520 \\
A-\mathrm{I}-\frac{\beta^{2}}{8}+\beta^{6} \\
11520
\end{array},
\end{gathered}
$$

which will be found to agree with the expansion of $2 \cot ^{2} \frac{1}{2} \beta \log \sec \frac{1}{2} \beta$ as found above.

Substituting this value of $b$ in the expression for $M$ we find

$$
\begin{aligned}
& \mu=\frac{5 \beta^{i}}{768}+\frac{\beta^{4}}{1536}+\frac{\beta^{\prime \prime \prime}}{11520} \ldots \\
& -\frac{\beta^{6}}{256}-\frac{\beta^{2}}{153^{6}}-\frac{\beta^{11}}{11520} \ldots \\
& ={ }_{3} s^{6}+{ }^{6} \text { to } 3 \text { terms expanded, }
\end{aligned}
$$

dividing $1 /$ by $1-\cos \beta={ }_{2}^{\beta^{2}}\left(1-\frac{\beta^{2}}{12}+\ldots\right)$ we find $\mu^{2}$ and consequently $\mu=\operatorname{li}_{192}^{\beta^{2}}\left(1+\frac{\beta^{2}}{24} \ldots\right)={ }_{14}^{\beta^{2}}$ nearly. If we substitute the value found for $b$ in the original expansions we find for the scale values at the centre, where $\theta=0,(d r d \theta)_{0}=(r \sin \theta)_{0}=1+{ }_{2}^{1} l=1-\frac{\beta^{2}}{16}+\ldots ;$ and for the limiting radius and scale values thereat

$$
\begin{aligned}
& r_{\beta}=\beta\left(1-\frac{\beta^{2}}{2+}-\frac{\beta^{4}}{480}-\frac{\beta^{6}}{8960} \ldots\right) . \\
& (d r d \theta)_{\beta}=1, \\
& (r \sin \theta)_{\beta}=1+\frac{\beta^{2}}{s}+\frac{\beta^{4}}{y_{6}^{6}}+\ldots
\end{aligned}
$$

Supposing instead of using the value of the constant $A$ which makes the mean scpuare error a minimum, we had used $A=1$, as did Airy, then we shall fiod the values of the corresponding quantities by making $b-0$; in our formulac: thus $(d r d \theta)_{11} \quad(r \sin \theta)_{11}=1$,

$$
\begin{aligned}
& r_{\beta} \beta\left(1, \begin{array}{ll}
\beta^{2} & \beta^{4} \\
4 & 320
\end{array}\right) \text {, }
\end{aligned}
$$

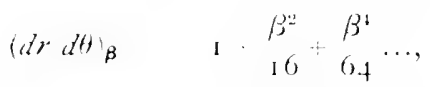

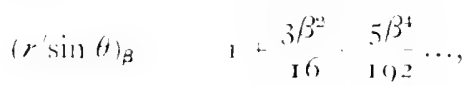

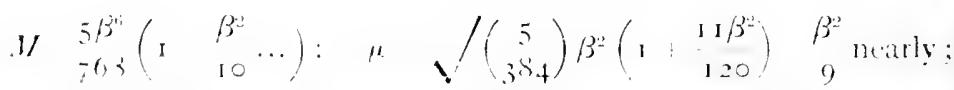

6) that boh the masmum and mean sahe errors are about 50 per cent. higher. liry solution with 1 a does not enen give the minimum cror 
solution with the stipulation that the scales shall be true at the centre, as will be proved later, which shews the importance of correctly evaluating the constant $A$.

To find for what value of $\theta$ in the Minimum Error solution the perpendicular scale error vanishes put $r / \sin \theta=0$ and we find $\frac{36^{2}}{16} \cdots-\frac{\beta^{2}}{16}=0$ or $\theta=\frac{\mathrm{I}}{\sqrt{ } 3} \beta$ nearly.

It might appear that the expansion for $M$ contains no terms beyond $\beta^{i}$; there is, however, a term in $\beta^{12}$ as follows :

$$
M=\frac{\beta^{6}}{3^{8} 4}\left(1-\frac{\beta^{6}}{3^{02}+0} \ldots\right) \text {. }
$$

\section{Breusing's Projection.}

As we have already remarked, Breusing conceived a projection which is the geometrical mean between the Orthomorphic and Equal Area, viz., $r=2 \sqrt{ } /\left(\sin \frac{1}{2} \theta \tan \frac{1}{2} \theta\right)$, and Hammer has pointed out how nearly this agrees with Airy's solution taking $A=\mathrm{I}$. The writer finds that whether the arithmetic, geometric or harmonic mean of these two functions be taken, we obtain a projection agreeing with Airy's to 2 terms in the expansion of $M$, and to at least that order in the other quantities. The harmonic mean leads to a much simpler function than either of the others and we shall investigate it accordingly.

\section{The Breusing (Harmonic) Minimum Error Projection.}

The harmonic mean of $2 \tan \frac{1}{2} \theta$ and $2 \sin \frac{1}{2} \theta$ is $4 \tan \frac{1}{4} \theta$. Consequently write $r=4 A \tan l_{t} \theta$ and determine the best value of $A$ as before. We shall find

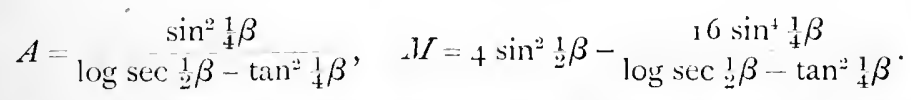

To shew how nearly this projection agrees with Airy's we shall find in applying it to the extreme case taken by Clarke, viz., $\beta=113^{\circ} 30^{\prime}$, the limiting radius comes out as $1{ }^{\circ} 578 \mathrm{I}$ as against ${ }^{\circ} 5760$, and the value of $M$ as $0^{1} 1579$ as against $0^{1} 1_{5} 69$, i.e. its average scale error is only 1 part in 3000 greater.

By expanding it in the same way as Airy's we shall find

$$
M=\frac{\beta^{6}}{25^{6}}\left(\begin{array}{c}
\frac{5}{3}+\frac{\beta^{2}}{12}+\frac{49 \beta^{4}}{5760} \cdots \\
\left.-1-\frac{\beta^{2}}{12}-\frac{\beta^{4}}{120} \ldots\right)=\frac{\beta^{46}}{3^{84}}\left(1+\begin{array}{c}
\beta^{4} \\
3_{4}^{8}
\end{array}\right) .
\end{array}\right.
$$

For the scale values at the centre we get

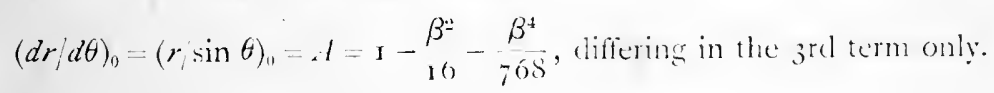


At the limiting radius we find

$$
\begin{aligned}
& r_{\beta}=\beta\left(1-\frac{\beta^{2}}{24}-\frac{\beta^{4}}{480}-\frac{\beta^{6}}{8960} \ldots\right) \text {, agreeing to } 4 \text { terms with Airy's, } \\
& (d r d \theta)_{\beta} \quad \text { I } \quad-\frac{\beta^{4}}{3^{8}+} \cdots \quad, \quad, \quad 2 \quad, \quad \text {, } \\
& (r \sin \theta)_{\beta}=\quad 1+\frac{\beta^{2}}{8}+\frac{\beta^{4}}{96} \cdots \cdots \quad, \quad, \quad, \quad 3 \quad, \quad,
\end{aligned}
$$

This much simpler formula thus leads to almost identically the same result as diry's, and when we remember that in the extreme case of $\beta 113^{\circ} 30^{\prime}$, a map covering more than two-thirds the surface of the globe, the maximum error is actually $72 \%$, it certainly does not seem worth while using the more complex formula of Airy to reduce the mean error by only I/3000th part.

\section{The Orthomorphic Minimum Error Projection.}

In this we have $r=2 . A$ tan $?_{2}^{1} \theta$ and we shall find that $A$ has exactly the same value as in Airy's and $/ /$ double that value, viz.,

$$
\begin{aligned}
& A-2 \cot ^{2}{ }_{2}^{1} \beta \log \sec \int_{2}^{1} \beta=1-\frac{\beta^{2}}{S^{2}}+\beta_{\text {I } 520} \cdots, \\
& M=4 \sin _{2}^{2} \beta-\frac{16\left(\log \sec { }_{2}^{1} \beta\right)^{2}}{\tan _{2}^{2} \beta}=\frac{\beta^{6}}{192} ; \mu \quad \frac{\beta^{2}}{\sqrt{96}}\left(1+\frac{\beta^{2}}{24}\right) .
\end{aligned}
$$

The scale value at the centre is

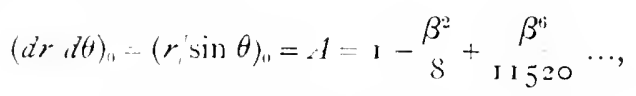

and the limiting radius and scale value thereat have the same values as in Airy's.

\section{The Equal Area Minimum Error Projection.}

In this we have $r-2.4 \sin \frac{1}{2} \theta$ and on integrating we shall find

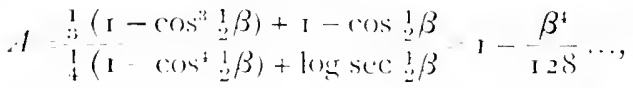

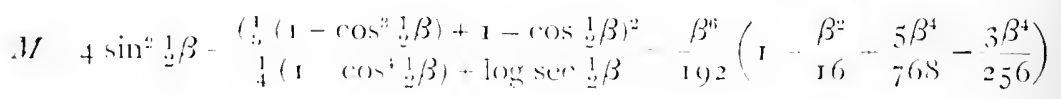

$$
\begin{aligned}
& 192\left(1-\frac{\beta^{3}}{16}-\frac{\beta^{*}}{102}\right),
\end{aligned}
$$

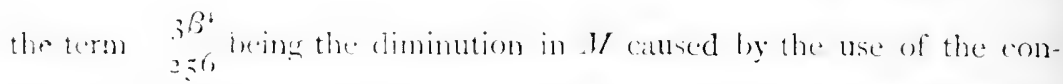
siont 4 . This syotem is therefore caprable of pratically no improvement by the use of a wale constant. 
The scale values at the centre are $A=\mathbf{I}-\frac{\beta^{4}}{128} \ldots$, and at the outer limit of the map we have

$$
\begin{aligned}
r_{\beta} & =\beta\left(\mathrm{I}-\frac{\beta^{2}}{24}-\frac{7 \beta^{4}}{960}\right), \\
(d r / d \theta)_{\beta} & =\mathrm{I}-\frac{\beta^{2}}{8}-\frac{\beta^{4}}{192} \cdots, \\
(r / \sin \theta)_{\beta} & =\mathrm{I}+\frac{\beta^{2}}{S}+\frac{\beta^{4}}{192} \cdots
\end{aligned}
$$

The use of a scale constant with $2 \sin \frac{1}{2} \theta$ is in fact not appropriate to this Projection, for, as we see, it destroys the characteristic property of reciprocity of scale values, at any rate after the first 2 terms. 'The same remark applies to its prototype $r=4 \cot \frac{1}{2} \theta \log \sec \operatorname{li}_{2}^{1} \theta$ for which also $M=\frac{\beta^{3}}{192}$. It will be shewn later that for this we should write the full formula $r=4 \cot \frac{1}{2} \theta \log \sec \frac{1}{2} \theta+2 B \cot \frac{1}{2} A$, and after determining $B$ so as to make $M$ a minimum for a zone from $\theta=\alpha$ to $\theta=\beta$, we put $\alpha=0$, which amounts to making $B=0$ originally.

\section{The Equidistant Minimum Error Projection.}

Clarke in his Paper on Airy's Projection published in the Philosophical Magazine for April 1862 compares it with two others. One, the Perspective Projection of James, has received considerable notice but the other, the Equidistant, or Equal Radial as Clarke calls it, is seldom mentioned, although as he shews when drawn with the same limiting radius as Airy's its $M /$ is to Airy's as $104^{\circ} 7: 100^{\circ}$. Clarke does not explain very clearly why he draws the limiting radius equal to Airy's, but what he does has the effect practically of writing the equation of the Equidistant as $r=A \theta$ and then of determining $A$ so that the mean square scale error shall be a minimum.

On applying our method we find

$$
\begin{aligned}
& A=\frac{\mathrm{I}-\cos \beta+\frac{1}{2} \beta^{2}}{\mathrm{I}-\cos \beta+\int_{0}^{\beta} \theta^{2} \operatorname{cosec} \theta d \theta}=\mathrm{I}-\frac{\beta^{2}}{2+}-\frac{7 \beta^{4}}{2 \mathrm{I} 60}-\frac{23 \beta^{5}}{3^{6} 2,880} \cdots,
\end{aligned}
$$

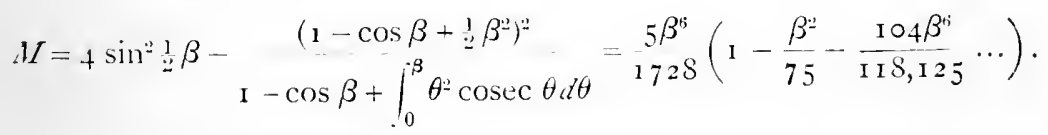

For $\beta=113^{\circ} 30^{\prime}$ this gives $M=0.163+$ as against $0^{\circ} 16+3$ found by Clarke by mechanical integration, for the case $r_{\beta}$ the same as in Airy's solution. The scale values at the centre of the map are

$$
A=\mathrm{I}-\frac{\beta^{2}}{2+}-\frac{7 \beta^{4}}{2160} \cdots
$$


and at the outer boundary we find

$$
\begin{aligned}
r_{\beta}=\beta\left(1-\frac{\beta^{2}}{24}-\frac{7 \beta^{4}}{2160} \ldots\right), \text { agreeing with Airy's to } 2 \text { terms, } \\
(d r / d \theta)_{\beta}=1-\frac{\beta^{2}}{24}-\frac{7 \beta^{4}}{2160} \cdots \\
(r / \sin \theta)_{\beta} \quad 1+\frac{\beta^{2}}{S}+\frac{\beta^{4}}{108} \cdots \quad, \quad, \quad, \quad, \quad, \quad, \quad \text { I term, }
\end{aligned}
$$

The value of the mean square error $\mu$

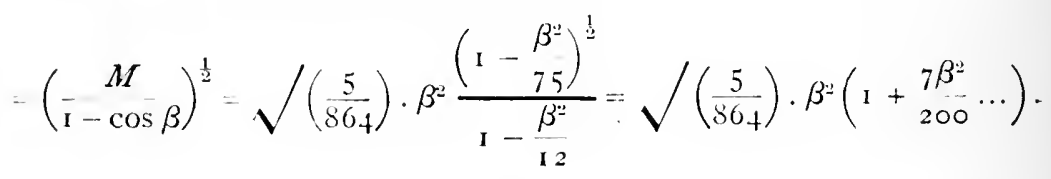

This exceeds the value in Airy's in the ratio $\sqrt{192}: \sqrt{1} 72 \cdot 8=105: 100$. In the writer's opinion this slight increase in the value of the average scale error is far out-weighed by the valuable property of uniform radial scale and the simplicity of the formula of the Equidistant Zenithal Projection. If we make $A=\mathrm{I}$ we shall find $M=\frac{\beta^{6}}{2 \mathrm{I} 6}$ as against $\frac{5 \beta^{6}}{768}=\frac{\beta^{6}}{153 \cdot 6}$ in Airy's formula. Therefore Airy's Projection as exhibited by the author and other writers is not per se so good as the Equidistant in the ratio

$$
\sqrt{216}: \sqrt{1} 53 \cdot 6=119: 100 .
$$

The merits of this projection in comparison with others are described in a Paper by General de Coatpont in the bulletin de la Société de Géosraphic for $1 \$_{94}$, but without the use of the scale constant. Its invention is attributed to Guillaume Postel in $158 \mathrm{r}$.

\section{Modification of Minimum Error Projections by making total area of map true.}

It will be observed that in all the Minimum Error Projections considered the formulac for the limiting radius $r_{\beta}$ when expanded agree to the first two terms, vi\%, $r_{\beta}=\beta\left(1-\frac{\beta^{2}}{2+} \ldots\right)$. Now in the lipual Area l'rojection $r_{\beta} \beta\left(\mathrm{I}-\frac{\beta^{2}}{24}+\frac{\beta^{4}}{1020} \ldots\right)$. It therefore appears that we shall sacrifiet very litte in the value of the average scalle error if we choose our scale constant so that equalizy of total arca is absolutely ensured, and this is a valuable (quality in any map).

In some cases, ats we shall shew, the formula for the quantity $A$ is simplified. In every ane ensiclered the expansion for $M$ is altered only

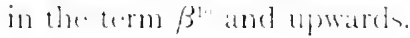




\section{(i) Airy's Projection with total area true.}

Here

$$
r_{\beta}=A \tan \frac{1}{2} \beta+2 \cot \frac{1}{2} \beta \log \sec \frac{1}{2} \beta ;
$$

therefore to make total area true equate this to $2 \sin \frac{1}{2} \beta$, whence

$$
A=2 \cos \frac{1}{2} \beta-2 \cot ^{2} \frac{1}{2} \beta \log \sec \frac{1}{2} \beta=\mathbf{I}-\frac{\beta^{2}}{8}+\frac{\beta^{4}}{\mathrm{I}^{2}}-\frac{\beta^{6}}{7680} \cdots
$$

Substituting this we find

$$
\begin{aligned}
M & =\frac{\beta^{6}}{384}\left(\mathrm{I}+\frac{\beta^{4}}{3^{84}}\right) ; \mu=\frac{\beta^{2}}{\mathrm{II} 9^{2}}\left(\mathrm{I}+\frac{\beta^{*}}{24} \ldots\right), \\
r_{\beta} & =\beta\left(\mathrm{I}-\frac{\beta^{2}}{24}+\frac{\beta^{4}}{\mathrm{I} 920}\right)=2 \sin \frac{1}{2} \beta, \\
(d r / d \theta)_{\beta} & =\mathrm{I}+\frac{\beta^{4}}{384} ; \quad(r / \sin \theta)_{\beta}=\mathrm{I}+\frac{\beta^{2}}{8}+\frac{5 \beta^{4}}{3^{8} 4}+\ldots=\sec \operatorname{l}_{2}^{1} \beta .
\end{aligned}
$$

(ii) Breusing (Harmonic) Projection with total area true.

Here

$$
r_{\beta}={ }_{4} A \tan \frac{1}{4} \beta=2 \sin \frac{1}{2} \beta=4 \sin \frac{1}{4} \beta \cos \frac{1}{4} \beta,
$$

whence

$$
A=\cos ^{2}{ }_{4}^{1} \beta=\mathrm{I}-\frac{\beta^{2}}{16}+\frac{\beta^{4}}{765}-\frac{\beta^{6}}{92160}+\ldots \text {, }
$$

and

$$
\begin{aligned}
& M=\frac{\beta^{6}}{3^{84}}\left(\mathrm{I}+\frac{\mathrm{II} \beta^{4}}{3^{8}+0}\right) ; \quad \mu=\frac{\beta^{2}}{\sqrt{1} 9^{2}}\left(\mathrm{I}+\frac{\beta^{2}}{24} \ldots\right), \\
& r_{\beta}=\beta\left(\mathrm{I}-\frac{\beta^{2}}{24}+\frac{\beta^{4}}{1920} \ldots\right)=2 \sin \frac{1}{2} \beta,
\end{aligned}
$$

$$
(d \boldsymbol{r} / d \theta)_{\beta}=\mathrm{I} ; \quad(\boldsymbol{r} / \sin \theta)_{\beta}=\mathrm{I}+\frac{\beta^{2}}{8}+\frac{5 \beta^{4}}{38+} \ldots=\sec \frac{1}{2} \beta .
$$

(iii) Orthomorphic Projection with total area true.

Here

$$
r_{\beta}=2 A \tan \frac{1}{2} \beta=2 \sin \frac{1}{2} \beta,
$$

therefore

$$
A=\cos \frac{1}{2} \beta=\mathrm{I}-\frac{\beta^{2}}{8}+\frac{\beta^{4}}{384}-\frac{\beta^{6}}{46080}+\ldots,
$$

whence

$$
M=\frac{\beta^{6}}{19^{2}}\left(1+\frac{\beta^{4}}{768}\right) ; \mu=\frac{\beta^{2}}{\sqrt{96}}\left(1+\frac{\beta^{2}}{24} \cdots\right),
$$

$$
(d r / d \theta)_{\beta}=(r / \sin \theta)_{\beta}=\mathrm{I}+\frac{\beta^{2}}{8}+\frac{5 \beta^{4}}{384} \ldots=\sec \stackrel{1}{2} \beta .
$$

(iv) 'The Equal Area Projection of course remains unchanged and we have

$$
\begin{aligned}
r_{\beta} & =2 \sin \frac{1}{2} \beta, A-\mathrm{I}, \\
M & =\frac{\beta^{6}}{\mathrm{I} 9^{2}}\left(\mathrm{I}-\frac{\beta^{2}}{\mathrm{I} 6}+\frac{5 \beta^{4}}{768}\right): \mu=\frac{\beta^{2}}{9^{6}}\left(\mathrm{I}-\frac{\beta^{2}}{48} \ldots\right), \\
(d r / d \theta)_{\beta} & =\mathrm{I}-\frac{\beta^{2}}{8}+\frac{\beta^{4}}{3^{8}} \ldots \cos \frac{1}{2} \beta, \\
\left(r_{1} / \sin \theta\right)_{\beta} & =\mathrm{I}+\frac{\beta^{2}}{8}+\frac{5 \beta^{4}}{3^{8}} \ldots=\sec _{2}^{1} \beta .
\end{aligned}
$$




\section{(v) Equidistant Projection with total area true.}

Here

$$
r_{\beta}=A \beta=2 \sin \frac{1}{2} \beta,
$$

whence $A=\frac{2}{\beta} \sin \frac{1}{2} \beta=\mathrm{I}-\frac{\beta^{2}}{24}+\frac{\beta^{4}}{1920}-\ldots$,

$$
\begin{aligned}
M & =\frac{5 \beta^{6}}{1728}\left(\mathrm{I}-\frac{\beta^{2}}{75}+\frac{40,4 \mathrm{I} 7 \beta^{4}}{\mathrm{1} 0,080,000}\right) ; \mu=\sqrt{\left(\frac{5}{864}\right) \beta^{2}\left(\mathrm{I}+\frac{7 \beta^{2}}{200}\right) .} \\
(d r / d \theta)_{\beta} & =\mathrm{I}-\frac{\beta^{2}}{24}+\frac{\beta^{4}}{1920}-\ldots={ }_{\beta}^{2} \sin \frac{1}{2} \beta, \\
(r / \sin \theta)_{\beta} & =\mathrm{I}+\frac{\beta^{2}}{8}+\frac{5 \beta^{4}}{38_{4}}+\ldots=\sec \frac{1}{2} \beta .
\end{aligned}
$$

The perpendieular scale crror vanishes when $\theta=1_{2}^{1} \beta$ nearly.

\section{The Minimum Error Perspective Projection.}

The geometrical meaning of the Perspective Projection is as follows: Let the circle in Figg. I with centre $O$ represent a section through the

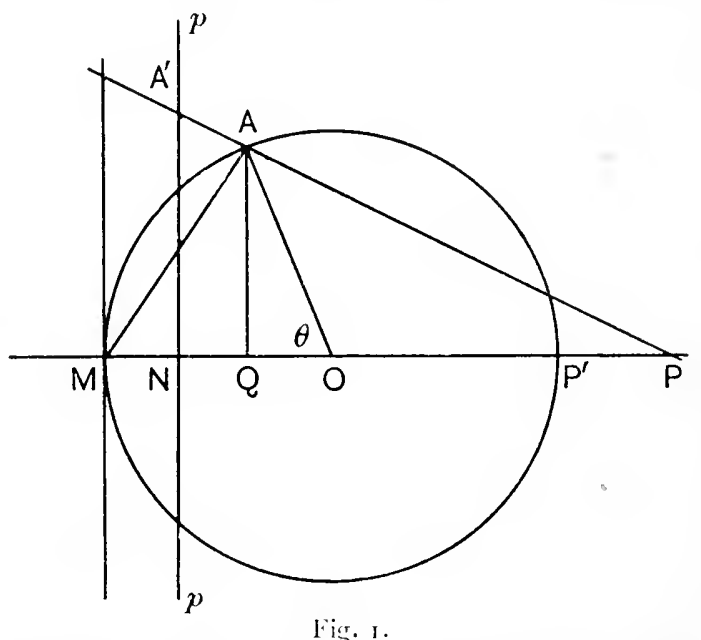

centre of the Earth, and draw a line through $O$ and a point $M$ on the surface which corresponds with the centre of the map. Then choose a point $P$ ' on the dianeter $M I O$, prolonged if necessary, from which points on the surfice of the Warth caun be projected by straight lines on to a plane of projection th which is at right angles to the line MOP. Let $A$ be such a print and $A^{\prime}$ its projection, then the distance $N A^{\prime}$ is $r$ in our formula, and the angle $V O A$ is $\theta$; denote the distance $O P$ by $h$ and the distance $N P^{\prime}$ ly k. The raclius of the Earth $O A$ is taken as unity. I)raw $A Q$ perpendicular to.$/ O P P^{\prime}$ and let $N$ be the point where the line $M O P^{\prime}$ cuts the plane the Then

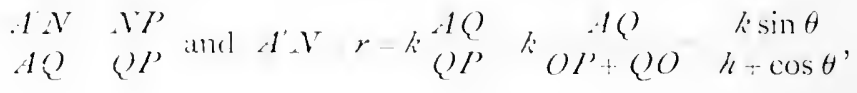


which is the characteristic formula of this class. It should be noted that both Germain and Thomas Craig make the mistake of saying that $k$ is the distance of the plane of projection from the centre, or rather Craig says, "... making $h$ the distance of the eye from the centre and $k$ the distance of the plane of projection from the same point...," whereas $k$ is the distance of the plane of projection from the eye (i.e. from point $P$ ).

If the point $P$ is taken at $P^{\prime}$ on the surface of the Earth and the plane $p p$ tangent at $M$ on the opposite surface, then $h=1$ and $k=\mathbf{2}$, so that

$$
r=\frac{2 \sin \theta}{1+\cos \theta}=2 \tan \frac{1}{2} \theta
$$

and we get the ordinary Orthomorphic or Stereographic Projection.

If we take $h=0$ and $k=\mathrm{I}$ we get $r=\tan \theta$ the Gnomonic Projection.

If we take $k=\mathrm{I}+h$ so that the plane of projection becomes tangent at $M$, and then make $h$ increase without limit so that the projecting rays become parallel, we get $r=\sin \theta$, the Orthographic Projection.

Several Perspective Projections have been put forward from time to time with the plane of projection tangent at $l K$, or taking $k=\mathbf{1}+h$, with values of $h$ ranging from 1 to $2.73, e . g$. those of Parent, Lowry, La Hire, etc.

General Sir H. James, Director General of the Ordnance Survey, in seeking for the best values of $h$ and $k$ for a map covering two-thirds of the sphere, or $\beta=113^{\circ} 30^{\prime}$, either by intuition or luck hit upon the value $h=\mathrm{I} \cdot 5$ and he took the plane $p p$ at the same distance from the centre of the Earth as the plane of the tropics, that is, he made

$$
N O=\sin 23^{\circ} 30^{\circ}=0.399 \cdots,
$$

and therefore $k=N O+O P=\mathrm{I}_{5} \cdot 5+0.399=1 \cdot S 99 \ldots$

Fiorini shews (pp. $213^{-4}$ ) that with these values of $h$ and $k$ James' Projection gives

$$
r_{\beta}=1.58 \mathrm{I} 2, \quad M=0.1720, \quad(d r d \theta)_{\beta} \quad 0.6292, \quad(r \sin \theta)_{\beta}=\mathrm{I} \cdot 72+3 .
$$

Clarke, by taking $k$ so that $r_{\beta}=1 \cdot 5760$, the same as in Airy's Projection, fincls $M=0.1718$, which differs very little from the value with James' values of $h$ and $k$; but on finding by integration the values of $h$ and $k$ which make $M$ a minimum Clarke obtains $h=1 \cdot 36763, k=1 \cdot 6626 \mathrm{I}, r_{\beta}=1.5737$, $\boldsymbol{M}=0 . \mathrm{I} 626,(d r / d \theta)_{\beta}=0.8053,(r / \sin \theta)_{\beta}=\mathrm{I} \cdot 7 \mathrm{I} 60$.

Clarke on integrating the value of $I /$ brings it into the form

$$
M=+\sin ^{2} \beta-\frac{B^{2}}{A},
$$

where

$$
\begin{aligned}
& A=\left\{\left(h^{2}+\mathrm{I}\right)\left(\frac{\mathrm{I}}{n}-\frac{\mathrm{I}}{m}\right)-h\left(h^{2}-\mathrm{I}\right)\left(\begin{array}{c}
\mathrm{I} \\
h^{2}
\end{array}-\frac{\mathrm{I}}{m^{2}}\right)+\left(\begin{array}{c}
\left.h^{2}-\mathrm{I}\right)^{2} \\
3
\end{array}\left(\begin{array}{cc}
\mathrm{I} & \mathrm{I} \\
h^{3} & m^{3}
\end{array}\right)\right\},\right. \\
& B=\left\{(h+1) \log \frac{m}{n}-\left(h^{2}-\mathrm{I}\right)\left(\frac{\mathrm{I}}{n}-\frac{\mathrm{I}}{m}\right)\right\},
\end{aligned}
$$

and where

$$
m=h+\mathrm{I}, \quad n=h+\cos \beta
$$


In the article on Mathematical Geography in the Encyclopaedia Britannica (Ninth Edition) Clarke puts these expressions into a more concise form, but the writer has found the above expressions easier to compute with a table of Squares, Cubes and Reciprocals like Barlow's. The differential coefficient of the above expression with respect to $h$ is unmanageable, and Clarke then proceeds to find by interpolation the value of $h$ which makes $B^{2} / A$ a maximum. The writer in checking the calculated values of $\log B^{2} / A$ in Clarke's example discovered a slight error in the value for $h=\mathrm{I} \cdot 39$ which should be 0.420712 instead of 0.420665 . This correction leads to uniformity in the second differences and gives $h=1 \cdot 368$ for the value of $h$ corresponding with the maximum.

\section{Perspective Projection expanded.}

By expanding the formula $r=\begin{gathered}k \sin \theta \\ h+\cos \theta\end{gathered}$ from the outset the writer has succeeded in finding expressions from which to compute the best values of $h$ and $k$ directly, as follows:

Write $k=(h+\mathbf{I})(\mathbf{I}-b)$, so that $b$ represents the distance from plane tangent at point $M$ to actual plane of projection $h p$. Also write $c=\frac{\mathrm{I}}{h+\mathrm{I}}$ and expand $\cos \theta$. Then we shall find

$$
\begin{aligned}
r & =(\mathrm{I}+b) \sin \theta\left\{\mathrm{I}+\frac{\theta^{2}}{2} c+\frac{\theta^{4}}{4} c\left(c-\frac{\mathrm{I}}{6}\right)+\frac{\theta^{6}}{8} c\left(c^{2}-\frac{c}{3}+\frac{\mathrm{I}}{90}\right) \ldots\right\} \\
& =\theta\left\{\mathrm{I}+\frac{\theta^{2}}{2}\left(i-\frac{\mathrm{I}}{3}\right)+\frac{\theta^{4}}{4}\left(c-\frac{c}{2}+\frac{\mathrm{I}}{30}\right)+\frac{\theta^{4}}{8}\left(c^{3}-\frac{2 c^{2}}{3}+\frac{c}{10}-\frac{\mathrm{I}}{630}\right) \ldots\right\}(\mathrm{I}-b),
\end{aligned}
$$

whence

$$
\begin{aligned}
& d r^{\prime} d \theta \quad 1+{ }_{2}^{3 \theta^{\prime \prime}}\left(c-\frac{1}{3}\right)+\frac{5 \theta^{4}}{4}\left(c^{2}-\frac{c}{2}+\frac{1}{30}\right)+\frac{7 \theta^{4}}{8}\left(c^{3}-\frac{2 c^{2}}{3}+\frac{c}{10}-\frac{1}{630}\right) \cdots \\
& -b\left\{\mathrm{I}+\frac{3^{\theta^{2}}}{2}\left(c-\frac{\mathrm{I}}{3}\right)+\frac{5^{\theta^{4}}}{4}\left(c-\frac{c}{2}+\frac{\mathrm{I}}{30}\right)\right\} \text {, } \\
& r / \sin \theta \quad \mathrm{I}+\frac{\theta^{\prime \prime}}{2} c+\frac{\theta^{4}}{4} i\left(c-\frac{\mathrm{I}}{6}\right)+\frac{\theta^{4}}{8} c\left(c^{2}-\frac{c}{3}+\frac{\mathrm{I}}{90}\right) \ldots
\end{aligned}
$$$$
-b\left\{1+\frac{\theta^{2}}{2} c+\theta_{4}^{\theta^{4}} c\left(c-\frac{1}{6}\right) \cdots\right\} \text {. }
$$

Forming the quantity

$$
. \quad \int_{0}^{\beta}:(d r d \theta-\mathrm{r})^{2}+(r \sin \theta-\mathrm{r})^{2 !} ; \sin \theta d \theta
$$

in the usual way and integrating, we tinally arrive at

$$
\begin{aligned}
& .1 \beta_{2}\left(100^{2}-6 \cdot \cdots 1\right)+3 \beta^{2}\left(16 c^{*}-\frac{4 c^{2}}{3}+4 c-\frac{1}{3}\right) \\
& \left.3 \beta^{4}\left(\begin{array}{c}
35^{4} \\
2
\end{array}-\frac{59 c^{3}}{3}+\frac{5+5 c^{2}}{72}-\frac{9 c}{8}+\frac{7}{14+4}\right) \cdots\right\}
\end{aligned}
$$




$$
\begin{aligned}
& -b \frac{\beta^{4}}{4}\left\{4\left(c-\frac{\mathbf{I}}{4}\right)+\frac{\beta^{2}}{3}\left(\mathrm{I} 6 c^{2}-10 c+\frac{3}{2}\right)\right. \\
& \left.+\frac{\beta^{4}}{8}\left(40 c^{3}-\frac{107 c^{2}}{3}+\frac{146 c}{15}-\frac{23}{30}\right) \ldots\right\} \\
& +b^{2} \beta^{2}\left\{1+\frac{\beta^{2}}{2}\left(2 c-\frac{2}{3}\right)+\frac{\beta^{4}}{3}\left(\begin{array}{c}
11 c^{2} \\
4
\end{array}-\frac{7 c}{4}+\frac{31}{120}\right) \ldots\right\}
\end{aligned}
$$

Differentiating this with respect to $b$ we find the value of $b$ to make $M$ a minimum is

$$
b=\frac{\beta^{2}}{2}\left\{\left(c-\frac{\mathrm{I}}{4}\right)+\beta^{2}\left(\frac{c^{2}}{3}-\frac{c}{4}+\frac{\mathrm{I}}{24}\right)+\beta^{4}\left(\frac{\mathrm{I} 7 c^{2}}{288}-\frac{\mathrm{I} 9 c}{360}+\frac{\mathrm{I} \mathrm{I}}{960}\right) \ldots\right\}
$$

Substituting this we find

$$
\begin{aligned}
M= & \frac{\beta^{6}}{24}\left\{\left(10 c^{2}-6 c+\mathrm{I}\right)+\beta^{2}\left(\mathrm{I} 2 c^{3}-\frac{43^{2}}{4}+3 c-\frac{1}{4}\right)\right. \\
& \left.+\beta^{4}\left(\frac{21 c^{4}}{2}-\frac{59 c^{3}}{5}+\frac{109 c^{2}}{24}-\frac{27 c}{40}+\frac{7}{240}\right) \ldots\right\} \\
& -\frac{\beta^{6}}{24}\left\{\left(6 c^{2}-3 c+\frac{3}{8}\right)+\beta^{2}\left(10 c^{3}-9 c^{2}+\frac{21 c}{8}-\frac{\mathrm{I}}{4}\right)\right. \\
& \left.+\beta^{4}\left(\frac{61 c^{4}}{6}-\frac{285 c^{3}}{24}+\frac{197 c^{2}}{40}-\frac{407 c}{480}+\frac{1}{20}\right) \ldots\right\} \\
= & \frac{\beta^{6}}{24}\left\{\left(4 c^{2}-3 c+\frac{5}{8}\right)+\beta^{2}\left(2 c^{3}-\frac{7 c^{2}}{4}+\frac{3 c}{8}\right)\right. \\
& \left.+\beta^{4}\left(\frac{c^{4}}{3}+\frac{3 c^{3}}{40}-\frac{23 c^{2}}{60}+\frac{83 c}{480}-\frac{1}{48}\right) \cdots\right\}
\end{aligned}
$$

where the first two lines represent the value of $M$ when $b=0$, or the projection is made on the tangent plane as supposed by Tissot and many writers ; the second two lines represent the decrease in $M$ due to moving the plane to its best position $b$ units towards the centre of Earth; and the third two lines represent this best value of $M$. We can now find the best value of $c$ on either supposition, by differentiating the first or third lines with respect to $c$, and equating the result to zero.

Taking the general case first we get for a minimum

$$
8 c-3+\beta^{2}\left(6 c^{2}-\frac{7 c}{2}+\frac{3}{8}\right)+\beta^{4}\left(\frac{4 c^{3}}{3}+\frac{9 c^{2}}{40}-\frac{23 c}{60}+\frac{83}{480}\right)=0
$$

solving this by successive approximations we find

$$
c=\frac{3}{8}+\frac{3 \beta^{2}}{25^{6}}+\frac{7 \beta^{4}}{6 \mathrm{I}+40} \cdots=\frac{3}{8}\left(\mathrm{I}+\frac{\beta^{2}}{32}+\frac{7 \beta^{4}}{23040} \cdots\right) .
$$

Substituting this value of $c$ we find

$$
M=\frac{\beta^{4 i}}{3^{8}}\left(\mathrm{I}+\frac{5 \beta^{4}}{3072} \cdots\right)
$$


which differs from the same quantity in Airy's Projection by the term in $\beta^{10}$ only. We shall also find

$$
\begin{aligned}
& (d r / d \theta)_{0}=(r / \sin \theta)_{0}=\mathbf{I}-b \\
& =1-\frac{\beta^{2}}{16}-\frac{5 \beta^{4}}{153^{6}}-\frac{\beta^{6}}{2+576} \ldots \text {, agreeing with Airy's to } 2 \text { terms, } \\
& r_{\beta}=\beta\left(1-\frac{\beta^{2}}{24}-\frac{\beta^{4}}{450}-\frac{\beta^{6}}{8960} \cdots\right) \quad, \quad, \quad 4, \\
& (d r / d \theta)_{\beta}==1-\frac{5 \beta^{4}}{768} \cdots \\
& (r / \sin \theta)_{\beta}=1+\frac{\beta^{2}}{8}+\frac{\beta^{4}}{96} \cdots \\
& h=\frac{1}{i}-\mathrm{I}=\frac{5}{3}\left(\mathrm{I}-\frac{\beta^{2}}{20}+\frac{31 \beta^{4}}{28800} \cdots\right), \\
& k=(h+1)(1-b)=\frac{8}{3}\left(1-\frac{3 \beta^{2}}{32}-\frac{29 \beta^{4}}{46080} \cdots\right) .
\end{aligned}
$$

We shall now check these formulae by computing the values of $h, k$ and $M$ for the cases considered by Clarke and Fiorini, viz., $\beta=113^{\circ} 30^{\prime}$ and $\beta=90^{\circ}$. We find

$$
\begin{aligned}
& \beta=113^{\circ}, 30^{\circ} \\
& h=\mathrm{I} \cdot 367 \text { as against } \mathrm{I} \cdot 368 \\
& k=\mathrm{I} \cdot 660 \quad, \quad \mathrm{r} \cdot 66_{3} \\
& M=0.161_{3} \quad, \quad 0.1626 \\
& \begin{array}{lcl}
\multicolumn{4}{c}{\beta=y 0^{\circ}} \\
h=1.471 & \text { as against } & 1.470 \\
k=2.040 \quad, & 2.038 \\
M=0.0395 \text { I } & , & 0.03959
\end{array}
\end{aligned}
$$

The above expansions for $h$ and $k$ are therefore sufficiently exact to enable us to calculate the constants of the Minimum Error Perspective Projection for maps of the largest extent, and to calculate them directly instead of by the longer and more troublesome method hitherto employed.

\section{Perspective Projection on tangent plane.}

If the projection is made on the tangent plane we have

$$
\begin{aligned}
& M=\frac{\beta^{i}}{24}\left\{(10 \dot{c}-6 c+1)+\beta^{2}\left(12 c^{3}-\frac{43 c^{2}}{4}+3 c-\frac{1}{4}\right)\right.
\end{aligned}
$$

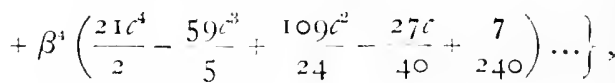

whence we shall find for the minimum

$$
\begin{aligned}
& \text { c }{ }_{10}^{3}\left(1+\frac{7 \beta^{2}}{200}+\frac{10 \beta^{4}}{120,000} \ldots\right) ; \quad / 1 \frac{\beta^{4 i}}{240}\left(1+\frac{13 \beta^{2}}{200} \cdots\right): \\
& h \quad{ }_{3}^{10}\left(\begin{array}{ll}
1 & 7 \beta^{2} \\
200
\end{array} \cdots\right)-1 \quad 7\left(1-\begin{array}{c}
\beta^{2} \\
20
\end{array} \cdots\right): \\
& k A h-1 \int_{3}^{10}\left(1-\frac{7 \beta^{2}}{200} \ldots\right) \text {. Also }(d r d \theta)_{1,}=(r \sin \theta)_{11} \quad \text { I : } \\
& r_{\beta} \beta\left(1-\frac{\beta^{\prime \prime}}{6} \cdots\right):(d r d \theta)_{\beta}-1-\frac{\beta^{\prime \prime}}{20} \cdots ;\left(r_{l} \sin \theta\right)_{\beta}=1+\frac{3 \beta^{\prime \prime}}{20} .
\end{aligned}
$$


These properties differ considerably from those of Clarke's Minimum Error Perspective Projection. The value of $M$ is however smaller than for any other Zenithal Projection considered, awth scale values true at centre. With $\beta=\frac{\pi}{2}$ we find $h=2 \cdot 04$, which does not differ much from the value $2 \cdot 105$ of the Third Projection of Parent.

From the general formulae just investigated we can find the properties of the other well-known Perspective Projections on both the tangent and Minimum Error planes. For instance

$$
\begin{aligned}
& l=0, \text { or } c=1 \text { gives the Gnomonic, } \quad r=(1-b) \tan \theta, \\
& h=\infty, \text { or } c=0 \quad, \quad \text { Orthographic, } r=(1-b) \sin \theta, \\
& h=1, \text { or } c=1 \quad, \quad \text { Stereographic, } r=2(1-b) \tan l_{2} \theta,
\end{aligned}
$$

the appropriate value of $b$ being found by substituting the proper value of $c$ in the above expression for $b$, if the projection is desired on the minimum error plane. It may be noticed that if we take $c=\frac{1}{3}$, we obtain a Perspective Projection which is approximately Equidistant, for it agrees with the latter to terms in $\theta^{2}$ and $\beta^{2}$ inclusive. And as the areal scale is to two terms expanded $a r / d \theta \cdot r / \sin \theta=\mathrm{r}+\frac{\theta^{-2}}{2}(4 c-1) \ldots$ we can get a Perspective Projection which is nearly Equal Area by taking $c=\frac{1}{4}$, or $h=3$. In this case $b=0$ to a first approximation, a result pointed out by J. I. Crais, p. 58 .

Though it has all these inherent properties the I'erspective Projection has, in the writer's opinion, been over-rated in the text-books written for practical cartographers. General Sir H. James extols it above Airy's because it is a real, or purely geometrical, projection. But as we have seen, it is in its general form awkward to compute both for plotting and for finding the scale errors, and it differs inappreciably from Minimum Error Projections of simpler form such as the Breusing (Harmonic) or the Equidistant.

\section{The Minimum Error Zenithal Projection with scale true at centre.}

The fact that Airy's solution with the constant $A=1$ does not give the Minimum Error Zenithal Projection, subject to the condition that the scale shall be true at the centre, caused the writer to try and find why the ordinary process of the Calculus of Variations failed in this instance. After solving the Perspective Projection with this stipulation it seemed clear that the requisite function when expanded would be of the form $r=\theta-{ }_{00}^{1} 6 \ldots$, because the Perspective Projection agreed so closely with Airy's in the general case. Also if we assume $r=\theta+a \theta^{*} \ldots$, where $a$ is a constant to be determined by making $M$ a minimum, we find $M={ }_{0}^{\beta a}\left(10 a^{2}+\frac{a}{3}+\frac{1}{36}\right)$, whence $a=-\frac{1}{60}$ and $I I=\frac{\beta^{6}}{240}$, arreeing so far with the Purspetive on 
tion. On substituting this solution in the fundamental formula of the Calculus of Variations

$$
\delta U=(\delta M \text { in our case })=\int_{0}^{\beta} K \delta r d \theta+H_{\beta}-H_{0},
$$

where $\quad K=N \begin{aligned} & d P \\ & d \theta\end{aligned} \ldots: I I_{\beta}=\delta r_{\beta}\left(P_{\beta}-\ldots\right) ; H_{0}=\delta r_{11}\left(P_{0} \ldots\right)$,

the equation $\delta U=0$ was found to hold, though the usual separate conditions $K^{-}=0$ and $H_{\boldsymbol{\beta}}-H_{0}=0$ did not hold.

The writer was fortunate enough to interest Professor J. W. Nicholson, l.R.S., in this question, and with his assistance the fundamental equation of the Calculus of Variations was, at any rate partially, reconciled with the above indirect solution in the following way. We have

$$
M \int_{0}^{\beta}\left((d r / d \theta-r)^{2}+\left(r_{1} \sin \theta-\mathrm{r}\right)^{2}, \sin \theta d \theta\right.
$$

and in the usual notation of that Calculus

$$
\delta M=\delta \int_{0}^{\beta} V^{\prime} d \theta=\int_{0}^{\beta}\left(N \delta \gamma+\rho^{\prime} \partial \rho\right) d \theta=0 .
$$

Now assume, can be expanded in the form $r=a \theta+b \theta^{\circ}+c \theta^{5} \ldots$, where $a, b, c \ldots$ are constants to be determined so as to fulfil the conditions prescribed. Then since $d r / d \theta=\mathrm{I}$ when $\theta=0$ we have $a=\mathrm{I}$ and

Now

$$
r=\theta+i \theta^{*}+c \theta^{3} \ldots
$$

$\lambda \bar{r} \quad \bar{c} r=2(r \sin \theta-\mathrm{I})$, and $P=\hat{c} \zeta \bar{\gamma} \quad 2 \sin \theta(d r d \theta-1)$, also

$$
p=d r d \theta \quad 1+3 b \theta^{2}+5 c \theta^{4} \ldots, \quad r / \sin \theta \quad \mathrm{I}+\left(b+\frac{1}{6}\right) \theta^{2}+\left(c+\frac{b}{6}+\frac{7}{360}\right) \theta^{4}
$$

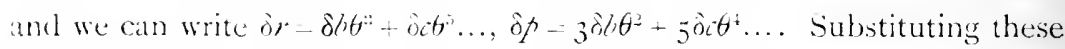

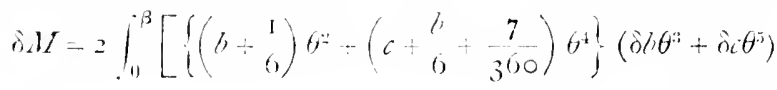

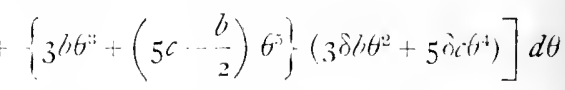

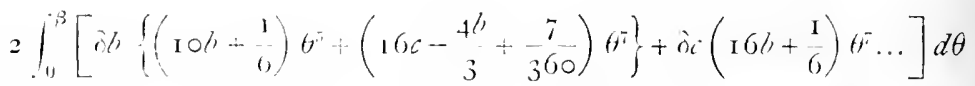

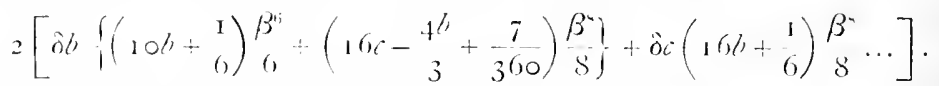

for this to ramish for erery ratrition of $b$ we must have

$$
101+\frac{1}{6}+3 \beta^{2}\left(16 c-\frac{4 k}{3}+\frac{7}{360}\right) 0
$$

and if we c an sigy that $b$ must be imlependent of $c$, then $c=0$ and

$$
1-{ }_{60}^{1}-\frac{\beta "}{320} \cdots=-{ }_{60}^{1}\left(1+\frac{3 \beta^{\prime \prime}}{16}\right)
$$


and we shall find

$$
M=\frac{\beta^{i}}{6}\left(\frac{\mathrm{I}}{40}+\frac{\beta^{2}}{1600}\right)=\frac{\beta^{6}}{240}\left(\mathrm{I}+\frac{\beta^{2}}{40} \cdots\right) .
$$

This is certainly smaller than the value of $M$ found in the Perspective Projection with scales true at centre, viz., $\frac{\beta^{4 i}}{240}\left(\mathrm{I}+\frac{\mathrm{I} 3 \beta^{2}}{200} \cdots\right)$, but the evaluation of $c$ and higher coefficients seems to leave something to be desired as a matter of principle. So far as the cartographer is concerned the formula $r=\theta-\frac{\mathrm{I}}{60} \theta^{3}$ will be accurate enough for all practical purposes, or if desired the next term in the formula of the Perspective Minimum Error Projection with scale true at centre may be used, vi $\%$,

$$
r=\theta-\frac{\theta^{3}}{60}+\frac{21 \theta^{3} \beta^{2}}{4000}-\frac{\theta^{5}}{150} \cdots
$$

This must give a result very close to the absolute minimum of average error. The theoretical difficulty is mentioned in case it may attract the notice of mathematicians interested in the Calculus of Variations, which would appear to require development in those cases where conditions are imposed on the derivatives of the function sought, at the limits.

\section{Method of evaluating the average error.}

There is another theoretical point which deserves notice. It will have been remarked that Airy and Clarke integrate the squares of the scate errors over the surface of the Earth corresponding to the map concerned. It appeared to the writer to be more natural to find the average error by integrating this quantity over the surface of the map and then to divide by the area of the map before evaluating the minimum. It appears that when once the form of the projection has been fixed it is as easy to use one method as the other. For example taking the Equidistant Projection $r=A \theta=(\mathbf{I}+b) \theta$, the scale errors will be expressed as before but the element of zone on the map will be expressed by $2 \pi r d r=2 \pi r d r d \theta$, so that we multiply the squares of scale errors by $r d r / d \theta$ instead of $\sin \theta$. In the present case $r d r d \theta=A^{*} \theta$. Our integral $M$ is however divided by

$$
2 \pi \int_{0}^{\beta} r \frac{d r}{d \theta} d \theta=2 \pi A^{*} \beta_{2} \text {. }
$$

so that the $A^{2}$ in numerator and denominator cancel out and we simply have to multiply by $\theta$ instead of $\sin \theta$ before integrating and differentiating for 6 . By using the expansion method we obtain in fact

$$
\begin{aligned}
& M=A^{2}\left[\frac{\beta^{4}}{216}\left(\mathrm{I}+\frac{7 \beta^{*}}{40}+\frac{32 \mathrm{I} \beta^{4}}{1+000}\right)+{ }_{\mathrm{I} 2}^{1+\beta^{4}}\left(1+\frac{\mathrm{I} 7 \beta^{2}}{90}+\frac{4.3 \beta^{4}}{1650}\right)\right. \\
& \left.+b^{2} \beta^{2}\left(1+\frac{\beta^{2}}{\mathrm{I}_{2}}+\begin{array}{l}
\beta^{+} \\
90
\end{array}\right)\right] \text {. } \\
& 2-2
\end{aligned}
$$


whence

$$
b=-\frac{\beta^{2}}{24}\left(1+\frac{19 \beta^{2}}{180}+\frac{43 \beta^{4}}{7560}\right)
$$

and

$$
\begin{aligned}
M & =A^{2}\left[\frac{\beta^{45}}{216}\left(\mathrm{I}+\frac{7 \beta^{2}}{40}+\frac{321 \beta^{4}}{1+000}\right)-\frac{\beta^{4}}{576}\left(1+\frac{53 \beta^{2}}{180}+\frac{11617 \beta^{4}}{226,800}\right)\right] \\
& =A^{2}\left[\frac{8 \beta^{6}}{1728}\left(1+\frac{7 \beta^{2}}{40}+\frac{321 \beta^{4}}{14000}\right)-\frac{3 \beta^{4}}{1728}\left(1+\frac{53 \beta^{2}}{180}+\frac{11617 \beta^{4}}{226,500}\right)\right] \\
& =A^{2}{ }_{1728}^{5 \beta^{4}}\left(1+\frac{31 \beta^{2}}{360} \cdots\right) ;
\end{aligned}
$$

and since the area of the map $=\frac{A^{2} \beta^{2}}{2}$ we have

as against

$$
\begin{aligned}
& \mu=\sqrt{\left(\frac{5}{864}\right) \cdot \beta^{2}\left(1+\frac{31 \beta^{2}}{600} \cdots\right)} \\
& \mu=\sqrt{\left(\frac{5}{864}\right) \cdot \beta^{2}\left(1+\frac{7 \beta^{2}}{200} \cdots\right)}
\end{aligned}
$$

found by integrating over the Earth. Integration over the map therefore gives a result for $\mu$ greater in the proportion of $1+\beta^{2} / 60: 1$ in this case. The maximum scale at the boundary is however $1+\frac{\beta^{2}}{8}+\frac{7 \beta^{4}}{86+}$ as against $1+\frac{\beta^{2}}{8}+\frac{\beta^{4}}{10 S}$ in the other method, i.e. slightly smaller. Application of this method to the other fixed functions leads to similar changes in the third term of the scale values, but when we come to apply it to the problen of finding the minimum error function the question becomes much more difficult, as the minimum value of the quotient of two integrals is required. With some valuable assistance from Prof. A. F. H. Love, F.R.S., the writer was able to pet this problem into the form of a differential equation, much more complex than Airy's, and an equation involving values at the limits. It was not found possible to solve the differential equation in terms of known functions, but it was solved in series for 3 terms and gave a result agrecing with Airy's solution to 2 terms. 'The question of finding the averase error by integrating orer the map is therefore not of practical improtance to the cartographer, but it is mentioned here as a point of theoretical interest, and also to reassure those who maty be inclined to object to solutions differing from the absolute minimum error solutions in the third term of the expansion of the functions, by the possibility that the validity of those third terms may itself be a matter of arsument.

\section{Summary of results.}

'The Minimum Error 'cenithal P'rojections have been thoroughly examined by flecturing and complaring the formulate for their mean spuare, or average, scale ermors. It is shewn that for maps of the laresest extent the Airy, larewing and charke l'erpecetive l'rojections are almost identical. It is 
shewn that the minimum error condition differs only in terms of a negligible order from the projection obtained by making the total area of the map true.

It is also shewn that the Equidistant P'rojection with total area true has an average error only about $5 \%$ greater thin the average error of Airy's, while the maximum error is the same' in both. 'This small defect is far outbalanced by the simplicity of its formula and this Projection is therefore recommended to cartographers as the best zenithal projection in all cases, except where orthomorphism or equality of area or some other special property is required.

The cases where it is desirable to use Zenithal Projections rather than Conical (of which Zenithal form a special case) will be considered later. 


\section{CHAPTER II \\ THE MINIMUM ERROR CONICAL PROJECTIONS}

We have already stated that the Zenithal Projections are only a particular case of the Conical Class, in which the map instead of being developed on a plane is developed on the surface of a cone having as axis the diameter of the sphere which passes through the centre of the zenithal map. This cone is then supposed to be laid out flat. The only difference is that the projection of a small circle on the sphere, perpendicular to the axis, instead of being a complete circle of circumference $2 \pi r$ is a portion of a complete circle $2 \pi n r$, where $n$ is the sine of half the angle at the vertex of the cone and is called the constant of the cone. 'The radial scale is as before $d r / d \theta$, but the perpendicular scale is now $n r / \sin \theta$.

The expression for $M$ becomes

$$
M=\int_{\alpha}^{\beta} V d \theta=\int_{\alpha}^{\beta}\left\{(d r d \theta-\mathrm{I})^{2}+(n r \sin \theta-1)^{2 !} ; \sin \theta d \theta,\right.
$$

and the expression for the corresponding area on the sphere is as before $(\cos \alpha-\cos \beta)$, leaving the constant multiplier $2 \pi R^{2}$ in each.

In the usual notation of the Calculus of Variations

$$
\begin{gathered}
N=\bar{c} V \partial=2 n(n \boldsymbol{r} / \sin \theta-\mathrm{I}), \quad P=\bar{c} V \bar{c} p=2 \sin \theta(d r, d \theta-\mathrm{I}), \\
d P_{\gamma} d \theta=2 \cos \theta(d r d \theta-\mathrm{I})+2 \sin \theta d^{2} \boldsymbol{r} d \theta^{\prime \prime},
\end{gathered}
$$

so that the differential equation $N-d P d \theta=0$ becomes

$$
\sin ^{2} \theta \frac{d^{2} r}{d \theta^{2}}+\sin \theta \cos \theta \frac{d r}{d \theta}-n^{2} r-\sin \theta \cos \theta-n \sin \theta \text {. }
$$

The complete solution of this equation is

$$
r=A\left(\tan \prod_{2}^{1} \theta\right)^{\prime \prime}+\left(\cot \prod_{2}^{1} \theta\right)^{\prime \prime} \int\left(\tan \prod_{2}^{1} \theta\right)^{\prime \prime} d \theta+B\left(\cot \prod_{2}^{1} \theta\right)^{\prime \prime},
$$

which at once reduces to Airy's solution for the Zenithal Class when we make $n \ldots 1$, for $\int \tan ! \theta a d \theta=2 \log \sec \frac{1}{2} \theta$. The constants $A$ and $B$ are determined from the conditions that $P=0$ both when $\theta=\alpha$ and $\theta \beta$, therefore $d r d \theta$ - I when $\theta$ a and $\theta=\beta$, or the raddial scale is true at both boundaries of the map. These two equations lead to the following values of the corstants:

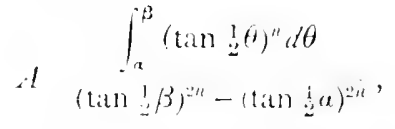

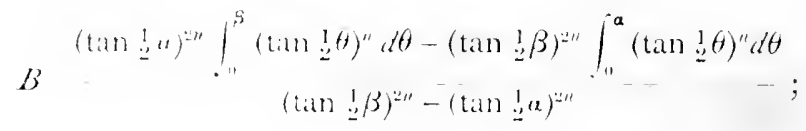


and the limiting radii of the map will be

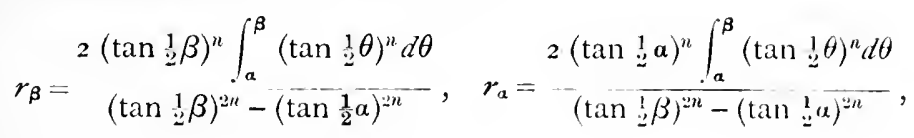

so that the depth of the map $r_{\beta}-r_{a}$

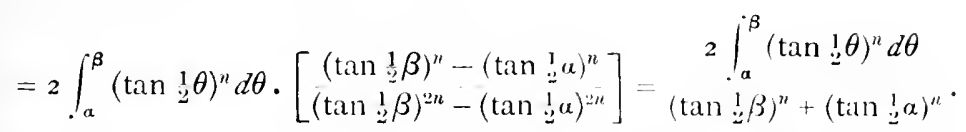

The only trouble so far is that the integral $\int\left(\tan \int_{2}^{1} \theta\right)^{\prime \prime} d \theta$ cannot be expressed in known functions except when $\|$. $\mathrm{t}$. It can be evaluated for any given value of $n$ in series or by mechanical integration. 'The calculations may appear rather formidable at first sight but they would not in any given case occupy an expert computer more than a day or two, which is a small matter compared with the total time occupied in the compilation of a map. IVe shall shew, however, that by means of expansions and simplifications even this labour can be avoided, at the expense of almost imperceptible increments of the average scale error.

We have still the constant $n$ at our disposal and in order to find the value of this which makes $X$ a minimum, it will be convenient to consider the Orthomorphic and Equal Area parts of our solution separately.

\section{Minimum Error Conical Orthomorphic Projection.}

The general formula for this is $r=2 A\left(\tan \frac{1}{2} \theta\right)^{n}$, where

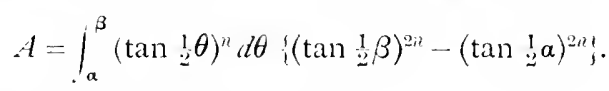

For simplicity we shall write henceforth

$$
\int_{a}^{\beta} \text { for } \int_{a}^{\beta}\left(\tan \stackrel{2}{2}^{1} \theta\right)^{n} d \theta
$$

Substituting this in the expression for $\lambda /$ formed in the usual way, we shall find after some transformations

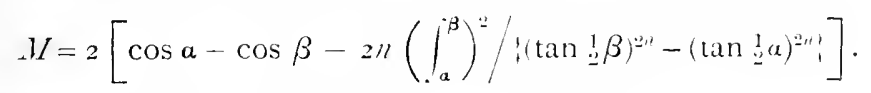

Writing $\stackrel{1}{\stackrel{2}{2}}(\beta+\alpha)=\chi$ and $\underset{2}{\stackrel{1}{2}}(\beta-\alpha)=\delta$, we have

$$
\cos \alpha-\cos \beta=2 \sin \chi \sin \delta=2 \sin \chi \cdot \delta\left(1-\frac{\delta^{2}}{6}+\frac{\delta^{4}}{\mathrm{I}_{2} 0} \cdots\right) .
$$

We can also expand the integral $\int_{a}^{\beta}$ in terms of the middle value of the function and the limits by the following general formula

$$
\int_{a}^{\beta} f(\theta) d \theta-2 \delta\left\{f(x) \div \frac{\delta^{2}}{6} f^{\prime \prime}(x)+\frac{\delta^{4}}{120^{\circ}} f^{\text {ix }}(x) \ldots\right\}
$$


In our case

$$
\begin{aligned}
& f(x)=\left(\tan { }_{2}^{1} \chi\right)^{n} ; f^{\prime \prime}(\chi)=\frac{n(n-\cos \chi)}{\sin ^{2} \chi}\left(\tan 1_{2}^{1} x\right)^{n} \\
& f^{\mathrm{ir}}(x)=\frac{n^{4}-6 n^{3} \cos x+7 n^{2} \cos ^{2} x+4 n^{2}-5 n \cos \chi-n \cos ^{3} x}{\sin ^{4} x}\left(\tan \operatorname{I}_{2} x\right)^{n} .
\end{aligned}
$$

Thus

$$
\begin{aligned}
& 2 n\left(\int_{a}^{\beta}\right)^{2}=8 n \delta^{2}\left(\tan \stackrel{?}{2}_{2} \chi\right)^{2 n}\left\{\begin{array}{c}
1+\delta^{2} n(n-\cos \chi) \\
3 \sin ^{2} \chi
\end{array}\right. \\
& \left.+\frac{\delta^{4}}{360} \cdot \frac{16 n^{4}-56 n^{3} \cos x+52 n^{2} \cos ^{2} x+24 n^{2}-30 n \cos x-6 n \cos ^{3} x}{\sin ^{4} x} \ldots\right\} \text {. }
\end{aligned}
$$

We also have

$$
\left(\tan !_{2}^{1} \beta\right)^{2 n}-\left(\tan !_{2}^{1} \alpha\right)^{2 n}=2 n \int_{\alpha}^{\beta}\left(\tan !_{2}^{1} \theta\right)^{2 n} \operatorname{cosec} \theta d \theta
$$

and expanding this in the same manner we get $\underset{\sin \chi}{2 n\left(\tan \frac{1}{2} \chi\right)^{2 n}}\left\{1+\frac{\delta^{2}}{6} \cdot{ }^{n^{2}-6 n \cos \chi+1-\cos ^{2} \chi}\right.$

$$
\begin{aligned}
& \sin \chi \quad\left\{1+6^{\circ}-\sin ^{2} \chi\right. \\
& 16 n^{4}-80 n^{\prime \prime} \cos \chi+100 n^{2} \cos ^{2} \chi+40 n^{2}-70 n \cos x-30 n \cos ^{3} \chi \\
& +\frac{\delta^{4}}{120} . \quad+\frac{18 \cos ^{2} x+\cos ^{4} x+5}{\sin ^{4} x}
\end{aligned}
$$

I)ividing out we find

$$
\begin{aligned}
& 2 n\left(\int_{\alpha}^{\beta}\right)^{2} /\left(\tan !_{2}^{1} \beta\right)^{2 n}-\left(\tan !_{2}^{1} \alpha\right)^{2 n !} \\
& =2 \delta \sin \chi\left\{\begin{array}{l}
1-\frac{\delta^{2}}{6} \cdot 2 n^{2}-4 n \cos \chi+\mathrm{I}+\cos ^{2} \chi \\
\sin ^{2} \chi
\end{array}\right. \\
& \left.+\frac{\delta^{4}}{360} \cdot \frac{4^{8} n^{4}-96 n^{3} x+52 n^{2} \cos ^{2} x-36 n^{2}+80 n \cos x-16 n \cos ^{3} x}{-3+\cos ^{2} x+7 \cos ^{4} x-5}-\cdots\right\} \text {. }
\end{aligned}
$$

Differentiating the last with respect to $n$ to find the turning value we get the equation

$$
\begin{aligned}
& -\delta^{\prime \prime}+n-4 \cos x \\
& \frac{0^{4}}{360^{\circ}} \begin{array}{c}
102 n^{3}-288 n^{2} \cos x+10+1 n \cos ^{2} x-72 n+80 \cos x-16 \cos ^{2 *} x \\
\sin ^{4} x
\end{array}=0 \text {, }
\end{aligned}
$$

whence $n$ cos $x$ to a lirst approximation, and inserting this value in the second term we ind

$$
n \cos x+\frac{\gamma^{2}}{2.40^{\circ}} \cdot \frac{8 \cos x-8 \cos ^{3} x}{\sin ^{2} x} \cos x\left(1+\delta_{30}^{\prime 2} \cdots\right) .
$$

When we incert this value of $n$ in the expression for $/ /$ we find

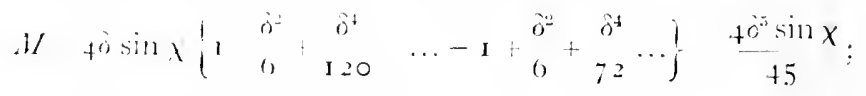

and diviling by the areal, $\mu^{2} \quad \because=0$. 
When the map extends to the centre or when $a=0$ we proceed as follows. We have

$$
M=2\left\{\mathrm{I}-\cos \beta-2 n\left(\int_{0}^{\beta}\right)^{2}\left(\cot \frac{1}{2} \beta\right)^{n n}\right\} .
$$

Now

$$
\int_{0}^{\beta}=2\left\{\frac{t^{1+n}}{1+n}-\frac{t^{3+n}}{3+n}+\frac{t^{5+n}}{5+n}-\ldots\right\},
$$

where $t=\tan \frac{1}{2} \beta$; therefore

$n\left(\int_{0}^{\beta}\right)^{2}\left(\cot \frac{1}{2} \beta\right)^{2 n}$

$$
\begin{aligned}
& =4 n\left\{\frac{t^{2}}{(1+n)^{2}}-\frac{2 t^{4}}{(1+n)(3+n)}+t^{6}\left(\begin{array}{c}
2 \\
(1+n)(5+n)^{+}+ \\
(3+n)^{2}
\end{array}\right) \ldots\right\} \\
& =4 t^{2}\left\{\frac{n}{(\mathrm{r}+n)^{2}}-\frac{2 t^{2}}{(\mathrm{I}+n)(3+n)}+t^{+}\left(\begin{array}{c}
2 \\
(\mathrm{r}+n)(5+n)^{+}
\end{array} \frac{\mathrm{I}}{(3+n)^{2}}\right) \cdots\right\} .
\end{aligned}
$$

Differentiating the part within the brackets with respect to $n$ to find the turning value we get

$$
\frac{\mathrm{I}}{(\mathrm{I}+n)^{2}}\left\{\frac{\mathrm{I}-n^{2}}{(\mathrm{I}+n)^{2}}-\frac{2 t^{2}\left(3-n^{2}\right)}{(3+n)^{2}}+t^{4}\left(\begin{array}{c}
\mathrm{I} 0-2 n^{2} \\
(5+n)^{2}
\end{array}+\begin{array}{c}
\left(9-n^{2}\right)(\mathrm{I}+n)^{2} \\
(3+n)^{4}
\end{array}\right) \ldots\right\}=0
$$

from which by successive approximation we find

$$
n=\mathrm{I}-\frac{t^{2}}{2}\left(\mathrm{I}+\frac{t^{2}}{2}\right)+\frac{25 t^{4}}{3^{6}} \cdots=\mathrm{I}-\frac{t^{2}}{2}+\frac{4 t^{4}}{9} \ldots=\mathrm{r}-\frac{\beta^{2}}{8}+\frac{\beta^{4}}{\mathrm{r}_{44}},
$$

which is very close to

as usually assumed.

$$
n=\cos ]_{2}^{1} \beta=\mathrm{I}-\frac{\beta^{2}}{8}+\frac{\beta^{4}}{3^{8}+}-\ldots
$$

Inserting the value of $n$ found in the expression for $M$ we get

$$
\begin{aligned}
M & =2\left\{\mathrm{I}-\cos \beta-2 n\left(\int_{0}^{e}\right)^{2}\left(\cot \operatorname{li}_{2} \beta\right)^{2 n}\right\} \\
& =4 t^{2}\left(\mathrm{r}-t^{2}+t^{4}-\ldots-\mathrm{I}+t^{2}-{ }_{4}^{4} t^{4} \cdots\right)=\frac{t^{6}}{\mathrm{I} 2}=\frac{\beta^{6}}{768}, \text { and } \mu=\frac{\beta^{2}}{\sqrt{3} 8_{4}},
\end{aligned}
$$

which is just half the value of $\mu$ in the zenithal system. Thus the proper choice of the constant $n$ has enabled us to find a projection which has only half the average error of a similar projection in that system. Our map is however now fan-shaped instead of circular.

\section{Minimum Error Conical (approximately) Equal Area Projection.}

Taking now the second part of our general solution we have

$$
r=2\left(\cot \frac{1}{2} \theta\right)^{n} \int\left(\tan \frac{1}{2}_{2}^{1} \theta\right)^{n} d \theta+2 B\left(\cot \log _{2}^{1} \theta\right)^{n},
$$

and on forming the expression for $M$ and differentiating for the value of $B$ to make this a minimum we shall find we must take

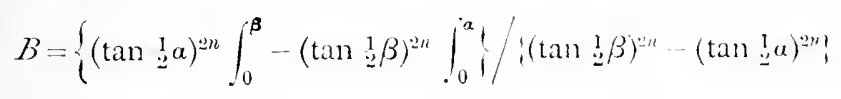


as before in the general case, and on substituting this we shall find as before that $M$ reduces to

$$
2\left[\cos \alpha-\cos \beta-2 n\left(\int_{\alpha}^{\beta}\right)^{2} /\left\{\left(\tan \frac{1}{2} \beta\right)^{2 n}-\left(\tan \frac{1}{2} \alpha\right)^{2 n}\right\}\right] .
$$

The value of $n$ will be the same as in the Orthomorphic system whether the map extends from the centre or over a zone $\alpha$ to $\beta$, i.e.

$$
n=I-\frac{\beta^{2}}{8}+\frac{\beta^{4}}{I+4} \ldots
$$

in the first case and $=\cos \chi\left(1+\frac{\delta^{2}}{3 \circ} \ldots\right)$ in the second.

And when we form the expression for $M$ in the general case

$$
r=A\left(\tan \frac{1}{2} \theta\right)^{n}+\left(\cot \frac{1}{2} \theta\right)^{n}\left(\int\left(\tan \frac{1}{2}_{2}^{1} \theta\right)^{n} d \theta+B\right)
$$

it will be found that there are no products of the several terms, and therefore the same values of $n$ will apply; also the value of $M$ will be half the value just found, viz., $M I=\frac{\beta^{6}}{153^{6}}$ for map extending to centre and $M=\frac{2 \delta^{5} \sin \chi}{45}$ for a zone.

It therefore appears that the Minimum Error Conical Projection is simply the arithmetical mean of the Minimum Error Orthomorphic and (approximately) Equal Area Projections and that all three give a map of the same total size and limiting radii, the intermediate radii in the two constituents being arranged to give orthomorphism or equality of area as the case may be and the absolute minimum error radii being the mean of the two.

\section{Numerical example of Minimum Error Map of a Zone.}

We will now illustrate our results by computing the constants and scale errors for a map extending from $a=30^{\circ}$ to $\beta=90^{\circ}$ from the pole of projection. In this case $\chi=60^{\circ}$ and $\cos \chi=\frac{1}{2}$, and we shall adopt this value for $"$ instead of the more correct value $\cos \chi\left(1+\frac{\delta^{\prime \prime}}{30} \cdots\right)$ for simplicity, and also because the term in $\delta^{2}$ does not affect the first term in the expansion for.$/$.

It will first be necessary to evaluate the function $\int(\tan \underbrace{1}_{2} \theta)^{\prime \prime} d \theta$ and this we shall do, so as to give seven equidistant values between $30^{\circ}$ and $90^{\circ}$, by Wedelles Rule. This Rule for evaluating the definite integral $\int_{n_{0}}^{\|_{6}} \|_{1} d x$ from seven efuiclistant ordinates $u_{11}, u_{1}, u_{.2}, \ldots u_{i \text { is }}$ is

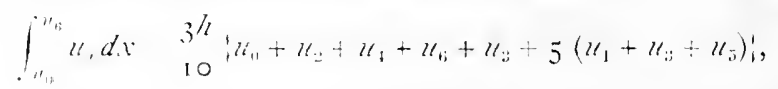


- where $h$ is the value of the interval between abscissac. It is absolutely accurate if 5 th differences are constant, and errs in excess by only ${ }_{11}^{1}{ }_{0} \Delta^{6} u_{6}$ if 6 th differences are constant. Applying this to evaluate $\int\left(\tan \frac{1}{2} \theta\right)^{\frac{1}{2}} d \theta$ for intervals of $10^{\circ}$ we tabulate $\tan \operatorname{lig}_{2}^{1} \theta$ for intervals of $0^{\circ} 50^{\prime}$ as under:

\begin{tabular}{|c|c|c|c|c|c|}
\hline & $\theta$ & $\frac{1}{2} \theta$ & $\tan \frac{1}{2} \theta$ & $u=\left(\tan \frac{1}{2} \theta\right)^{\frac{1}{2}}$ & \\
\hline o & $0^{\circ} o^{\prime}$ & $0^{\circ} 0^{\prime}$ & 0.0000 & 0.0000 & \\
\hline 1 & $1^{\circ} 40^{\prime}$ & $0^{\circ} 50^{\prime}$ & OI 45 & & $12 \mathrm{O}_{4}$ \\
\hline 2 & $3^{\circ} 20^{\prime}$ & $I^{\circ} 40^{\prime}$ & 0291 & 1706 & \\
\hline 3 & $5^{\circ} \mathrm{o}^{\prime}$ & $2^{\circ} 30^{\prime}$ & 0437 & 20190 & 2090 \\
\hline 4 & $6^{\circ} 40^{\prime}$ & $3^{\circ} 20^{\prime}$ & 0582 & $2+12$ & \\
\hline 5 & $8^{\circ} 20^{\prime}$ & $4_{0}^{\circ} 10^{\prime}$ & 0728 & & 2698 \\
\hline 6 & $100^{\circ}$ & $5^{\circ} 0^{\prime}$ & 0875 & 2958 & \\
\hline & & & $\underset{5 \times 5092}{\text { Sum }}$ & $\begin{array}{r}9166 \\
2^{\circ} 9960\end{array}$ & 5992 \\
\hline & & & & 39126 & \\
\hline
\end{tabular}

$$
\begin{gathered}
\frac{3}{10} \times 3^{\circ} 9126=1 \cdot 173^{8} . \quad \text { The interval } h=1^{\circ}+0^{\prime}=\frac{100}{3+377^{\circ}} \mathrm{c.m} . \\
\text { Integral }=\frac{1^{\circ} 1738 \times 100}{3+37^{\circ} 7}=0.0341 .
\end{gathered}
$$

Applying this method to successive intervals of $10^{\circ}$ we get the following values of the integral.

$\begin{array}{crrr}\text { Interval } & \int\left(\tan \frac{1}{2} \theta\right)^{\frac{1}{2}} d \theta & & \text { Total from o } \\ 0^{\circ}-10^{\circ} & 0.0341 & 10^{\circ} & 0.0341 \\ 10^{\circ}-20^{\circ} & 0630 & 20^{\circ} & 0971 \\ 20^{\circ}-30^{\circ} & 0821 & 30^{\circ} & .1792 \\ 30^{\circ}-40^{\circ} & 0980 & 40^{\circ} & 2772 \\ 40^{\circ}-50^{\circ} & .1123 & 50^{\circ} & .3895 \\ 50^{\circ}-60^{\circ} & .1259 & 60^{\circ} & .5154 \\ 60^{\circ}-70^{\circ} & .1393 & 70^{\circ} & .5547 \\ 70^{\circ}-80^{\circ} & .1529 & 80^{\circ} & .8076 \\ 80^{\circ}-90^{\circ} & .1671 & 90^{\circ} & .9747 \\ 90^{\circ}-100^{\circ} & .1824 & 100^{\circ} & 1.1571 \\ 100^{\circ}-110^{\circ} & .1994 & 110^{\circ} & 1.3555 \\ 110^{\circ}-120^{\circ} & 2188 & 120^{\circ} & 1.5753\end{array}$

There is one case when the definite integral $\int_{0}^{\theta}\left(\tan \int_{2}^{1} \theta\right)^{\prime \prime} d \theta$ can be expressed in known functions and that is when $\theta=\frac{\pi}{2}$, when it can be expressed by the $\psi$ functions of Gauss as $\frac{1}{2}\left\{\psi\left(\begin{array}{c}n+3 \\ +\end{array}\right)-\psi\left(\begin{array}{c}n+1 \\ +\end{array}\right)\right\}$. In this calse since

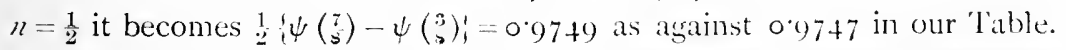
As this is only computed to 4 figures and depends on several additions the agreement is close enough. Using these results we shall find $A=1.0870$, $B=0^{\circ}$ I 29 , and we can tabulate our results as follows: 


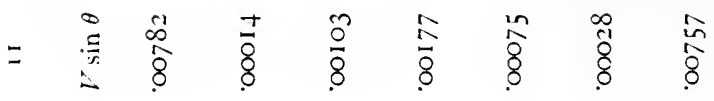

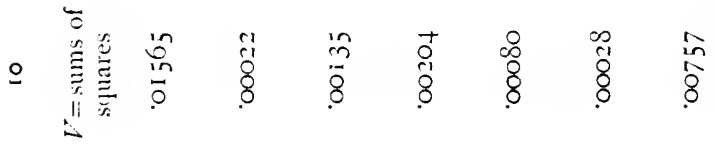

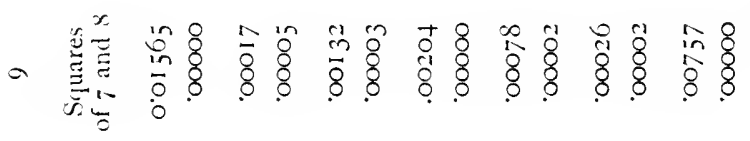

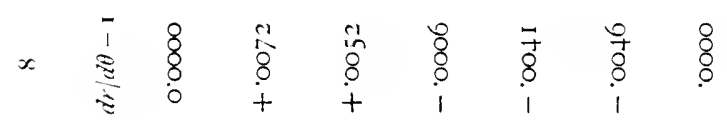

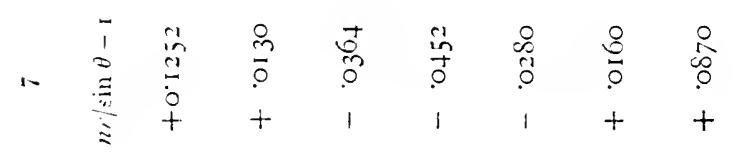

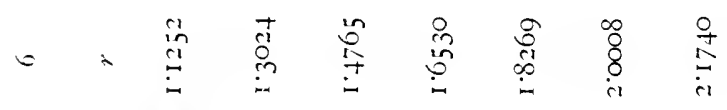

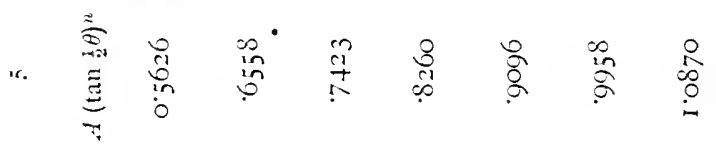

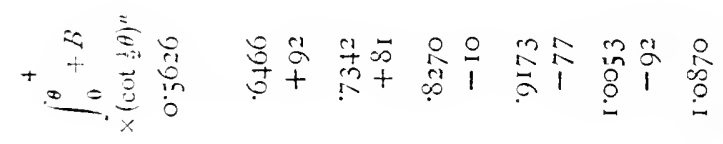

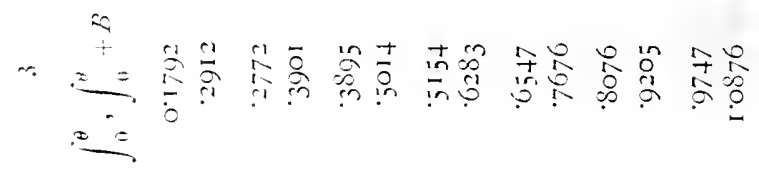

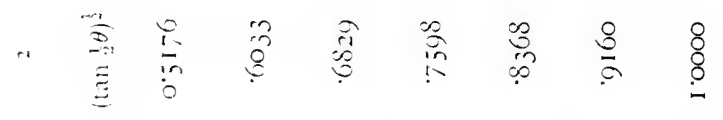

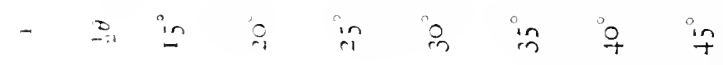


The first two columns are obvious.

The third contains the values of $\int \tan { }_{2}^{1} \theta d \theta$ just found and underneath is written the $\operatorname{sum} \int_{0}^{\theta}+B$, as this is to be multiplied by $\left(\cot \int_{2}^{1} \theta\right)^{n}$ as shewn in the fourth column.

Columns 5, 6 and 7 are obvious. Column 8 is obtained by taking the differences of columns 4 and 5 and multiplying the result by $\| r / \sin \theta$, since

$$
d r / d \theta-\mathrm{I}=\frac{n}{\sin \theta}\left\{A\left(\tan \log _{2}^{1} \theta\right)^{n}-\left(\cot \log _{2}^{n}\left(\int_{0}^{\theta}+B^{\prime}\right)\right\} .\right.
$$

These differences are set under the figures in column 4 .

Column 9 contains the squares of the scale errors, those of the radial scale being set under those of the parallel scale.

Columns 10 and 11 are obvious.

Summing column i i by Weddle's Rule we find

$$
M=\frac{0.008967 \times 10}{57.30}=0.00156 .
$$

The formula $M=\frac{2 \delta^{5} \sin }{45} \chi$ gives $0.0015 \mathrm{I}$, the difference in the last figure being probably due to taking $n=\cos \chi$ instead of $\cos \chi\left(1+\frac{\delta^{\prime \prime}}{30}\right)$. I)ividing by the area of the zone we find $\mu=0.042$. Thus we have a projection in which the maxinum scale error is +12.5 per cent. on the upper parallel, +8.7 per cent. on the lower parallel and -4.5 per cent. on the mid-parallel, while the mean square scale error is 4.2 per cent. The standard parallels will be near $40^{\circ}$ and $80^{\circ}$.

\section{Map of Hemisphere in Minimum Error Conical System.}

We will now calculate the particulars of the map of a hemisplere. Since $\alpha=0$ we put $B=0$ and taking $n=\cos \frac{1}{2} \beta=0.707$ I we find $A=0.8360$. We calculate the following Table (p. 30 ) in which the interval has leen taken as $5^{\circ}$, in order to apply Weddle's Rule.

We have thus a projection of the hemisphere in which the maximum and mean square scale errors are just about half those in Airy's Zenithal system. There is one standard parallel at about $65^{\circ}$ from the pole. 'Tlue map will be fan-shaped with a gap $=2 \pi(1-n)-104^{\circ}$ about. 'This shape of map might not be so convenient for some purposes as a circular map of a hemisphere.

\section{Simplification of formulae by making radial scale true.}

An inspection of the scale crrors in the Tables will shew that those perpendicular to greatly exceed those along the radii, particularly in the case of the zone map, where the raclial scale is true at each boundary. Iic shall therefore simplify our formulac and sacrifiee very little in aceuracy if 


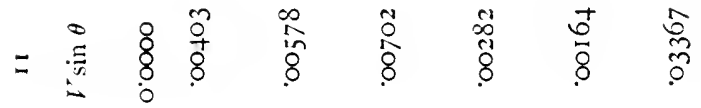

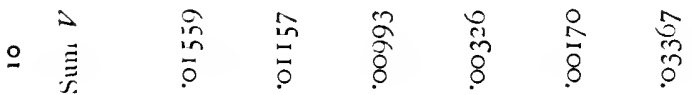

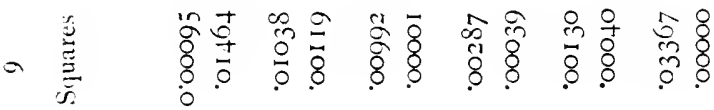

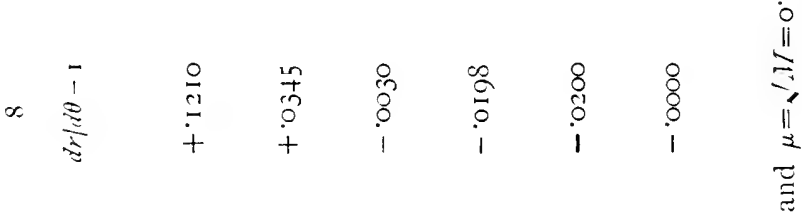

$\begin{array}{ccccccc}i & 0 & 0 & 0 & 0 & 0 & m \\ 0 & 0 & 0 & 0 & i n & 0 & 0 \\ \vdots & 0 & i & 0 & 0 & 0 & i\end{array}$

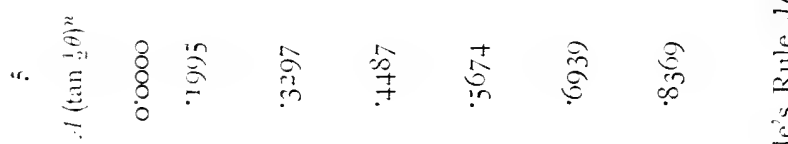

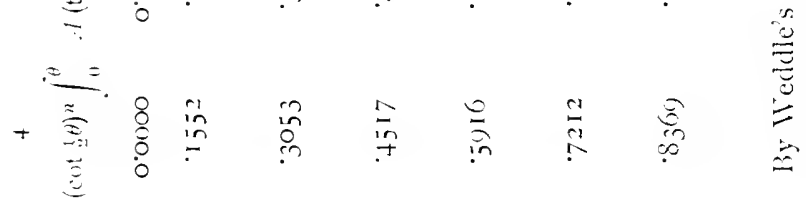

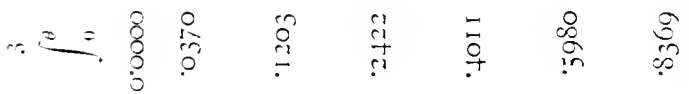

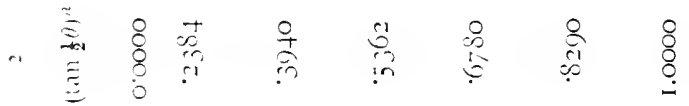

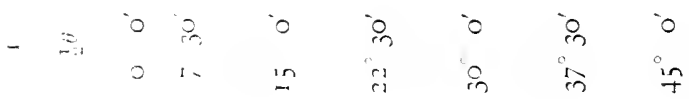


we assume that the radial scale is true always, and then determine our constants so as to make the perpendicular scale error squared a minimum.

We write therefore $r=a+\theta$, where $a$ is a constant, then

$$
d r / d \theta=\mathrm{r}, \quad n r_{1} / \sin \theta=n(a+\theta) \sin \theta,
$$

$$
\begin{aligned}
& M=\int_{\alpha}^{\beta}(n r / \sin \theta-I)^{2} \sin \theta d \theta=\left\{\cos a-\cos \beta-2 n(\beta-\alpha)\left(\alpha+\frac{1}{2}(\beta+\alpha)\right)\right.
\end{aligned}
$$

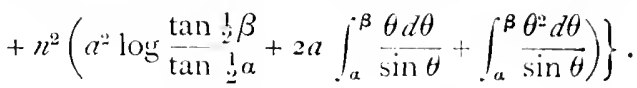

Unfortunately the integrals $\int \theta \operatorname{cosec} \theta d \theta$ and $\int \theta^{2} \operatorname{cosec} \theta d \theta$ cannot be expressed in known functions. They could if necessary be evaluated by their expansions or by mechanical integration, but calling them for a moment $S_{1}$ and $S_{2}$ and putting $d M d n=0$, we find the turning value

$$
n=(\beta-a) \cdot \frac{a+\frac{1}{2}(\beta-a)}{a^{2} \log _{\tan \frac{\tan }{2} \beta} \frac{1}{2}+2 a S_{1}+S_{:}} .
$$

Putting next $d M / d a=0$, we find

$$
a=\frac{\beta-u-n S_{1}}{n \log \left(\begin{array}{l}
\tan 1 \\
\hdashline \\
\tan \beta \\
2
\end{array}\right)} .
$$

Substituting this in the expression for $n$, we find after some reduction

$$
n=(\beta-\alpha) \cdot \frac{\frac{1}{2}(\beta+\alpha)\left(\log \tan \frac{1}{2} \beta-\log \tan \operatorname{lag}_{2}^{1} \alpha\right)-S_{1}}{S_{2}\left(\log \tan \frac{1}{2} \beta-\log \tan \frac{1}{2} \alpha\right)-S_{1}^{2}},
$$

whence $\quad M=\cos a-\cos \beta-n(\beta-\alpha)\left(a+\frac{1}{2}(\beta+\alpha)\right)$.

If the map extends to the centre then $a=0$ and $\alpha=0$. The quantity $\log \tan \frac{1}{2} \beta / \log \tan \frac{1}{2} \alpha$ becomes infinite, so dividing the numerator and denominator of the right hand side of above expression for $n$ by it, we find

$$
n=\frac{1}{2} \beta^{2} S_{2} ; \quad M=\mathrm{I}-\cos \beta-\frac{1}{4} \beta^{4} / S_{2} .
$$

Now

$$
S_{2}=\int_{0}^{\beta} \theta^{2} \operatorname{cosec} \theta d \theta-\frac{\beta^{2}}{2}+\frac{\beta^{4}}{2+}+\frac{7 \beta^{3}}{2160}+\ldots \text {, }
$$

therefore

$$
\begin{aligned}
& n=\mathrm{I}-\frac{\beta^{2}}{\mathrm{I} 2}+\frac{\beta^{4}}{2 \mathrm{I} 60}-\frac{\beta^{15}}{90720}+\ldots, \\
& M=\frac{\beta^{2}}{2}-\frac{\beta^{4}}{24}+\frac{\beta^{6}}{720}-\frac{\beta^{4}}{40320}+\ldots-\frac{\beta^{2}}{2}+\frac{\beta^{4}}{2+}-\frac{\beta^{6}}{4320}+\frac{\beta^{4}}{181,470}-\cdots \\
& 86_{4}^{-1}\left(\mathrm{I}-\beta^{2} \cdots\right)
\end{aligned}
$$

which is $2 / 5^{\text {ths }}$ the value of $M$ in the Equidistant Zenithal system.

If the map extends over a zone from $\theta-\alpha$ to $\theta=\beta$, the expressions for $n, a$ and $M$ can be simplified by expanding as follows, for which method the writer is indebted to Dr T. J. I'A. Bromwich, F.R.S. P'utting

$$
\frac{1}{2}(\beta+a)=\chi \text { and } \frac{1}{2}(\beta-u)=\delta \text {, }
$$


let

$$
\mathrm{I} / \sin (\chi-\epsilon)=A_{0}+A_{1} \epsilon+A_{2} \frac{\epsilon^{2}}{2 !}+A_{3} \frac{\epsilon^{3}}{3 !}+\ldots
$$

so that

$$
\begin{aligned}
& A_{0}=\frac{\mathrm{I}}{\sin \chi} ; \quad A_{1}=\frac{\cos \chi}{\sin ^{2} \chi} ; \quad A_{2}=\frac{\mathrm{I}+\cos ^{2} \chi}{\sin ^{3} \chi} ; \quad A_{3}=\frac{\cos \chi\left(5+\cos ^{2} \chi\right)}{\sin ^{4} \chi} ; \\
& A_{4}=\frac{\mathrm{I}}{\sin ^{5} \chi}\left(5+18 \cos ^{2} \chi+\cos ^{4} \chi\right),
\end{aligned}
$$

and so on: further write

$$
\begin{aligned}
& L=\int_{-\delta}^{+\delta} \sin (\chi-\epsilon)=2 \delta\left(A_{0}+A_{22} \frac{\delta^{2}}{6}+A_{4} \frac{\delta^{4}}{\mathrm{I} 20}+\ldots\right), \\
& M=\int_{-\delta}^{+\delta} \frac{\epsilon d \epsilon}{\sin (\chi-\epsilon)}={ }_{3}^{2 \delta^{3}}\left(A_{1}+A_{3} \frac{\delta^{2}}{10}+A_{5} \frac{\delta^{4}}{280}+\ldots\right),
\end{aligned}
$$

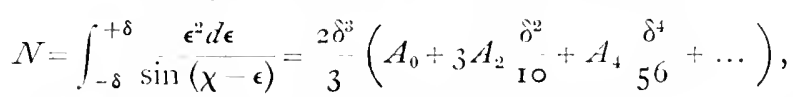

then $n=\frac{2 \delta M T}{L N^{2}-M^{2}}$. Substituting and reducing we find

$$
\begin{aligned}
& n=\cos \chi\left(\mathrm{I}+\frac{\delta^{2}}{30} \ldots\right), \quad a=\tan \chi\left(1-\frac{\delta^{2}}{5}\right)-\chi, \\
& M=2 \sin \chi\left(\delta \cdots \frac{\delta^{3}}{6}+\frac{\delta^{5}}{120} \cdots-\delta+\frac{\delta^{3}}{6}+\frac{\delta^{5}}{72}\right)=2 \sin \chi \frac{\delta^{5}}{45},
\end{aligned}
$$

which values for $n$ and $M$ agree with those found for the $M$ inimum Error solution so far as our expansions go. We are therefore justified in rejecting

\begin{tabular}{|c|c|c|c|c|c|c|c|}
\hline I & 2 & 3 & 4 & 5 & 6 & 7 & $\delta$ \\
\hline$"$ & $\begin{array}{c}\theta \text { in c.m. } \\
\theta \div \alpha\end{array}$ & $\sin \theta$ & $m \% \sin \theta$ & $n \% / \sin \theta-1$ & $\begin{array}{l}\text { Squares } \\
V\end{array}$ & $l^{+} \sin \theta$ & $\begin{array}{l}n \% \sin \theta-1 \\
\text { in . Minimum } \\
\text { Error } \\
\text { System }\end{array}$ \\
\hline 30 & $\begin{array}{l}0.5236 \\
1.1135\end{array}$ & 0.5000 & $1 \cdot 1237$ & +1237 & 0.015301 & 0.007650 & +0.1252 \\
\hline fo & $\begin{array}{r}6,81 \\
1.2480\end{array}$ & $6+28$ & 1.0110 & +.0110 & 121 & 78 & +.0130 \\
\hline 50 & $\begin{array}{l}8-27 \\
1+4,26\end{array}$ & 7660 & . 1603t & $-\cdot 0366$ & 1340 & 1026 & $-0^{0} 6_{4}$ \\
\hline 6 & $\begin{array}{l}10472 \\
10,371\end{array}$ & .8660 & అ5 540 & $-{ }^{\circ} \mathrm{O} 600$ & 2116 & 1832 & -0452 \\
\hline 20 & $\begin{array}{l}1.2217 \\
18116\end{array}$ & ()3377 & 9728 & -0272 & 740 & (x) 5 & -0280 \\
\hline (is) & $\begin{array}{l}1 \cdot 3 y y_{3} \\
19,66)_{2}\end{array}$ & $4 x^{2}+8$ & $10: 78$ & +.0178 & 317 & 312 & +0160 \\
\hline$(n)$ & $15=0$ & 1.0000 & $1 \cdot 012$ & $+{ }^{\circ} \mathrm{OrjO} 2$ & $8+3^{6}$ & 8135 & $+0 S_{70}$ \\
\hline
\end{tabular}
the rather complex formulae of that solution and adopting the uniform radial scale.

We will now illustrate our results by calculating the particulars of the same conal map as before, extending from $30^{\circ}$ to $90^{\circ}$ from the pole of projection. Putting $\delta=30^{\prime}=0.5236 \mathrm{~cm}$. and $x=60^{\circ}$, we find $a=0.5899$, $n-0.5046$, and our 'lable stands as under: 
The columns are selfexplanatory and in the last are given the perpendicular scale errors in the absolute Minimum Error Projection, for comparison with those in column 5. By Weddle's Rule we find $M=0.00159$ as against $0.0015^{6}$ in that system. The formula to first term gives $M=0.0015 \mathrm{I}$. We see that the map has two standard parallels and that the scale error at the mid-parallel $60^{\circ}$ is

$$
n(a+\chi) / \sin \chi-\mathrm{I}=\left(\mathrm{I}+\frac{\delta^{\prime \prime}}{30}\right)\left(\mathrm{I}-\frac{\delta^{2}}{5}\right)=-\frac{\delta^{2}}{6} .
$$

To find approximately where the standard parallels lie, let $\gamma$ be their angle from the centre, then $n(a+\chi-\gamma)=\sin (\chi-\gamma)$, therefore

$$
\cos \chi\left(1+\frac{\delta^{2}}{30}\right)\left(\tan \chi\left(1-\frac{\delta^{2}}{5}\right)-\gamma\right)=\sin \chi\left(1-\frac{1}{2} \gamma^{2}\right)-\gamma \cos \chi,
$$

whence

$$
\gamma= \pm \frac{1}{\sqrt{3}} \delta= \pm 0.58 \delta
$$

that is, the standard parallels lie at approximately one-fifth the depth of the map from the upper and lower edges.

It will be observed that the mean square scale error is independent of the distance from the pole of projection, or what we may call the mean co-latitude of the map, using co-latitude in a general sense as angle from the pole. This is an important result, as it affords us great liberty of choice in fitting any particular country to a map in this system. We have only to choose our pole so that the depth of the map zo shall be as small as possible, and as the choice of mean co-latitude $\chi$ crables us to select almost any curvature for our parallels, we can suit almost any shape of country. Hammer has published Tables to facilitate the construction of Conical Projections with pole in any position, but when the pole has a skew-position it will be found that generally the meridians and parallels are lines of contrary flexure and the map has an ugly appearance. Practically: speaking the only useful positions are when the pole corresponds with the terrestrial poles or when it is at an infinite distance in the perpendicular direction, leading to transverse cylindrical projections.

\section{Minimum Error Plate-Carrée Projection.}

When the mid-parallel in the Equidistant Conical Projection is of colatitude $90^{\circ}$ the projection becomes cylindrical, and if the pole of projection is at infinity in the direction of the terrestrial poles it is called the PlateCarrée Projection. When, howerer, the pole is at infinity in the transerese direction the projection becomes the Cassini l'rojection. In both calses can these projections be improved by use of a suitable seale constant.

Considering the l'late-Carrée Projection, let us now count the angle: $\theta$ from the equator instead of from the pole. The pumtity $n$ incomes sero. The parallel scale error, if the cylinder is tangent at the equater, is repre- 
sented by $\sec \theta-$ r. Let now $A$ be a scale constant and let us determine $A$ so that

$$
2 \int_{0}^{\delta}(A \sec \theta-\mathrm{I})^{2} \cos \theta d \theta=2\left[\frac{A^{2}}{2} \log \frac{\mathrm{I}+\sin \delta}{1-\sin \delta}-2 A \delta+\sin \delta\right]
$$

shall be a minimum. We shall find

$$
\begin{gathered}
A=2 \delta,\{\log (1+\sin \delta)-\log (1-\sin \delta)\}=1-\frac{\delta^{2}}{6}-\frac{\delta^{4}}{72} \cdots ; \\
M=2\left[\sin \delta-2 \delta^{2} /\{\log (1+\sin \delta)-\log (1-\sin \delta)\}\right]=\frac{2 \delta^{5}}{45} \text { and } \mu=\frac{\delta^{2}}{\sqrt{45}} .
\end{gathered}
$$

These are precisely the results which we should have obtained by making $x 90^{\circ}$ in our general formula.

If we take $A=$, or make the cylinder tangent at the equator,

$$
M=2\left\{\sin \delta-2 \delta+\frac{1}{2} \log \frac{1+\sin \delta}{1-\sin \delta}\right\}=\frac{\delta^{\prime}}{10} ; \mu=\frac{\delta^{2}}{\sqrt{ } 20} .
$$

By creating two standard parallels instead of one, we have introduced a negative seale error along the middle parallel of $-{ }_{6}^{\delta^{*}}$, we have reduced the mean square scale error in the ratio $\sqrt{ }+5: \sqrt{ } / 20=6 \cdot 7: 4 \cdot 5$, and the maximum scale at the extreme parallels has been reduced from sec $\delta=1+\frac{\delta^{2}}{2} \ldots$ to

$$
\left(1+\frac{\delta^{\prime 2}}{2}\right)\left(1-\frac{\delta^{2}}{6}\right)=1+\frac{\delta^{2}}{3} \cdots
$$

\section{Minimum Error Cassini Projection.}

Thissut has pointed ont (1). I 62) that the Cassini Projection is simply the I'late-Carrece turned through a right angle, so that what was the equator beconses the principal meridian. Our result above shews that by reducing the scale along this meridian by one-third the scale cror at the extreme cast or west edge of the map, creating what we may call two standard psendo-meridians (or sul)-parallels), we reduce the mean syuare error of the map to a minimum. A device of this sort is used in the projection of the International Map) on the scale of $\mathrm{I}$ /million. The scale error on the principal meridian is made ecual and opposite to that on the extremes, i.c. the scale constimt used is $1-\frac{\delta^{\prime \prime}}{4}$ instcad of $1 \frac{\delta^{2}}{6}$.

\section{Minimum Error Mercator Projection.}

The work we have junt done enables us to find the Minimum Error Iterator Projection which is simply the Cylindrical Orthomorplic. In this 
the radial or meridian scale instead of 'being uniform is the same as the parallel scale. Using $\left(r-r_{\pi}\right)$ as distance from equator, we have

$$
d\left(r-r_{\pi}\right) / d \theta=\sec \theta,
$$

which gives $\quad\left(r-r_{\frac{\pi}{2}}\right)=\log \tan \left(\frac{\pi}{4}+\frac{1}{2} \theta\right)=\frac{1}{2} \log \frac{1+\sin \theta}{1-\sin \theta}$.

The expression for $M$ is simply double that in the Plate-Carréc, the constant $A$ is the same, but must be applied to the perpendicular as well as the meridian coordinates, and $\mu$ is $\sqrt{ } 2$ times as great $\sqrt{45} \cdot{ }^{2} \cdot \delta^{2}=\delta^{2} \cdot 74$.

\section{Minimum Error Conformal Projection of Gauss.}

As the Cassini is simply the Plate-Carrée turned through a right angle, so the Conformal Projection of Gauss, used for the survey of Hanover and more recently for the survey of Egypt, is simply the Mlercator applied to a meridian. We see therefore that its mean square scale error can be reduced, by multiplying both coordinates by a factor $A=1-\frac{\delta^{2}}{6}-\frac{\delta^{+}}{72} \ldots$, in the ratio $6.7 \mathrm{I}: 474$ and its maximum scale error in the ratio $3: 2$. The liniting depth of the rectified Mercator or width of the rectified Gauss Conformal is $A \frac{1}{2} \log \frac{1+\sin \delta}{1-\sin \delta}=\delta$, the same as in the Plate-Carrée or Cassini-Soldner.

IVe shall now compare our Minimum Error Equidistant Conical Projection with some others of the same class. The first of these is:

\section{Euler's Map for Russia with two standard parallels.}

This projection is described in detail in Germain, pp. $179-183$. It has the property that the absolute errors on the extreme parallels are equal, and also equal to the error of opposite sign on the mid-parallel. This leads in our notation to the two equations

$$
n(a+\chi-\delta)-\sin (\chi-\delta)=\sin \chi-n(a+\chi)=n(a+\chi+\delta)-\sin (\chi+\delta),
$$

whence

$$
\begin{gathered}
n=\cos \chi \sin \delta / \delta=\cos \chi\left(1-\frac{\delta^{2}}{6}+\frac{\delta^{4}}{120} \ldots\right), \\
a+\chi=\tan \chi \frac{1}{2} \delta \cot \frac{\delta}{2} \delta=\tan \chi\left(1-\frac{\delta^{*}}{\mathrm{I}_{2}}-\frac{\delta^{+}}{720} \ldots\right) .
\end{gathered}
$$

The map extends from co-latitude $20^{\circ}$ to co-latitude $50^{\circ}$ and Eulur finds by a calculation not so direct as ours $11-0.8098, a-0.085 j+5.3^{\prime} 2 z$ '. As this value of $a$ is so nearly $5=0.0873$ livuler adepts the latter value and so fails to reproduce one of his conditions, viz., that of eppullity of errors at middle and extremes. The following Tables give the particulars of this map calculated from the Minimum Error formula, from Euler's own constants, and from the correct values of Euler's constints. 
Minimum Error Formulae.

$$
n=\cos \chi\left(\mathrm{I}+\frac{\delta^{2}}{3^{\circ}}\right)=0.82 \mathrm{I} 56, \quad a=\tan \chi\left(\mathrm{I}-\frac{\delta^{2}}{5}\right)-\chi=0.07927 .
$$

$\begin{array}{lllll}\theta & \theta \text { in c.m. and } r & \sin \theta \quad n r / \sin \theta & n r / \sin \theta-1 & V=\text { Squares } \quad r \sin \theta\end{array}$

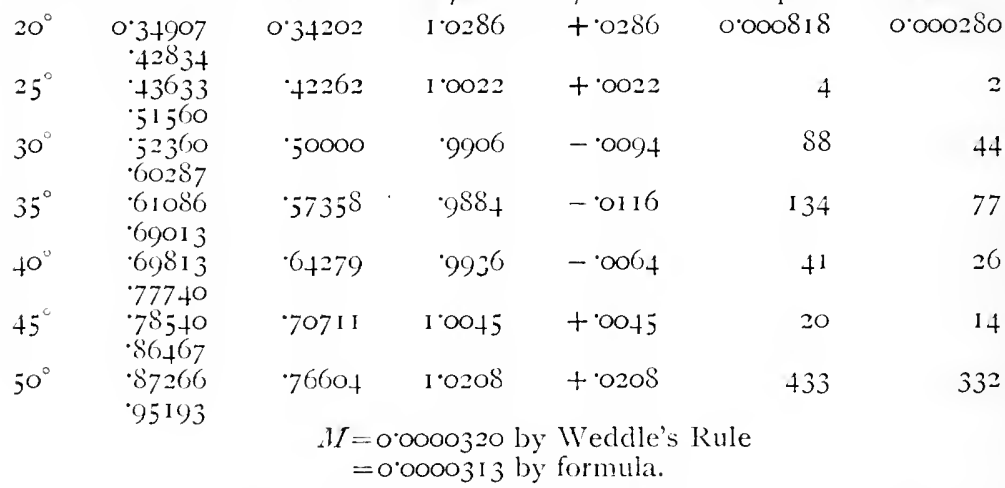

Same Map with Euler's constants, $n=0.8098, a=0.08727$.

\begin{tabular}{|c|c|c|c|c|c|}
\hline $\begin{array}{rr}20^{\circ} & 0^{\circ} 3+907 \\
& 43634\end{array}$ & 0.34202 & 1.0321 & +0321 & 0.001030 & $352+(0.0110)$ \\
\hline $\begin{array}{r}+3633 \\
.52360\end{array}$ & +2262 & 1.0032 & +.0032 & 10 & 4 \\
\hline $\begin{array}{l}5.5360 \\
.61087\end{array}$ & 50000 & 9895 & -.0105 & I IO & 55 \\
\hline $\begin{array}{l}61086 \\
69813\end{array}$ & 57358 & 9857 & $-\circ \mathrm{OI}+3$ & 204 & I $17-\left(0^{\circ} 0082\right)$ \\
\hline $\begin{array}{l}69813 \\
785+0\end{array}$ & 64279 & 9894 & - o & II 2 & 72 \\
\hline $\begin{array}{r}785+0 \\
.87267\end{array}$ & 70711 & 9994 & -.0006 & o & o \\
\hline $\begin{array}{l}87267 \\
95993\end{array}$ & $\begin{array}{c}76604 \\
M=\end{array}$ & I.OI 47 & $+\cdot 01+7$ & 216 & $165+(0.0112)$ \\
\hline
\end{tabular}

If Euler's assumption had been reproduced we should have the scale errors at extreme and midparallels, multiplied by respective sines of $\theta$, equal, whereas we have the figures shewn in brackets in the last column.

Same Map with Euler's correct constants, $n=0.8098, a=0.08535$.

\begin{tabular}{|c|c|c|c|c|c|c|}
\hline$\theta$ & "in c.m.and, & $\sin \theta$ & $m r \sin \theta$ & $m \mu \sin \theta-1$ & $I^{\prime}=$ Stquares & $l \sin \theta$ \\
\hline 20 & $\begin{array}{r}0.3+907 \\
+3+42\end{array}$ & 0.34202 & $1 \cdot 0285$ & +0.0285 & 0.000812 & $0.000278+(0.00976)$ \\
\hline 25 & $\begin{array}{l}43^{6} 34 \\
321(x)\end{array}$ & 42262 & 000 & -.0004 & o & o \\
\hline 30 & $\begin{array}{l}52360 \\
70605\end{array}$ & 50000 & .9863 & -0137 & 185 & リว \\
\hline 35 & $\begin{array}{l}61006) \\
6(x) 2528\end{array}$ & 5735 & 0.30 & -.0170 & $2 s i)$ & $166-0.009757$ \\
\hline 10 & $\begin{array}{l}6813 \\
-783+6\end{array}$ & $6+2-10$ & $0 \times 1$ & - OI29 & 1660 & 107 \\
\hline+7 & $\begin{array}{l}785+3 \\
8-075\end{array}$ & 70711 & 9072 & -0028 & 8 & 6 \\
\hline & $\begin{array}{l}x-2 f_{n} \\
x=1,601\end{array}$ & $\because 6 r i o+$ & 10128 & +0128 & $I^{2}+4$ & $125+000,80$ \\
\hline
\end{tabular}


The assumptions made are now very nearly reproduced, but, as we see, Euler by using erroneous values of his constants, was fortunate enough to reduce his mean scale error.

To calculate the formula for $M$ or $\mu$ we should strictiy speaking substitute the values of $n$ and $a$ in the equation

$$
\begin{aligned}
& M=\cos \alpha-\cos \beta+n^{2}\left(a^{r} \log \frac{\tan \tan _{2}^{1} \beta}{\operatorname{la}_{2} \alpha}+2 \alpha \int_{\alpha}^{\beta} \theta \operatorname{cosec} \theta d \theta+\int_{\alpha}^{\beta} \theta^{2} \operatorname{cosec} \theta d \theta\right) \\
& -2 n(\beta-a)\left(a+\frac{1}{2}(\beta+a)\right),
\end{aligned}
$$

but as we know $\mu$ is to the first term independent of the co-latitude we are entitled to take the value for $\chi=\frac{\pi}{2}$ and write

$$
\begin{aligned}
& M=2\left\{\sin \delta-2 A \delta+\frac{1}{2} A^{2} \log \frac{\mathrm{I}+\sin \delta}{\mathrm{i}-\sin \delta}\right\} \sin \chi \\
& =2 \delta\left\{\mathrm{I}-\frac{\delta^{2}}{6}+\frac{\delta^{4}}{\mathrm{I} 20}-\ldots-2 A+\frac{1}{2} A^{2}\left(\mathrm{I}+\frac{\delta^{2}}{6}+\frac{\delta^{4}}{24}\right)\right\} \sin \chi .
\end{aligned}
$$

Now $A$ we know is the scale at mid-parallel

$$
=n(a+\chi) / \sin \chi=\frac{1}{2} \sin \delta \cot \frac{1}{2} \delta=\mathbf{I}-\frac{\delta^{2}}{4}+\frac{\delta^{4}}{4^{8}} .
$$

Substituting this we find

$$
M=\frac{7 \delta^{5}}{120} \sin \chi ; \mu=\sqrt{\left(\frac{7}{240}\right) \delta^{2} .}
$$

Hinks, pp. 83-87 (following Close, Textbook of Topographical Surveying, p. ro7 et seq.), considers five different assumptions for this projection including Euler's (Case I). We will now exemplify his Case III in which the Scale Errors (not the absolute errors) along extreme parallels are made equal and opposite to that along the mid-parallel. The maximum negative scale error does not occur exactly on the mid-parallel, except when $\chi=90^{\circ}$, but it is very near to it, so that Hinks' Case $V$ is practically the same as his Case III. We have then the following two equations

$$
\frac{n(a+\chi-\delta)}{\sin (\chi-\delta)}-\mathrm{I}=\frac{\mathrm{I}-n(a+\chi)}{\sin \chi}-\frac{n(a+\chi+\delta)}{\sin (\chi+\delta)}-\mathbf{1},
$$

whence we get

$$
\begin{aligned}
n=\cos \chi \cdot 2 \tan \frac{1}{2} \delta \delta & =\cos \chi\left(\mathrm{I}+\frac{\delta^{2}}{\mathrm{I} 2}+\frac{\delta^{4}}{\mathrm{I} 20} \cdots\right), \\
a+\chi=\tan \chi \cdot \delta \cot \delta & =\tan \chi\left(\mathrm{I}-\frac{\delta^{2}}{j}+\delta^{4} \ldots\right) .
\end{aligned}
$$

Scale at mid-parallel $=2 \tan \sum_{2}^{1} \delta \cot \delta=1-\frac{\hat{o}^{-4}}{+}-\frac{\delta^{4}}{2+}-\ldots$ the same as in Euler's map; the value of $I /$ in this projection will therefore be the same as in Euler's to first term $=\frac{7 \delta^{5} \sin x}{120}$. We find $n-0.8238: a=0.0733$, and Table as follows : 


\begin{tabular}{|c|c|c|c|c|c|c|}
\hline $\begin{array}{c}\theta \\
20^{\circ}\end{array}$ & $\begin{array}{c}\theta \text { in c.m. and } r \\
0.3+91 \\
.4224\end{array}$ & $\begin{array}{c}\sin \theta \\
0.3420\end{array}$ & $\begin{array}{l}n r / \sin \theta \\
\text { rol } 74\end{array}$ & $\begin{array}{l}n r / \sin \theta-\mathrm{r} \\
+0.0174\end{array}$ & $\begin{array}{c}V=\text { Squares } \\
0^{\circ} 000303\end{array}$ & $\begin{array}{c}V \sin \theta \\
0.000104\end{array}$ \\
\hline $25^{\circ}$ & $\begin{array}{r}4363 \\
+5096\end{array}$ & 4226 & 9934 & - 0066 & +3 & IS \\
\hline $30^{\circ}$ & $\begin{array}{l}5236 \\
5969\end{array}$ & 5000 & 9834 & - .0166 & 275 & I 37 \\
\hline $35^{\circ}$ & $\begin{array}{l}6109 \\
.6842\end{array}$ & $\cdot 5736$ & 9826 & -0174 & 303 & 174 \\
\hline $40^{\circ}$ & $\begin{array}{l}6981 \\
7714\end{array}$ & $6+28$ & 9886 & - 0114 & 130 & $8_{4}$ \\
\hline $45^{\circ}$ & $\begin{array}{l}7854 \\
\cdot 8587\end{array}$ & 7071 & 1.0003 & $+\mathrm{OOOO}_{3}$ & o & o \\
\hline $50^{\circ}$ & $\begin{array}{l}8727 \\
.9460\end{array}$ & 7660 & 1.0173 & +0173 & 299 & 229 \\
\hline
\end{tabular}

- The position of the standard parallels in this, and in all cases where the scale error on mid-parallel is equal and opposite to those on extreme parallels, is given by $\theta_{s}^{2}=\int_{2}^{1} \delta^{2}$, i.e. about one-seventh the depth of the mal' from edges.

We will now discuss two remarkable projections of this class invented by the Rev. Patrick Murdoch, F.R.S., and described by him in a paper published in the Plitosophical Transactions of the Royal Society for $175^{8 .}$ But first we will investigate the condition that the total area of the map shall be equal to the area on the sphere which it represents. The area of a zone on the sphere between co-latitudes $a$ and $\beta$ is

$$
2 \pi R^{2}(\cos \alpha-\cos \beta)=2 \pi R^{\prime \prime 2} 2 \sin \delta \sin \chi .
$$

The corresponding area on the map is

$$
n \pi\left(r_{\beta}^{2}-r_{a}^{2}\right)=n \pi\left(r_{\beta}+r_{\alpha}\right)\left(r_{\beta}-r_{\alpha}\right)=2 n \pi R^{22} .2 \delta(a+\chi) .
$$

Therefore for equality of areas $n \delta(a+\chi)=\sin \delta \sin \chi$, or

$$
n(a+\chi) / \sin \chi=\sin \delta / \delta=\mathrm{I}-\frac{\delta^{2}}{6}+\frac{\delta^{t}}{\mathrm{I} 20} \ldots
$$

Thus the Minimum Error Conical P'rojection has to a first approximation its total area true, as in the zenithal system.

\section{Murdoch's First Projection.}

Murdoch's Paper is very concise and he does not give the full proof of his result. lle takes $n=\cos \chi$ simply, and then gives a rule for drawing the map which in effect amounts to taking $a+\chi-\tan \chi \sin \delta / \delta$, and this we see is the necusiary condition.

Murdoch is result wats afterwards taken up by the (ierman mathematicians, Tobias Mayer and Albers (sce (iermain, 1). 185 ). The former professed to have diserevered some flaw in Mureloch's argment, and the latter clatiorated the result and atso reveloped his own Equal Area Projection of this class in which the ratial scale is of rourse no longer uniform. The writer has 
examined Murdoch's original Paper and can discover no faw in his statements; and it is remarkable that Murdoch by a kind of intuition hit upon a projection which has to a first approximation the same mean square scale error as the absolute Minimum Error l'rojection of its class.

For if we substitute $A=\mathrm{I}-\frac{\delta^{2}}{6}+\frac{\delta^{4}}{120}$ in the formula

$$
M=2 \delta\left\{1-\frac{\delta^{2}}{6}+\frac{\delta^{4}}{120}-2 A+\frac{1}{2} A^{2}\left(1+\frac{\delta^{2}}{6}+\frac{\delta^{4}}{24}\right)\right\} \sin \chi,
$$

we find $M=\frac{2 \delta^{\bar{j}}}{45} \sin \chi$ as before.

To exemplify this we will calculate the map from co-latitude $30^{\circ}$ to co-latitude $90^{\circ}$ on Murdoch's First P'rojection. Wie shall finel

$$
n=\cos 60^{\circ}=0.5000, \quad a=\tan \chi \sin \delta \delta-\chi=0.6068 .
$$

\begin{tabular}{|c|c|c|c|c|c|c|}
\hline $\begin{array}{c}\theta \\
30^{\circ}\end{array}$ & $\begin{array}{c}\theta \text { in } \mathrm{c} . \mathrm{m} \text {. and } r \\
0.5236 \\
\mathrm{I}^{\cdot} \mathrm{1} 3 \mathrm{3}+4\end{array}$ & $\begin{array}{c}\sin \theta \\
0.5000\end{array}$ & $\begin{array}{l}n \% / \sin \theta \\
\mathrm{I}_{3} \mathrm{O}_{+}\end{array}$ & $\begin{array}{c}m \% / \sin \theta-1 \\
+0^{\prime} 130_{4}\end{array}$ & $\begin{array}{c}V=\text { Squares } \\
0.017004\end{array}$ & $\begin{array}{c}V \sin \theta \\
0.008502\end{array}$ \\
\hline $40^{\circ}$ & $\begin{array}{r}6981 \\
1 \cdot 3049\end{array}$ & $6+28$ & 1.0150 & +0150 & 225 & 145 \\
\hline $50^{\circ}$ & $\begin{array}{r}8727 \\
1.4795\end{array}$ & 7660 & 9658 & -0342 & 1170 & $89^{6}$ \\
\hline $60^{\circ}$ & $\begin{array}{l}1.0472 \\
1 \cdot 6540\end{array}$ & $\cdot 8660$ & 9550 & -0450 & 2025 & 1754 \\
\hline $70^{\circ}$ & $\begin{array}{l}1.2217 \\
\text { I. } 8285\end{array}$ & '9397 & $972 \delta$ & $-{ }^{\circ} 0372$ & $13^{8}+$ & 1300 \\
\hline $80^{\circ}$ & $\begin{array}{l}1 \cdot 3963 \\
2 \cdot 0031\end{array}$ & 9848 & 1.0170 & +0170 & 289 & 285 \\
\hline $90^{\circ}$ & $\begin{array}{l}1.5708 \\
2 \cdot 1776\end{array}$ & I '0000 & 1.0888 & + os8s & 7885 & 7885 \\
\hline
\end{tabular}

We thus find scale errors practically identical with those of the absolute Minimum Error Projection of this class, and a mean square scale error greater in the ratio $\sqrt{ } 16_{4}: \sqrt{1} 5^{6}$ only, and this for a zone map of a very large extent. The following rather disparaging remark of Germain (p. i 86) is therefore not justified: "Quelle que soit la méthode adoptée, cette projection ne peut convenir pour une bande de plus de $\delta$ ou 10 degrés de latitude, et dans des limites aussi rapprochées presque toutes les projections coniques ont à peu près les mêmes arantages: quant à la question de conserver la superficie totale de la carte, elle n'est que secondaire et oblige au contraire à comprimer les superficies et les distances dans la rone moyenne, tant en longueur qu'en largeur, et à les étendre dans les dew zones extrêmes dans toutes les directions." The statement as to the compression and extension of the depth of the map is certainly not correci, an the radial scale is everywhere uniform and true.

Murdoch's I'aper was followed a month later by a letter from Mr Wim. Mountaine, F.R.S., on "A short Dissertation on Maps and (harts," in which he challenges Durdoch "to draw a map in which the superficies of 
a given zone shall be equal to the zone on the sphere, while at the same time the projection from the centre is strictly geometrical."

Murdoch replies in a Note printed as an Addendum to Mountaine's Paper giving the Rule for his Second l'rojection which is a Perspective Conical l'rojection, and he says, "But here the degrees of latitude towards the middle will fall short of their just quantity and at the extremities exceed it : which hurts the eye." He then indicates the Rule for

\section{Murdoch's Third Projection.}

In this he takes $a+\chi=\tan \chi . \delta \cot \delta$. This value of $a+\chi$ is quoted by both Germain and Tissot, but it does not appear to have been noticed that the condition of equality of total area exacts a corresponding change in the value of $n$, vis.,

$$
\begin{aligned}
& n=\frac{\sin \delta}{\delta} \cdot \frac{\sin \chi}{a+\chi} \frac{\cos \chi \sin \delta}{\delta^{2} \cot \delta}=\cos \chi\left(1+\frac{\delta^{2}}{6}+\frac{31 \delta^{4}}{360} \ldots\right) \\
& a+x \tan \chi\left(1-\frac{\delta^{2}}{3}-\begin{array}{c}
\delta^{4} \\
+5
\end{array}\right) .
\end{aligned}
$$

Scale at mid-paralle $=1-\frac{\delta^{\prime \prime}}{6}+\frac{\delta^{4}}{120} \ldots ; M=\frac{2 \delta^{5}}{45} \sin \chi$ as before. Applying these results to the same map as before we get $n=0.5265, a=0.645 \mathrm{I}$, and our lable stands as under:

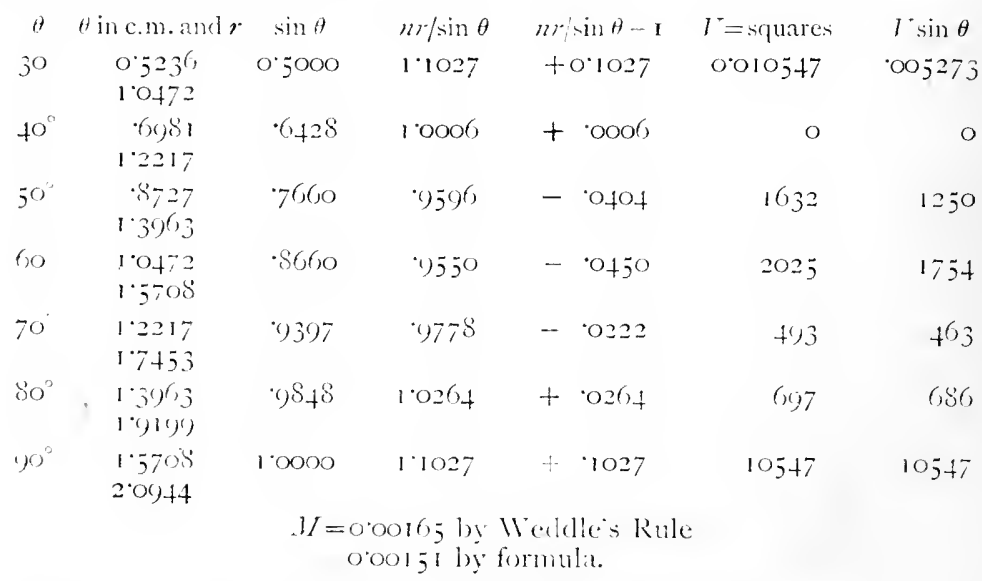

We thus have a map which has the following properties:

(i) The total area is true.

(ii) The radial scite is corrywhere true.

(iii) 'T'wo prarallels are true to scale, distant about sth depth from edeses.

(iv) The scate errors at extreme parallels are equal and about clouble the scale error of opposite sign at misl-patrallel.

(v) The arerase wate error is to first approximation the same as in the Minimum Eroor l'rojection of this class. 
(vi) The maximum scale error is less by ${ }_{10}^{1}$ th than the same quantity in the latter.

By substituting the values of $n$ and $a$ we find

$$
\begin{aligned}
\text { Scale at extreme parallels } & =\tan \delta / \delta=\mathbf{I}+\frac{\delta^{2}}{3}+\frac{2 \delta^{4}}{15} \ldots, \\
, \quad \text { mid-parallel } \quad & =\sin \delta / \delta=\mathbf{I}-\frac{\delta^{\prime 2}}{6}+\frac{\delta^{4}}{120} \ldots .
\end{aligned}
$$

This Third Projection of Murdoch therefore would appear to be on the whole the very best of the Conical Class, better for all practical purposes than the absolute Ninimum Frror, as the reduction in the maximum error more than compensates for the slight increase in average crror. It is very easily computed whether by the full formulae or by the expansions in terms of $\delta^{2}$. 'This is a curious reflection on (iemain's remark, p. I89, "Ce troisième système étant, sous tous les rapports, inférieur aux deux premiers, nous ne nous y arrêterons pas." It is also some satisfaction that it was invented by a fellow-countryman, who though born and educated in Scotland, was Rector of Stradishall, Suffolk, England.

\section{Equidistant Conical Projection with total area true.}

As in the Zenithal System the condition that the total area of the map shall be true introduces practically no difference. It affects the value of $M$ only in the third term of its expansion, and for $n$ we have the equation

whence

$$
2 \pi \int_{0}^{\beta} n \theta d \theta=2 \pi(1-\cos \beta)
$$

$$
n=2(1-\cos \beta) \beta^{2}=4 \sin ^{2} \frac{1}{2} \beta \beta^{2}-1-\frac{\beta^{2}}{12}+\frac{\beta^{4}}{360}-\ldots,
$$

differing in the third term only.

Substituting this in the expression for $L$ we find

$$
M=\mathrm{I}-\cos \beta-n \beta^{2}+n^{2} \int_{0}^{\beta} A^{2} \operatorname{cosec} \theta d \theta=\frac{\beta^{2}}{86+}\left(1-\beta_{60}^{\prime \prime} \ldots\right)
$$

as before.

The maximum scale error at the boundary

$$
=n \beta \sin \beta-2 \tan \frac{1}{2} \beta \beta=1+\frac{\beta^{2}}{{ }_{1} 2}+\frac{\beta^{4}}{I_{2}} \cdots
$$

as against

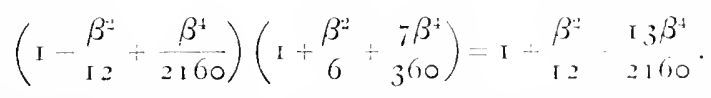

The following 'Table exhibits the map of the hemisphere in this system. in which $n=0.797$ : 


\begin{tabular}{|c|c|c|c|c|c|c|}
\hline $\begin{array}{l}\theta \\
0^{\circ}\end{array}$ & 0.0000 & $\begin{array}{c}\sin \theta \\
0^{\circ} 0000\end{array}$ & $\begin{array}{l}n \boldsymbol{r} / \sin \theta \\
0.7070\end{array}$ & $\mathrm{nr} / \sin \theta-1$ & $V=$ Squares & $V \sin \theta$ \\
\hline $15^{\circ}$ & .2618 & 25588 & .8062 & -.1938 & .03756 & 972 \\
\hline $30^{2}$ & 5236 & 5000 & .8346 & -.1654 & $.0273^{6}$ & ${ }_{13} 68$ \\
\hline $45^{\circ}$ & 7854 & 7071 & 8852 & $-11+8$ & 01318 & 932 \\
\hline $60^{\circ}$ & $1.0+72$ & $\cdot 8660$ & 9639 & -0361 & 00130 & 113 \\
\hline $75^{\circ}$ & 1.3090 & 9659 & 1.0801 & +.0801 & $.006+2$ & 620 \\
\hline $90^{\circ}$ & 1.5708 & I '000O & 1.2519 & +.2519 & .06345 & 6345 \\
\hline
\end{tabular}

In the Minimum Error Projection of this class the value of $M$ was 0.0091 and the value of the scale error at $\beta=90^{\circ}$ was +0.1835 , so the merits of the uniform radial scale are not so near those of the absolute minimum, in so large a map as a hemisphere, in this class as in the Zenithal. It is still, however, much superior to Airy's for the hemisphere, in which $M I$ was 0.0391 and the scale error at the boundary was $0^{\circ} 386_{3}$.

It may be objected to this fan-shaped Conical system that it makes a rather ugly map when $n$ is greater than say $\frac{1}{2}$, as there is a wedge-shaped gap $=(1-n) 360^{\circ}$ not filled up. This gap may, however, be filled up by continuing the map, which has the advantage of placing in juxtaposition those parts which abut on the boundaries, so that continuous measurements may be taken across those regions. 'Iheoretically speaking this advantage holds however small the limiting radius $\beta$ of the map, but it is doubtful if there would be any advantage when the gap is less than $30^{\circ}$, or $\beta$ less than about $60^{\circ}$, for all bearings from the centre are too small by about onetwelfth and, when the projection is so near a complete circle, this might be inconvenient.

\section{Plate-Carrée and Mercator Projections with total area true.}

In these cases we have $A \beta=\int_{0}^{\beta} \cos \theta d \theta=\sin \beta$ and therefore

$$
A=\sin \beta / \beta=\mathrm{I}-\frac{\beta^{2}}{6}+\frac{\beta^{4}}{120} \ldots
$$

as against

$$
1 \frac{\beta^{i j}}{6}-\frac{\beta}{72} \cdots
$$

in the absolute minimum system. It will be found that the value of $1 /$ is not altered in the first two terms of its expansion.

\section{Expansion of General Formulae in case of Zone Map.}

In the case of the \%onc Mal the liniting raclii are the same, whether the maj be (orthomorphes approximately Engal Area, or the mean of the two. vis..

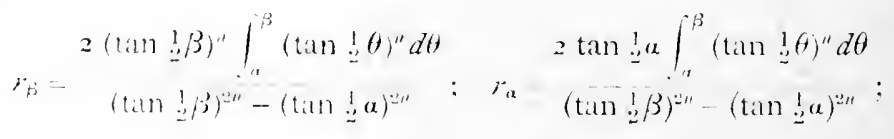


Depth of map $=2 \int_{\alpha}^{\beta}\left(\tan \frac{1}{2} \theta\right)^{n} d \theta\left\{\begin{array}{l}\left(\tan \frac{1}{2} \beta\right)^{n}-\left(\tan \frac{1}{2} \alpha\right)^{n} \\ \left(\tan \frac{1}{2} \beta\right)^{m n}-\left(\tan \frac{1}{2} \alpha\right)^{2 n}\end{array}\right\}=r_{\beta} \quad r_{\alpha}$.

Now we have found

$$
\begin{aligned}
\int_{a}^{\beta}\left(\tan \frac{1}{2} \theta\right)^{n} d \theta=2 \delta\left(\tan \frac{1}{2} \chi\right)^{n}\left\{1+\frac{\delta^{2}}{6} n(n-\cos \chi)\right. & \sin ^{2} \chi
\end{aligned}
$$

and

We have also found that

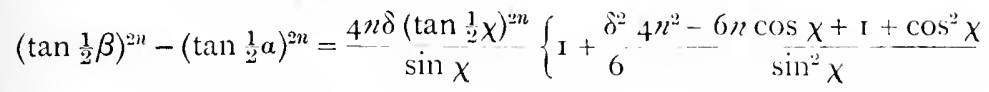

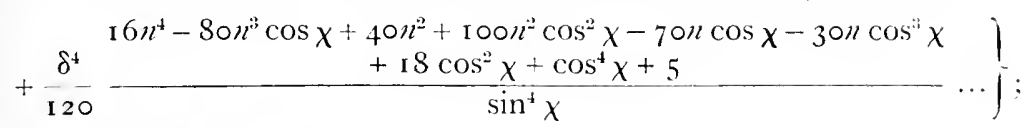

and replacing $2 n$ by $n$ we find

$$
\begin{aligned}
& \left(\tan \frac{1}{2} \beta\right)^{n}-\left(\tan \frac{1}{2} \alpha\right)^{n}=\frac{2 n \delta\left(\tan { }_{2}^{1} \chi\right)^{n}}{\sin \chi}\left\{1+\frac{\delta^{2} n^{2}-3 n \cos \chi+1+\cos ^{2} \chi}{6} \frac{\sin ^{2} \chi}{n^{2} \chi}\right. \\
& \left.+\frac{\delta^{4}}{120} \frac{n^{4}-10 n^{3} \cos \chi+10 n^{2}+25 n^{2} \cos ^{2} \chi-35 n \cos \chi-15 n \cos ^{3} \chi}{+18 \cos ^{2} \chi+\cos ^{4} \chi+5}-\ldots\right\} \text {. }
\end{aligned}
$$

Dividing out we find

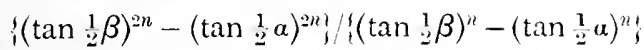

$$
\begin{aligned}
& =\frac{\left(\cot \frac{1}{2} \chi\right)^{n}}{2}\left\{\mathrm{r}-\frac{\delta^{2}}{6} \frac{3 n^{2}-3 n \cos \chi}{\sin ^{2} \chi}\right. \\
& \left.+\frac{\delta^{4}}{360} \frac{75 n^{4}-90 n^{3} \cos \chi-60 n^{2}-15^{n^{2}} \cos ^{2} \chi+75^{n} \cos x+15^{n} \cos ^{3} x}{\sin ^{4} \chi} \ldots\right\} \\
& =\frac{\left(\cot \frac{1}{2} \chi\right)^{n}}{2}\left\{\mathrm{I}-\frac{\delta^{4}}{60} \cot ^{2} \chi+\frac{\delta^{4} \cot ^{2} \chi}{2 t}\right\} \\
& =\frac{\left(\cot \frac{1}{2} \chi\right)^{n}}{2}\left\{\mathrm{I}+\frac{\left.\delta^{4} \cot ^{2} \chi\right\}}{40}\right\} \text { if } n=\cos \chi\left(1+\frac{\delta^{2}}{30}\right)
\end{aligned}
$$

and

$$
=\frac{(\cot 2 x)^{n}}{2}\left\{1+\frac{\delta^{4} \cot ^{\prime \prime} \chi}{24}\right\} \text { if } n-\cos x \operatorname{simply}
$$

Therefore

$$
r_{\beta}-r_{a}=2 \delta\left(1+\begin{array}{c}
\delta^{4} \cot ^{2} x \\
4.5
\end{array}\right) \text { if } n \cos x\left(1+\frac{\hbar^{2}}{30}\right)
$$

and

$$
=2 \delta\left(\begin{array}{c}
1+\delta^{\prime} \cot ^{\prime \prime} x \\
3^{\circ}
\end{array}\right) \text { if } n=\cos x \operatorname{simply.}
$$

'These shew how nearly the depth of the may) is true. 
We shall now obtain an expression for the radius of the mid-parallel and it will be sinipler to treat the orthomorphic part separately, where

$$
\begin{aligned}
& r=2 A\left(\tan \frac{1}{2} \theta\right)^{n}=\left[2 \int_{\alpha}^{\beta}\left(\tan \frac{1}{2} \theta\right)^{n} d \theta /\left\{\left(\tan \frac{1}{2} \beta\right)^{2 n}-\left(\tan \prod_{2}^{1} \alpha\right)^{2 n\}}\right]\left(\tan \prod_{2}^{1} \theta\right)^{n}\right. \\
& =\text { (using above expansions) }
\end{aligned}
$$

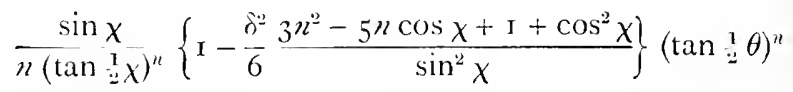

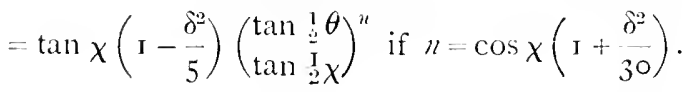

$$
\begin{aligned}
& \text { Thus when } \theta=\chi, \quad \quad r_{\lambda}=\tan \chi\left(\mathrm{I}-\frac{\delta^{2}}{5}\right) \text {, }
\end{aligned}
$$

the same as in the Equidistant System.

Now put $\theta=\chi+\epsilon$, where $\epsilon$ is the angle from mid-parallel to any other; then

$$
\begin{aligned}
& \left(\tan \prod_{z}^{1} \theta\right)^{\prime \prime}=\left(\tan \frac{1}{2}(x+\epsilon)\right)^{n}
\end{aligned}
$$

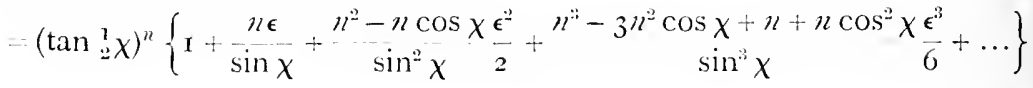

$$
\begin{aligned}
& (\tan ! \cdot \chi)^{n}\left\{\mathrm{I}+\epsilon \cot \chi+\epsilon \cot \chi \frac{\delta^{2}}{3^{\circ}}+\frac{\epsilon^{\prime \prime}}{6} \cot \chi \cdots\right\} \text { if } n=\cos \chi\left(\mathrm{I}+\frac{\delta^{2}}{3^{\circ}}\right),
\end{aligned}
$$

therefore

$$
r_{\epsilon}-r_{X}=\left(\mathrm{I}-\frac{\delta^{2}}{5}\right) \epsilon\left(\mathrm{I}+\frac{\delta^{2}}{30}+\frac{\epsilon^{2}}{6} \cdots\right) \epsilon\left(\mathrm{I}-\frac{\delta^{2}}{6}+\frac{\epsilon^{2}}{6} \cdots\right) .
$$

This gives a very ready method of calculating and plotting the Minimum Error Orthomorphic Projection of a zone, when once the radius of the midparallel has been computed.

In the approximate Equal Area Projection

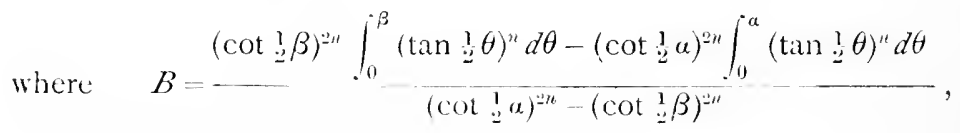

we shall find for the radius of mid-parallel

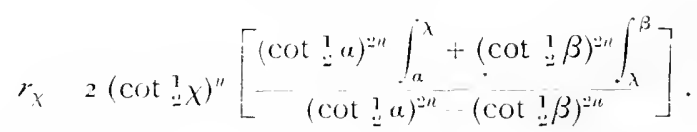

Expanding this expression in the same way we shall find

$$
r_{x} \sin _{n} \chi\left(1-\frac{\delta^{2}}{6}\right)-\tan x\left(\begin{array}{ll}
\delta^{2} \\
5
\end{array}\right) \text { if } n=\cos \chi\left(1+\begin{array}{c}
\delta^{\prime \prime} \\
30
\end{array}\right)
$$

and coaluatiog $r_{e}-r_{x}$ in the same way we get $\epsilon\left(1+\frac{\hat{n}^{2}}{6}-\frac{\epsilon^{2}}{6}\right)$; so that the mean of this and the orthomorphic part is simply $r_{t}-r_{t} \epsilon$, the Equidistant value. 


\section{The Albers Equal Area Projection.}

The Projection we have just considered is not strictly Equal Area since it satisfies the differential equation $d r / d \theta-\mathbf{I}=-(n r / \sin \theta-1)$, whereas the strictly Equal Area Projection satisfies the diffurential equation

$$
d r / d \theta-\sin \theta / n r .
$$

The complete solution of the latter is

$$
r=\left(r_{i}^{2}+\frac{4}{n} \sin _{2}^{2}: \theta\right)^{\frac{1}{2}}
$$

and is called the Albers Projection. When the Projection extends to the centre $r=0$ when $\theta=0$, and $r=2 \sin ? \theta_{i} \sqrt{ } n$ simply. We shall now investigate what value of $n$ makes the mean square scale error a minimum. Putting $\sqrt{ } n=\mathrm{I}+a$, so that $\frac{\mathrm{I}}{\sqrt{ } n}=\mathrm{I}-a+a^{2}-\ldots$, we have

$$
\begin{gathered}
r=\left(\mathrm{I}-a+a^{2} \ldots\right)\left(\theta-\frac{\theta^{3}}{24}+\frac{\theta^{3}}{1920} \ldots\right), \\
d r / d \theta=\mathrm{I}-\frac{\theta^{2}}{8}+\frac{\theta^{4}}{38^{4}}-\ldots-a(\mathrm{I}-a)\left(\mathrm{I}-\frac{\theta^{2}}{8} \ldots\right), \\
n r / \sin \theta=\mathrm{I}+\frac{\theta^{2}}{8}+\frac{5^{4}}{384}+\ldots+a\left(\mathrm{I}+\frac{\theta^{2}}{8} \ldots\right), \\
M=\int_{0}^{\beta}\left\{(d r / d \theta-\mathrm{I})^{2}+(r / \sin \theta-\mathrm{I})^{2 !}\right\} \sin \theta d d \theta \\
=\int_{0}^{\beta}\left\{\frac{\theta^{4}}{6+}\left(\mathrm{I}-\frac{\theta^{2}}{48}\right)^{2}+\frac{a \theta^{2}}{4}(\mathrm{I}-a)\left(\mathrm{I}-\frac{\theta^{2}}{48}\right)\left(\mathrm{I}-\frac{\theta^{2}}{8}\right) \ldots+a^{2}\left(\mathrm{I}-2 a^{2}\right)\left(\mathrm{I}-\frac{\theta^{2}}{8}\right)^{2}\right. \\
\left.+\frac{\theta^{4}}{64}\left(\mathrm{I}+\frac{5^{2}}{48}\right)^{2}+\frac{a \theta^{2}}{4}\left(\mathrm{I}+\frac{5^{2}}{48}\right)\left(\mathrm{I}+\frac{\theta^{2}}{8}\right) \ldots+a^{2}\left(\mathrm{I}+\frac{\theta^{2}}{8}\right)^{2}\right\} \sin \theta \cdot d \theta,
\end{gathered}
$$

whence for a mininum we shall find

or

$$
a=-\frac{\beta^{2}}{16}+\frac{\beta^{4}}{512} \ldots
$$$$
\mathrm{I}+a=1-\frac{\beta^{2}}{\mathrm{I} 6}+\frac{\beta^{4}}{5 \mathrm{I} 2},
$$

and

$$
(\mathrm{I}+a)^{2}=n-\mathrm{I}-\frac{\beta^{2}}{8}+\frac{\beta^{4}}{\mathrm{I} 28},
$$

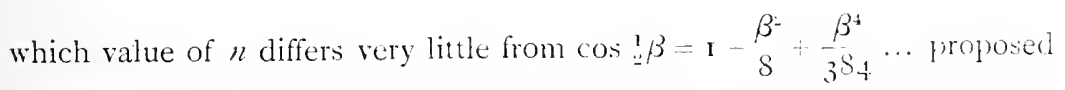
by II. Tissot to make the maximum scale error as smatl ins pussible. The limiting radius $\gamma_{\beta}=\beta\left(1+\frac{\beta^{2}}{48}-\frac{\beta^{4}}{7680} \cdots\right)$ and the maximum scale crror thereat $=n r_{\beta}, \sin \beta=\mathrm{I}+\frac{\beta^{2}}{16}+\frac{11 \beta^{4}}{1536}$ which differ by small amounts in the third terms found for the approximate Eupual Area Minimum Lrror system. The value for $M$ is $\frac{\beta^{\text {hi }}}{70 \bar{s}}$ to the first term, the same as in the latter systeml. 
As to the Albers Projection for a zone, $r=\left(r_{0}{ }^{2}+\frac{4}{n} \sin ^{2} \frac{1}{2} \theta\right)^{\frac{1}{2}}$, the writer has not succeeded in deriving by rigorous mathematical processes the values of the constants $r_{0}$ and $n$ which render its mean square scale error a minimum, the requisite integrations and expansions being very complex. By writing the formula in the form $r=\{a(c-\cos \theta)\}$, where $a=2 / n$ and $a(c-\mathrm{I})=r_{i 1}^{2}$, we have

$$
d r / d \theta=a \sin \theta / 2\{a(c-\cos \theta)\}^{\frac{1}{2}}, \quad n r / \sin \theta=2\{a(c-\cos \theta)\}^{\frac{1}{2}} / a \sin \theta,
$$

and

$$
M=\int_{\alpha}^{\beta}\left\{\frac{a \sin ^{3} \theta}{4(c-\cos \theta)}-\underset{(c-\cos \theta)^{\frac{1}{2}}}{\sqrt{ } a \sin ^{2} \theta}+\begin{array}{c}
4(c-\cos \theta) \\
a \sin \theta
\end{array}-\frac{4(c-\cos \theta)^{\frac{1}{2}}}{\sqrt{ } a}+2 \sin \theta\right\} d \theta \text {. }
$$

We can now expand the integrals in terms of the middle value $\chi=\frac{1}{2}(\beta+\alpha)$ and the limits $\delta=\frac{1}{2}(\beta-\alpha)$, and we may put in the expansions

$$
a=2 / n=2 / \cos \chi(\mathrm{I}+e), \quad c-\cos \chi=\sin ^{2} \chi(\mathrm{I}+f) 2 \cos \chi,
$$

where $e$ and $f$ are corrections depending on $\delta^{*}$ and higher terms, the values of which are to be found so as to make $M$ a minimum. The writer has not taken the expansions beyond $\delta^{*}$ and so far he finds the equation $c+f+r_{: 5}^{1} \delta^{\prime \prime}=0$ must be satisfied.

By analogy with the approximate Equal Area function, we may however assume that if we make the scales at upper and lower limits equal and the total depth of the map true, we shall not be far from the absolute minimum mean square error conditions. These assumptions have been worked out by Fiorini (p. 269) and amount to taking

$$
\begin{array}{rc}
C=\frac{\mathbf{I}+\cos \alpha \cos \beta,}{2 \cos \chi \cos \delta}, a & \begin{array}{c}
2 \delta^{\prime \prime} \cot \delta \\
\cos \chi \sin \delta
\end{array} \\
C-\cos \chi=\frac{\sin ^{2} \chi\left(1-\frac{\delta^{\prime \prime}}{2}\right)}{2 \cos \chi}, & a=\frac{2\left(1-\frac{\delta^{2}}{6}\right)}{\cos \chi}:
\end{array}
$$

thus $e=+\frac{\delta^{2}}{6}, f=-\frac{\delta^{2}}{2}$ and our equation $e+f+\frac{1}{3 ;} \delta^{2}=0$ is satisfied. We also find

$$
n=\cos \chi\left(1+\frac{\delta^{*}}{6}\right), \quad r_{\chi}=\tan \chi\left(1-\frac{\delta^{\prime \prime}}{3}\right),
$$

the same values as in Murdoch's 'Third Projection.

To exemplify this result we shall compute the same map from $\alpha=30^{\circ}$ to $\beta=90^{\circ}$ (Upper table). We find $n=0.5265, a=37988, C=I^{\circ} I_{5}+7$.

Tissot (p). 222-6) has investigated the values of the constants of the Albers l'rojection to make the maximum scale crror as small as possible. 'This involves making the sciles at upper and lower parallels equal and also exptal in value to the maximum scale of opposite sign which oceurs near the mid-parallel. We shall now compute the sime map on 'Tissot's assumption, for comparison (lower table). In his notation $r=\left(n_{10}+\frac{4}{11} \sin ^{2} \cdot 2 \theta\right)^{\frac{1}{2}}$, 


\begin{tabular}{|c|c|c|c|c|c|c|c|c|c|}
\hline$\theta$ & $\begin{array}{c}\cos \theta \\
C-\cos \theta\end{array}$ & $a(C-\cos \theta)$ & 3) $r$ & $m r / \sin \theta$ & $d r / a \theta$ & $m / \sin \theta-1$ & $(d) \cdot d \theta-\mathrm{t}$ & $\begin{array}{l}\text { Squares } \\
\text { and } V\end{array}$ & $V \sin \theta$ \\
\hline $30^{\circ}$ & $\begin{array}{r}0.8660 \\
\cdot 2887\end{array}$ & $1 \circ 097$ & 1.048 & $1 \cdot 1 O_{4}$ & 0.906 & $+\cdot 104$ & - .094 & $\begin{array}{r}0110 \\
88\end{array}$ & .0O() \\
\hline $40^{\circ}$ & $\begin{array}{l}7660 \\
\cdot 3887\end{array}$ & 1477 & $1 \cdot 215$ & 995 & 1.005 & -.005 & $+\mathrm{OO}_{5}$ & $\begin{array}{r}0198 \\
0000 \\
0\end{array}$ & $0 \times 100$ \\
\hline $50^{\circ}$ & $\begin{array}{l}6+28 \\
5119\end{array}$ & 1945 & 1.395 & 959 & 1.043 & $-{ }^{\circ} \mathrm{O}+\mathrm{I}$ & $+0+3$ & $\begin{array}{r}.0000 \\
.0016 \\
18\end{array}$ & .0026 \\
\hline $60^{\circ}$ & $\begin{array}{l}5000 \\
65+7\end{array}$ & 2487 & 1.577 & 959 & 1.043 & $-0+1$ & $+{ }^{\circ} \mathrm{O}+3$ & $\begin{array}{r}.0034 \\
.0016 \\
18\end{array}$ & .0029 \\
\hline $70^{\circ}$ & $\begin{array}{l}3+20 \\
\cdot 8127\end{array}$ & 3.087 & 1757 & $98_{4}$ & 1016 & -016 & +.016 & $\begin{array}{r}.0034 \\
.0003 \\
3 \\
.0006\end{array}$ & .0006 \\
\hline 80 & $\begin{array}{l}1736 \\
9811\end{array}$ & 3727 & 1.931 & 1032 & 960 & +032 & $-0_{3} \mathrm{I}$ & $\begin{array}{r}.0010 \\
10\end{array}$ & 0020 \\
\hline \multirow{3}{*}{$90^{\prime \prime}$} & & & & & & & & 0020 & \\
\hline & $\begin{array}{r}0000 \\
1 \cdot 1547\end{array}$ & 4386 & 2.094 & $1 \cdot 102$ & 907 & $+\cdot 102$ & -093 & $\begin{array}{r}.0104 \\
86\end{array}$ & .0150 \\
\hline & & & & & & & & .0190 & \\
\hline
\end{tabular}

By Weddle's Rule $. M=0^{\circ} 003 \mathrm{I}$ as against $0^{\circ} 00300$ for double Murdoch's Third Projection and $\mu=0^{\circ} 060$, so we may be sure we are very close to the conditions for absolute minimum of $M$.

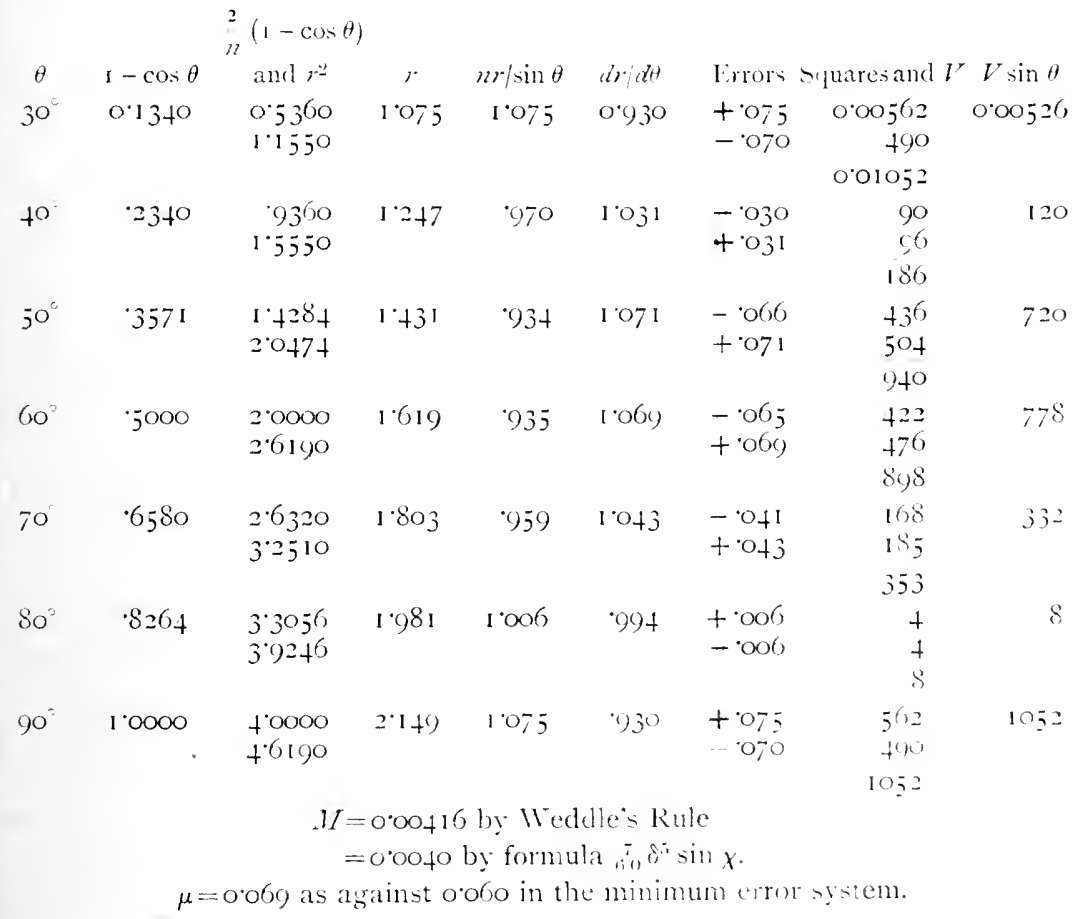


$H=$ maximum positive scale value near mid-parallel, $\delta=$ co-latitude of that parallel of maximum scale error, then if

$$
\begin{gathered}
\delta=\frac{1}{2}(\beta-\alpha), \quad H=(\sec \delta)^{\frac{1}{2}}=1 \cdot 075, \quad n=\cos \chi=\frac{1}{2}, \quad \cos \delta_{\mu}=n H^{2}=0.5773, \\
\delta_{\mu}=54^{\circ} 44^{\prime} ; \quad r_{0}=(1-\cos \delta \mu) / n H=0.7868, \quad r_{01}{ }^{2}=0.6190 .
\end{gathered}
$$

The maxinuum scale error has been reduced from $10 \cdot 3$ per cent. to 7.5 per cent. at the expense of increasing the mean square scale error from 6.0 per cent. to 6.9 per cent. It will be olsserved however that this maximum error occurs at the very edges of the map and then rapidly diminishes, whereas in 'Tissot's system the errors between the standard parallels are much larger, being $50 \%$ greater at the mid-parallel.

When the mid-parallel in Albers' Projection becomes the Equator the formula changes into $r-r_{0}=\frac{\mathbf{I}}{n^{\prime}} \sin \phi$, where $\phi=90^{\circ}-\theta$ or angle from equator. The minimum value of $M$ will be found by taking

$$
n^{\prime}=\sin \delta / \delta=\mathrm{I}-\frac{\delta^{2}}{6}+\ldots
$$

\section{Map of the World in three parts in Minimum Error Conical System as proposed by $\mathbf{M}$. Collignon.}

According to Germain (p. เ 03 ) M. Collignon proposed to construct a map of the World in three parts by projecting the portion of the surface from the poles to latitude $30^{\circ}$ on the Isospheric Stenoteric System of Lambert, i.e. the Conical Equal Area System or $r=2 \sin \frac{1}{2} \theta / \sqrt{ } n$, and the middle zone covering $30^{\circ}$ on each side of the Equator on the Isocylindric System. The greatest error of distance is said to be 15 per cent. and of angle $8^{\circ}$ i $2^{\prime}$.

If we use the Minimum Error Equidistant Conical System instead, we have for the parallel scale error at $60^{\circ}$ from the pole

$$
+\beta^{2} / 12=\left(\text { c.m. of } 60^{\circ}\right)^{2} / 12=9 \text { per cent. }
$$

and for the middle part we have in Murdoch's Third P'rojection parallel scale error at $30^{\circ}$ from Equator $=+0^{2} / 3=\left(\mathrm{c} . \mathrm{m} \text {. of } 30^{\circ}\right)^{\circ} 3$, i.e. the same, so that the sections would have a rolling fit along the boundaries. The greatest alteration of angle would be $2 \sin ^{-1} \frac{0.09}{2.09}$ : about $5^{\circ}$. The mean square crror in the conical parts $=\mathrm{I}-\beta_{12}^{2}=0.9 \mathrm{I}$.

This is probaluly the lest map of the World that can be obtained in three separate shects. The midelle portion of the map covers exactly half the total surface of the splere and the end portions each one quarter. 'The scale aleing the lifuator is of course too small by half the excess at latitude $30^{\circ}$, or ly abrut t! per rent. 


\section{G. W. Hill's Projection.}

The celebrated American astronomer and mathematician, (i. II. Hill, in a I'aper published in the Annals of Mathematics, Second Series, Vol. 10 , No. I, October 1908, investigates a projection which he claims to be the best possible on Tchebycheff's (or Chebyehev's) P'rinciple that " $A$ map is the best when, within its limits, the logarithm of the seale has the least possible oscillation." This Paper is rather difficult, but it appears to be stipulated that the map shall be conformal, i.e. orthomorphic, and the principle leads to the result that the seale error must vanish all round the boundary.

The scale along the parallels is no longer uniform and the meridians in addition to having a variable scale are no longer straight lines

Hill applies his results to an example extending from latitude $f^{\circ}$ to latitude $50^{\circ}$ and for a difference of longitude extending $5^{\circ}$ on eatch side of a central meridian. He gives a table of scale errors of which the sum is 0.0754 for 66 values, the arithmetic mean of which is 0.00114 and the maximum 0.00254 .

If Airy's Zenithal System had been applied to the same map it would be comprised in a radius $\beta$ of about $6^{\circ} \cdot 3$, and so the mean squilre scale error $\mu$ would be $\beta^{2} / \sqrt{ } 192=0.000906$, the average error $=0.7979 \mu-0.00072$, and the maximum error $\beta^{2}, 8=0.0018$.

If the Conical System were applied to the same map a zone $10^{\circ}$ wide would cover it, the mean square scale error would be $\delta^{2} 6 \cdot 7$ (where $\delta-\mathrm{cm}$. of $\left.5^{\circ}\right)=0.00 \mathrm{I}$, and the maximum error would be $0^{\circ \prime}, 3=0.0025$.

It does not appear that Hill's Projection has any advantage over those we have investigated and it is certainly more difficult to compute.

It still remains an open question, perhaps, as to whether there is a class of projections, i.e. of developable surfaces, better than the Conical. The writer's impression is that there is not, but so far he has not been able to prove this absolutely.

\section{Best projection for a given country.}

As we have already stated, the best projection for a given country depends on the shape of the country. Thissot has developed a method of finding such a projection on the principle of making the maximum scale error as small as possible. He applies this to finding the hert projection for a map of spain and $(p, 69)$ he gives the resulting scale errors. The map extends from latitude $36^{\circ} 0^{\prime}$ to latitude $4.350^{\prime}$. The replesentition appears to be nearly conformal. The maximum seale error in 0.00119 and the mean square scale crror works ont it c.001: 6 .

If the Zenithal System had been usecl it alpears that spain could be included in a radius $\beta$ of about $5^{\circ}=0.08 ; \mathrm{cm}$. If the biquitistunt limimum Error Projection were used the maximum scale error would be $\beta=8-0.000,5$. and the mean square scale crror would be $\beta^{2} \cdot \mathrm{I}^{\prime} \mathrm{I} \quad 0.00058$. 'This is there- 
fore better than Tissot's, for we could have conformality with the same maximum error and a mean square error of $\beta^{2} / \sqrt{ } / 96=0.00077$.

If however Murdoch's Third Projection were used we could take $\beta-\alpha=8^{\circ}$ and $\delta=4^{\circ}=0.070 \mathrm{~cm}$. Hence the maximum scale error would be $\delta^{2 / 3}=0.0016$ and the mean square scale error would be $\delta^{2} / \sqrt{ } 45=0.00073$. This could be made conformal like Tissot's with the same maximum error and by increasing the mean square error to $0.00073 \times \sqrt{2}=0.00102$.

Either of these projections is therefore at least as good as 'Tissot's and both are easier to work out.

\section{Best projection for Italy.}

Mr J. I. Craig, of the Egyptian Suriey 1)epartment, in his pamphtet on Map Projections, raises the question of the best projection for Italy, a long narrow country with a gencral bearing of about $45^{\circ}$. It would appear that a zone about $5:^{\prime c}$ wide would cover Italy proper, excluding Sicily. If we used an oblique conical system we could represent Italy on Murdoch's Third Projection with a maximum scale error of $(0.0 .48)^{*} / 3=0.00077$ and a mean square scale error of $0.00230 / 13^{1} \mathbf{r}=0.00017$. 'To represent the same country on the Equidistant Zenithal System we should have to take $\beta=$ about 5.:.$^{\circ}$ and then the maximum error would be $\beta^{2} / S=0.001$ I 5 and the mean sequare error wonld be $\beta^{2}, 13 \cdot 1=0.00070$.

It appears therefore that we shall only do better in the Zenithal System, for countries of moderate extent, if $\beta^{*} / I_{3} \cdot 1$ is smaller than $\delta^{2}, 6 \%$, that is if $\beta: \delta$ is less than $\mathrm{r}+\mathrm{r}$, or if the comntry to be represented can be included in a parallelogram whose longer to shorter side is less than about $r+$ to $r$. It must be remembered that the Conical is always, theoretically speaking, better than the \%enithal System, but we are excluding the conical projection up to pole of projection, where such pole would occur in the middle of a country of moderate size and produce a fan-shaped map with a small gap $=360^{\circ}(1-n)=15^{\circ} \beta^{\prime 2}$.

As we have remarked Hammer has published Tables for Conical Projections with pole in any position. The formulae are capable of a little simplification when the axis is inclined at $45^{\circ}$ to the meridian as in Italy, but even then this skew system cannot be recommended to cartographers, as the decrease of scale errors will be more than counter-balanced by the disalvantages of complex formulac and contrary flexure of the projected meridians and parallels.

\section{Summary of Results.}

The Minimun Enror Projections of (ieneral Conical Class have been thoroughly examined by investigating mathematically the general function an found by the calculus of Vartations.

As in the \%nithal Class it hats been found possible to simplify this formula by capresion and by the stipulation of making the total area of 
the map true, with no increment in the mean sfuare error to the first or even second term of its expansion.

The Minimum Error Conical l'rojection of a zonc is then found to be identical with the Third Projection of Murroch invented in 1758 .

This can be made Orthomorphic or Equal Area as may be desired, with no change in the depth of map or ratlus of midl-parithel, by small and easily calculated changes in the radii of the intermediate parallels, thus producing two standard parallels at about one-fifth the depth of the map from the limiting parallels.

This class includes the Cylindrical Projections, Direct and Transverse, in which the property of Minimum Error will also be produced by making the depth of the map true.

When the Conical Projections extend up to the pole the principle of making the total area true also applies, but the Equidistant I'rojection is not quite so near the absolute Minimum Error as in the zone map. It is however still recommended as the best in practice on account of its simplicity.

The general practice hitherto has been to make the maximum error in this class as small as possible. This practice leads to a maximum error one-twelfth smaller than in the system now investigated, but the arerage error is $\mathrm{r}_{5}$ per cent. greater, and the valuable property of truth of radial scale and total area is lost.

\section{The best projection for a Cadastral Survey.}

A good many of the refinements we have been considering may appear unimportant and unnecessary to the practical map-maker, so long as his work must be plotted on a substance like paper which changes owing to hygrometric conditions in a way that cannot be allowed for, from dily to day, and even from hour to hour. It is prudent however to carry our refinements to a degree further than the limits of such changes, and we should always look forward to the possibility of some substance less changuable than paper, as we know it, being invented.

But there is one case in which the greatest care in sulecting the besi projection should be exercised and that is the projection on which to base the rectangular plane coordinates of a cadastral survey.

In the great self-governing Colonies and in most of the Crown colonies the system of land tenure is based on accurate survey. loundaries of pro. perty are defined by the bearing and distance of lines between fiducial points on permanent marks set at intervals round the propenty. The surey round this boundary is closed to a preseribed limit of crror by the meihor? of balancing latitude and departure, and one or two points of it are connected to the general triangulation of the country either dircetly or by intermediate traverse survey to a trigonometricilly lived point. 
The Cadastral Survey Department does not want to be worried with questions of spherical excess or excentricity of the Earth, and in supplying it with a system of plane coordinates we ought to ensure that the errors of the projection chosen will lie well within the limits of accidental error allowed in the Cadastral Branch.

An illustration from the Survey on which the writer was engaged may not be out of place. This was in a tropical country for the most part undeveloped and covered with virgin forest, even up to heights of 5000 feet, consisting of huge trees from roo to nearly 200 feet high, intertwined near the ground by a dense mass of undergrowth and creepers, through which it was impossible to force a way unless preceded by a man with a clearing knife. The country was covered with a network of najor triangles by the 'Trigonometrical Branch, of sides averaging say 20 miles in length, and very much of the interlaced type used in Great Britcin and Ireland. The elearing of hill-tops and suitable points on the lower ground for this purpose involved great expenditure of money and labour. This major triangulation was however of very little direct use to the Cadastral (or, as it was called, Revenue) Branch, as settlement in such countries always takes place at the beginning along rivers, roads and railways, which are necessarily on low ground. A general system of minor triangulation was in this case ruled out by the great cost of clearing. But, owing to the development of the system of accurate traversing with the long tape of steel or invar, first instituted in Australia, the difficulty was overcome. The method used was to run high class traverses, locally called standard traverses, along roads, railways, and rivers (and sometimes watersheds), leaving permanent marks in pairs or threes every half-mile or so. The traverses were comnected with the major triangulation at intervals where suitable opportunities occurred, generally from 7 to ro miles apart, either directly or by the fixation of suitable minor points. The limit of error allowed in such standard traverses when closed on trigonometrically fixed points was I in 10,000 , but the average yearly limit attained was near $\mathrm{I}$ in 30,000 . These traverses then afforded a frame-work ready at hand in the most convenient places for the Cadastral Branch, with which to connect their boundary surveys. 'I'he limit of error allowed in the Cadastral Branch was $\mathbf{I}$ in $\$ 000$, but the average attained was more like I in 10,000.

The projection used in this survey was the same as that used in Great Britain, India, South Africa, ete, namely the Cassini or 'Transverse Cylindrical Equidi-tant, cast on an average value of the farth's radius in the vicinity, and with only one standircl (so-called) parallel, i.e. the meridian. We have seen that in this projection the $x$ or $\mathrm{N}$-s coordinates are always too large in the ratio nec $\theta: 1$, where $\theta$ is the spherical angle of a point away from the central meridian. When $\theta$ is small the error is therefore ${ }_{2}^{\prime} \theta^{2}$. If $\theta$ correspomels with a distance of 50 miles, ats it diel in one instance in this survey, this error amounts to ${ }_{2}^{1}\left(\begin{array}{c}50 \\ 69 \times 57\end{array}\right)^{2}$ I in a 2,300 nearly; which 
is rather uncomfortably near the limits of error in the traverse surveys. All areas on which land-tax is paid are increased to the same degree, which is an inconvenience to the lessee at any rate. Of course this error was allowed for in stating the closing errors of the traverse surveys, but the smaller it can be made the better, and our investigations have shewn that by reducing all N-S coordinates in the proportion ${ }_{1}^{1} \delta^{*}$, where $\delta$ is the liniting spherical angle of the projection from the central meridian, the maximum error is reduced in the ratio $2: 3$, and the total area of the projection at any rate is made correct.

It is curious that this device of making standard two parallels (or submeridians) in the Conical or Cylindrical Projections when used in national surveys, does not seem to have been noticed beforc. The only mention the writer can find of the use of such a device is by Jordan in referring to the Survey of Mecklenburg (1) 350) where the Conical Orthomorphic Projection with initially one standard parallel was used, but by a little device (durch einen kleinen Kunstgriff), which was evidently the use of a scale constant $1-\frac{\delta^{2}}{4}$, the maximum scale error was reduced one-half.

The Cadastral Branch, as stated, was supplied with the rectangular coordinates of the trigonometrically fixed points and the points fixed in the standard traverses, in this Cassini System, but in supplying hearings from triangulation stations such bearings were given from a meridian at right angles to the great circle from such station to the principal meridian, according to the practice usually followed hitherto. Thus the bearing from a point $P$ to a point $Q$ would differ from the bearing from $Q$ to $P$ by the spherical excess of the space $p P Q q$, where $f$ and $q$ are the feet of the perpendiculars from $P$ and $Q$ to the principal meridian. This differunce might amount to 13 seconds of arc for a distance $P Q \quad 20$ miles ruming parallel to the principal meridian and 50 miles east or west of it. This is a small amount compared with the accumulated error of a traverse 20 miles long, but still such differences are rather puzzling and inconvenient to the Cadastral Surveyor, and having once chosen a projection it would be better, in the writer's opinion, to supply all bearings as well as coordinates, on the plane system.

For a country nearly round or stuare, from which the triangulation is not likely to be continued in the $\mathrm{N}-\mathrm{S}$ or $\mathrm{E}-1 \mathrm{l}$ elirection for any purpose to neiglıbouring countries, the Minimum Error Equidistant \%enithal I'rojection has many advantages. As alrealy stated the maximum crror would be less than in the Cassini in the ratio $s: 3$ ant the amount of splperical excess to be forcibly dispersed would be about one-half. The projection is easily calculated for it is only necessary to calculate by spherical trigonometry the bearings and distances of all points from the ecentre of projection, and then to reduce such distances in the ratio $1-1 \beta^{\prime}: 1$. Which me.m sub tracting a constant from the log distances, betore working the concilinates by simple sine and cosine of the bearing. 


\section{Projection for Surveys of Hanover and Egypt.}

As already remarked in the surveys of Hanover and of Egypt the Cassini Projection was rejected in favour of the Conformal, or Orthomorphic, Cylindrical System of Gauss. This differs from the Cassini in the fact that, in order to produce the same scale error in the $\mathrm{E}-\mathrm{IV}$ direction as in the $\mathrm{N}-\mathrm{S}$ direction, the coordinate $y$ in the former direction instead of being the true distance on the sphere is increased in the ratio

$$
\int \sec \theta d \theta \theta\left(\mathbf{I}+{ }_{15}^{1} \theta^{2}+\dddot{1}_{1}^{1} \theta^{4} \ldots\right)
$$

to $\theta$, that is $y$ is increased to $y\left(1+\frac{1}{6} \frac{y^{2}}{R^{2}} \ldots\right)$, where $R$ is the Earth's radius in the vicinity. The projection as used in the surveys mentioned has only one standard parallel, i.e. the principal meridian. It could be improved in the same way as the Cassini by multiplying both coordinates by the scale constant $\left(\mathrm{I}-{ }_{i}^{1} \delta^{2}\right)$, where $\delta$ is the limiting spherical angle from the principal meridian, which would also make the total area correct.

In the Orthomorphic Projections the errors of bearing for small distances round a point are practically zero and for large distances are much smaller and easier to compute than in the Equidistant Projections. 'The crrors of distances are also easier to compute. For these reasons the writer is inclined to think that for Cadastral Survey purposes these advantages more than counterbalance the cxtra initial labour of computing the radial coordinates, and that Surveys about to start a new or revised system of projection would be well advised to consider the merits of the orthomorphic projections, whether direct or transverse, and to adopt the scale constant which renders the total area true and the average error a minimum. The advantages of the orthomorphic system have also become apparent during the war for artillery and aerial purposes, the new projection for the war maps of France being based on the Conical Orthomorphic System with two standard parallels, though with Tissot's criterion of least maximum error instead of the criterion of least average error as recommended in this Paper. This map being more liable to extension in the E-W direction is on the lirect system and the standard parallels are actual parallels of latitude.

\section{The Finite Errors in Conical and Cylindrical Projections.}

So far we have only discussed the differential scale errors at a point. but as Hinks has pointed out (Chapter IX) these are perhalps not of much use in practice, when the (juestion mostly arises of finding the actual errors in clistance and bearing between two points at finite distances aprart on the map), as compared with the corresponding (puantitics on the sphere or spheroid. Formulate for surb finite displacenents have been investigated to sereral terms of the ir expansions in the works of Jordan and Helmert 
for the Cassini Projection, and in Jordan for the Gauss Conformal (or Transverse Cylindrical Orthomorphic), and I irect Conical Orthomorphic Projections. Jordan also investigates the coordinates and finite errors of the Direct Cylindrical Projection, Equidistant and Orthomorphic, tangent along the prime vertical great circle at a point, and called by lim the "Querachsige l'rojektion." 'This, as he shews, only differs from the (onical Projection, tangent along a parallel of latitude, by small quantitics depending on the fourth powers of the coordinates. The latter has one advantare over the former in that the projected meridians are absolutely strainht lines. In all these investigations only one standarel parallel is assumed, but the results will apply to the case of two standard parallels without rhange for the Orthomorphic l'rojections so far as the finite angular eorrections are concerned, for the scale constant is applied to both coordinates, and so far as distances are concerned it will simply be necessary to decluet the sale constant from the distance ratio as given by the formula of Jorkan. In the case of the Equidistant Projections, Direct and Transiere, the formula for two standard parallels will become more complex as will be shewn later, but the scale constant will tend to reduce the amounts of the corrections.

\section{A mathematical paradox in the General Formula of Minimum Error Conical Projections.}

There is a rather curious mathematical paradox in the general formula for the Minimum Error Conical l'rojections which deserves notice for its mathematical interest, though it is not a question that need trouble the practical map-maker. It will have been noticed that in the solution of the general case of a map covering a zone from a lower limit a to an upper limit $\beta$, the Calculus of Viriations exacts that the radial scale error shall vanish at both limits, i.e. $a \cdot d \theta=\mathrm{I}$ both when $\theta$ a and $\theta \quad \beta$. If however we make $a=0$ we approach the case of map extending to centre and in the case of the Zenithal Projection, as amended by (Carke, we get for the radiul scale at centre, not the value $\mathrm{I}$, but the same as the perpendicular scalt:

$$
r \sin \theta \quad 1+\frac{\beta^{*}}{16} \cdots
$$

Again, in the General Conical Class the condition of Minimum Liror leads to two standard parallels and a mean spluare error $=\frac{n^{2}}{4+5}$; hut if we start with the assumption that the map extends to the centre, we are led w a projection with one standard paralkel only and a ment spunce error $=\frac{\beta^{2}}{\sqrt{765}}$. Now if in the former value for $\mu$ we athew a to alproach the value zero, then ox approaches the value $\frac{1}{2} \beta$ and $\frac{\delta^{2}}{4}+5$ apponchen the value $\frac{\beta^{\prime}}{\sqrt{720}}$ which is slighty larger. I'roferior Nicholson was kind anougle to 
give some attention to this paradox, and without going into a full investigation, the explanation seems to be that so long as we have a value for $\alpha$, however small, the projection will have the radial scale true at $a$ and two standard parallels; but when $\alpha$ is actually zero a discontinuity takes place and we have a projection with only one standard parallel. The paradox seems to be somewhat similar to that of the deflection of a circular elastic plate supported round the edge. If there is a hole in the centre however small we get a different result from that derived from the initial assumption of plate continuous to the centre. 


\section{CHAPTER III}

\section{THE CONICAL ORTHOMORPHIC PROJECTION WITH TWO STANDARL) PARALLELS (LAMIIERTS SECOND) FOR THE SPHEROII)}

We have hitherto considered the Earth to le a spluere of radius $k$, titken as unity in our formulac for brevity, but as it is usual, esperially in the projections employed for the rectangulat coordinates of triangulation, to take account of the excentricity, we will now investigate the expansions of the spheroidal formulae for the above. The expansions of the formulate for the case of one standard parallel are given by Jordan (1). $521-4$ ), l'iaretti (pp. 389-9I) and Oscar S. Adams, Special J'ublication No. +7 U. s. Coast and Geodetic Survey.

The formula for the spheroid in terms of our original notation is

$$
r=2 A\left(\tan 2^{1}(\theta)^{\prime \prime}\left(\begin{array}{c}
1+c \cos \theta \\
1-c \cos \theta
\end{array}\right)^{\prime 2},\right.
$$

where $e$ is the Eartl's excentricity. It will be now more convenient to put $K$ for the constant $2 A$ and write

$$
r=K\left(\tan \frac{1}{2} \theta\right)^{n}\left(\begin{array}{l}
\mathrm{I}+e^{\prime} \cos \theta \\
\mathrm{I}-e^{\prime} \cos \theta
\end{array}\right)^{n e} .
$$

Calling the radius of curvature to the meridian $\rho$, and the grand nomal or radius of curvature at right angles to the meridian 1 , we have

$$
\rho=a\left(\mathrm{I}-e^{2-2}\right)\left(\mathrm{I}-e^{2} \cos ^{2} \theta\right)^{32}, \text { and } v=a\left(1-e^{22} \cos ^{2} \theta\right)^{\frac{1}{2}},
$$

where $a$ is the semi-axis major of the spheroid. The scale along the meridian or what we have hitherto called the raclial scale, is given by $d r d m$, where $d_{m}$ is an element of the projected meridian. Noy

$$
d r d m \cdot d r \cdot d \theta \cdot d \theta \cdot d m=\frac{1}{p} d r \quad d r\left(\mathrm{I}-t^{2} \mathrm{cos} s^{\prime \prime} \theta\right)^{2} .
$$

I) ifferentiating logarithmically we have, putting $r^{\prime}$ for $d r d t, r^{\prime \prime}$ for $t^{\prime \prime} r d^{\prime \prime}$, eic,

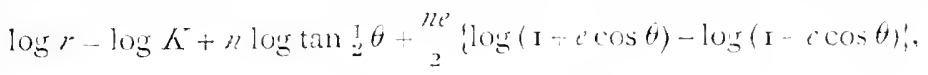

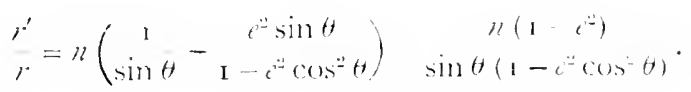

Therefore

$$
\text { d) } d m n \operatorname{nr}\left(\mathrm{I}-\cos ^{2} \theta\right)^{\frac{2}{2}} \text {. }
$$

and as the projection is orthomorphic the scale along the parallels has the same value. P'utting as before $\lambda$ for the co-hatitude of the mid parallel and 
$\delta$ for the angle from $\chi$ to standard parallels $\theta_{1}, \theta_{2}$, we have $\theta_{1}=\chi-\delta$, $\theta_{2}=\chi+\delta$. The condition that the scale at $\theta_{1}$ shall be the same as the scale at $\theta_{2}$ is

$$
n r_{1} \frac{\left(1-e^{2} \cos ^{2} \theta_{1}\right)^{\frac{1}{2}}}{a \sin \theta_{1}}=n r_{2} \frac{\left(1-e^{2} \cos ^{2} \theta_{2}\right)^{\frac{1}{2}}}{a \sin \theta_{2}},
$$

where $r_{1}, r_{2}$ are the radii at $\theta_{1}, \theta_{2}$. Writing out the full expressions for $r_{1}, r_{2}$ and cancelling common factors this leads to

$$
\begin{aligned}
& n=\frac{\log \sin \theta_{2}-\log \sin \theta_{1}-\log \left(\mathrm{I}-e^{2} \cos ^{2} \theta_{2}\right)^{\frac{1}{2}}+\log \left(\mathrm{x}-e^{2} \cos ^{2} \theta_{1}\right)^{\frac{1}{2}}}{e} . \\
& \log \tan \log _{2} \theta_{2}-\log \tan !_{2}^{1} \theta_{1}+\log \left(\frac{\mathrm{I}+e \cos \theta_{2}}{1-e \cos \theta_{2}}\right)^{2}-\log \left(\begin{array}{c}
\mathrm{I}+e \cos \theta_{1} \\
\mathrm{I}-e \cos \theta_{1}
\end{array}\right)^{2}
\end{aligned}
$$

The additional condition that the scale shall have the value unity along these parallels is

which leads to

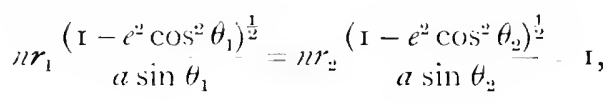

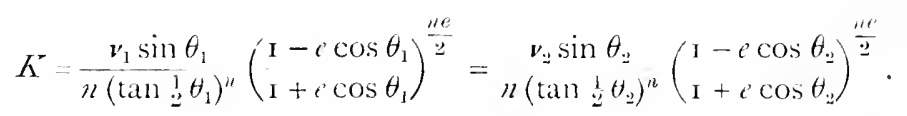

It will also be noted that the radii of the standard parallels have the simple values $r_{1}=r_{1} \sin \theta_{1} / n, r_{2}=r_{2} \sin \theta_{2} / n$. Such are the complete formulac for two standard parallels chosen beforehand. To calculate the values of $r$ by them with exactitude log tables of at least $S$ figures are required, so that in practice it is more convenicht and even more accurate to use expansions, involving the differences and values at the mid-parallel. These expansions can be made in terms of either the difference of latitude $\Delta \theta$ following Jordan or the difference of meridian distance $\Delta m$ following Pizzetti, the U.S. Survey and others. These writers consider the case of one standard parallel only, where $n=\cos x$,

$$
\Lambda=\frac{r_{x} \sin \chi}{n\left(\tan \frac{1}{2} \chi\right)^{\prime \prime}}\left(\begin{array}{c}
1-e \cos \chi \\
1+e \cos \chi
\end{array}\right)^{n !} .
$$

It maly bemarked that if we write

$$
\tan \frac{1}{2} \zeta-\tan 2_{2}^{1} \theta\left(\begin{array}{l}
r+e \cos \theta \\
1-e \cos \theta
\end{array}\right)^{-3}
$$

then $\zeta$ is very nearly the seocentric co-latitude

$$
\theta+\frac{1}{2} e^{2} \sin 2 \theta+\frac{1}{2} e^{4} \sin 2 \theta \cos ^{2} \theta+\ldots
$$

whereas by fxpanding the above expression we find

$$
\zeta=\theta+\frac{1}{2} r^{2} \sin 2 \theta+\frac{i}{2} e^{2} \sin 2 \theta \cos ^{2} \theta+\ldots
$$

as pointed out by Her\% (]) 2. f6). If tables of geocentric co-latitule are used therefore which take arcount of the term in $e^{4}$ the crror is very small, the maximum being only $0^{\prime \prime} \cdot 5$ for $\theta$ 30". 
By expanding the expressions involving $e$ we can write

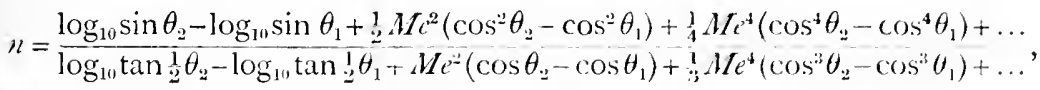

which is fairly easy to compute, $M /$ being the modulus of common logarithms.

But for our purpose we recpuire to expand the whole expression for $n$ in terms of $\delta^{2}$ and $e^{2}$ which can be done as follows:

$\log \sin \theta_{2}-\log \sin \theta_{1}=\int_{\theta_{1}}^{\theta_{2}} \cot \theta d \theta$

$$
2 \delta \cot \chi\left(1+\frac{\delta^{2}}{3 \sin ^{-2} \chi}+\frac{\delta^{4} 2+\cos ^{2-1} \chi}{\text { I } 5} \sin ^{4} \chi \ldots\right)
$$

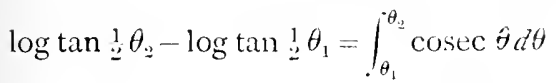

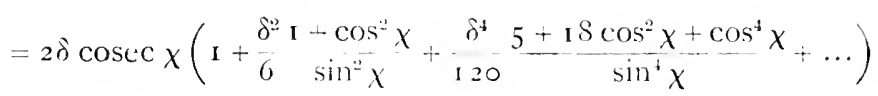

${ }_{2}^{1} e^{2}\left(\cos ^{2} \theta_{2}-\cos ^{2} \theta_{1}\right)=-e^{2} \int_{\theta_{1}}^{\theta_{2}} \sin \theta \cos \theta d \theta=-2 \dot{\partial} e^{2}\left(\sin \chi \cos \chi-\frac{2 \delta^{2}}{3} \sin \chi \cos \chi \ldots\right)$

$e^{2}\left(\cos \theta_{2}-\cos \theta_{1}\right)=-\left.e^{2}\right|_{\theta_{1}} ^{\theta_{2}} \sin \theta d \theta=-2 \delta e^{2}\left(\sin \chi-\frac{\delta^{2}}{6} \sin \chi \ldots\right)$.

We shall not carry the expansions beyond $\delta^{4}$ and $e^{2} \delta^{2}$, but if desired the further proceedings are obvious. I)ividing we find

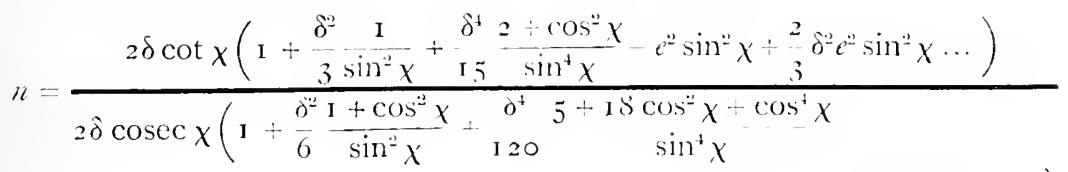

$$
\begin{aligned}
& \left.e^{22} \sin ^{2} x+\frac{1}{6} \partial^{2} e^{2 \prime} \sin ^{2} x \ldots\right)
\end{aligned}
$$

$\cos x\left\{1+\frac{\delta^{2}}{6}\left(1+t^{2} \sin ^{2} \chi\right)+\delta^{4} 60 \cdot \frac{16+7 \sin ^{2} x}{\sin ^{2} x} \ldots\right\}$.

We shall now continue the expansion of $r$, but it will be convenient to do this in terms of $\Delta m$, the meridian distance, insteral of $\Delta \theta$, the difference of co-latitude. We have found already $d r d m \quad n r\left(1 \quad e^{2} \cos ^{2} \theta\right)^{2} d x \sin \theta$, and differentiating further logarithmically, dropping the factor a fro tim., we have

$$
r^{\prime \prime}\left(\begin{array}{l}
r^{\prime} \\
r
\end{array}\right) \quad-n \cos \theta\left(1 \quad i^{2}\right),
$$

and multiplying by $d \theta d m$,

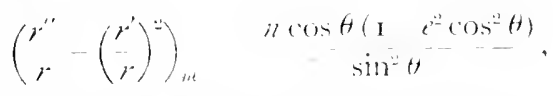

therefore $\quad\left(\frac{r^{\prime \prime}}{r}\right)_{m} \frac{n(n-\cos \theta)\left(1-e^{2} \cos \theta \theta\right)}{\sin ^{\circ} \theta}$. 
Continuing

$$
\begin{gathered}
\frac{r^{\prime \prime \prime}}{r}-\frac{r^{\prime \prime} r^{\prime}}{r^{2}}=n\left\{\frac{\mathrm{I}-e^{2} \cos ^{2} \theta}{\sin \theta}-\frac{\left(\mathrm{I}-e^{2}\right) 2 \cos \theta(n-\cos \theta)}{\sin ^{3} \theta}\right\}, \\
\left(\frac{r^{\prime \prime \prime}}{r}-\frac{r^{\prime \prime} r^{\prime}}{r^{2}}\right)_{m}=n\left\{\frac{\left(\mathrm{I}-e^{2} \cos ^{2} \theta\right)^{5}}{\sin \theta\left(\mathrm{I}-e^{2}\right)}-\frac{2 \cos \theta(n-\cos \theta)\left(\mathrm{I}-e^{2} \cos ^{2} \theta\right)^{\frac{3}{2}}}{\sin ^{3} \theta}\right\} . \\
\left(\frac{r^{\prime \prime} r^{\prime}}{r^{2}}\right)_{m}=\frac{n^{2}(n-\cos \theta)\left(\mathrm{I}-e^{2} \cos ^{2} \theta\right)^{3}}{\sin ^{3} \theta},
\end{gathered}
$$

But

therefore

$$
\left(\frac{r^{\prime \prime \prime}}{r}\right)_{m}=n\left\{\begin{array}{c}
\left(\mathrm{I}-e^{2} \cos ^{2} \theta\right)^{\frac{\pi}{2}} \\
\sin \theta\left(\mathrm{I}-e^{2}\right)
\end{array}+\frac{(n-\cos \theta)(n-2 \cos \theta)\left(\mathrm{I}-e^{2} \cos ^{2} \theta\right)^{\frac{3}{2}}}{\sin ^{3} \theta}\right\} .
$$

Again

$$
\begin{aligned}
& \frac{r^{\mathrm{iv} v}}{r}-\frac{r^{\prime \prime \prime} r^{\prime}}{r^{2}}=n\left\{\frac{5 e^{2}\left(\mathrm{I}-e^{23} \cos ^{2} \theta\right)^{32} \cos \theta \sin ^{2} \theta-\cos \theta\left(\mathrm{I}-e^{2} \cos ^{2} \theta\right)^{\frac{5}{2}}}{\sin ^{2} \theta\left(\mathrm{I}-e^{2}\right)}\right. \\
& +\frac{(n-2 \cos \theta)\left(\mathrm{I}-e^{2} \cos ^{2} \theta\right)^{3}}{\sin ^{2} \theta}+\frac{2(n-\cos \theta)\left(\mathrm{I}-e^{2} \cos ^{2} \theta\right)^{\frac{3}{2}}}{\sin ^{2} \theta} \\
& +\frac{3^{2} \cos \theta\left(1-e^{2} \cos ^{2} \theta\right)^{\frac{1}{2}}(n-\cos \theta)(n-2 \cos \theta)}{\sin ^{2} \theta} \\
& \left.-\frac{3 \cos \theta(n-\cos \theta)(n-2 \cos \theta)\left(\mathbf{I}-e^{2} \cos ^{2} \theta\right)^{\frac{3}{2}}}{\sin ^{4} \theta}\right\},
\end{aligned}
$$

and

$$
\begin{aligned}
& \left(\frac{r^{\mathrm{iv}}}{r}-\frac{r^{\prime \prime \prime} r^{\prime}}{r^{2}}\right)_{m}=n\left\{\begin{array}{c}
-\cos \theta\left(1-e^{2} \cos ^{2} \theta\right)^{4}+5 e^{2} \cos \theta \sin ^{2} \theta\left(1-e^{2} \cos ^{2} \theta\right)^{3} \\
\sin ^{2} \theta\left(1-e^{2}\right)^{2}
\end{array}\right. \\
& +\frac{(n-2 \cos \theta)\left(\mathrm{I}-e^{2} \cos ^{2} \theta\right)^{3}}{\sin ^{2} \theta\left(\mathrm{I}-e^{2}\right)}+\frac{2(n-\cos \theta)\left(\mathrm{I}-e^{2} \cos ^{2} \theta\right)^{3}}{\sin ^{2} \theta\left(\mathrm{I}-e^{2}\right)} \\
& +3^{e^{2}} \cos \theta\left(1-e^{2} \cos ^{2} \theta\right)^{2}(n-\cos \theta)(n-2 \cos \theta) \\
& \sin ^{2} \theta\left(\mathrm{I}-e^{-2}\right)
\end{aligned}
$$

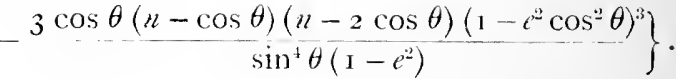

Now

$$
\left(\begin{array}{c}
r^{\prime \prime \prime} r^{\prime} \\
r^{2}
\end{array}\right)_{\prime \prime \prime}=u^{2}\left\{\begin{array}{c}
\left(1-e^{2} \cos ^{2} \theta\right)^{3} \\
\sin ^{2} \theta\left(1-e^{2}\right)
\end{array}+\frac{(n-\cos \theta)(n-2 \cos \theta)\left(1-e^{2 \prime} \cos ^{2} \theta\right)^{2}}{\sin ^{4} \theta}\right\},
$$

therefore

$$
\begin{aligned}
& \left(\begin{array}{c}
r^{\mathrm{iv}} \\
r
\end{array}\right)_{m}=u\left\{\frac{-\cos \theta\left(1-e^{2} \cos ^{2} \theta j^{4}+5 i^{2-2} \cos \theta \sin ^{2} \theta\left(1-e^{22} \cos ^{2} \theta\right)^{*}\right.}{\sin ^{2} \theta\left(1-i^{2}\right)^{2}}\right. \\
& +(n-\cos \theta)\left(1-e^{2} \cos ^{2} \theta\right)^{2} \\
& \sin ^{2} \theta\left(1-i^{2}\right) \\
& +3 c^{2} \cos \theta(n \cos \theta)(n-2 \cos \theta)\left(1-e^{2} \cos ^{2} \theta\right): \\
& \sin ^{2} \theta\left(1-i^{2 *}\right) \\
& -\frac{3 \cos \theta(n-\cos \theta)(n-2 \cos \theta)\left(1-e^{2} \cos ^{2} \theta\right):}{\sin ^{2} \theta\left(1 \quad i^{2}\right)} \\
& n(n-\cos \theta)(n-2 \cos \theta)\left(1-i^{2} \cos ^{2} \theta\right)^{2} \\
& \sin ^{+} \theta
\end{aligned}
$$




$$
\begin{aligned}
& =n\left\{\frac{-\cos \theta\left(\mathrm{I}-e^{2} \cos ^{2} \theta\right)^{3}\left(1-e^{2}-4 e^{2} \sin ^{2} \theta\right)}{\sin ^{2} \theta\left(\mathrm{I}-e^{2}\right)^{2}}\right. \\
& +\frac{+(n-\cos \theta)\left(\mathrm{I}-e^{2} \cos ^{2} \theta\right)^{3}}{\sin ^{2} \theta\left(\mathrm{I}-e^{2}\right)} \\
& -\frac{3 \cos \theta(n-\cos \theta)(n-2 \cos \theta)\left(1-t^{2} \cos ^{2} \theta\right)^{2}}{\sin ^{2} \theta}
\end{aligned}
$$

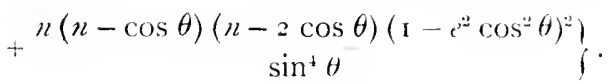

Now if we are expanding only as far as $m^{4}$ we can put $n-\cos \chi$ in the expressions for $\left(r^{\prime \prime \prime} / r\right)_{m}$ and $\left(r^{i v} / r\right)_{m}$, so that only the first terms within the brackets will be retained. Since also

we have

$$
\rho=a\left(\mathbf{I}-e^{2}\right)\left(\mathrm{I}-i^{2} \cos ^{2} \theta\right)^{2}, \quad v-a\left(\mathrm{I}-c^{2} \cos ^{2} \theta\right)^{\frac{1}{2}},
$$

$$
\rho v=a^{2}\left(\mathrm{I}-e^{2}\right) /\left(\mathrm{I}-e^{2} \cos ^{2} \theta\right)^{2}, \quad \rho^{2} \cdot a^{3}\left(\mathrm{I}-e^{2}\right)\left(\mathrm{I}-e^{2} \cos ^{2} \theta\right)^{2} .
$$

Thus, replacing $\theta$ by the value $\chi$ for mid-parallel, we have

$$
\begin{aligned}
& r=K\left(\tan \frac{1}{2} \chi\right)^{\prime \prime}\left(\frac{\mathrm{I}+e \cos \chi}{\mathrm{I}-e \cos \chi}\right)^{\frac{n}{2}}\left\{\begin{array}{c}
\mathrm{I}+n\left(\mathrm{I}-e^{2 \prime} \cos ^{2} \chi\right)^{\frac{1}{2}} \\
a \sin \chi
\end{array}\right. \\
& +\frac{n(n-\cos \chi)\left(\mathrm{I}-e^{2} \cos ^{2} \chi\right)}{a^{2} \sin ^{2} \chi} \cdot \frac{m^{2}}{2}+\frac{n\left(1-e^{2} \cos ^{2} \chi\right)^{2}}{a^{2} \sin \chi\left(1-i^{2}\right)} \cdot \frac{m^{\prime \prime}}{6}
\end{aligned}
$$

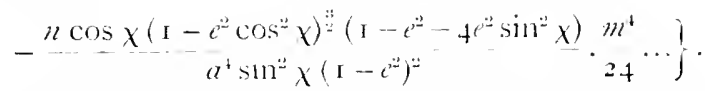

We shall subsequently expand $K$ in terms of $\chi$ and $o$ but for the moment we put

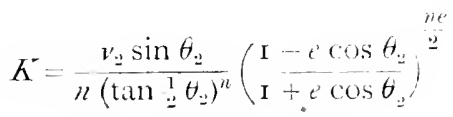

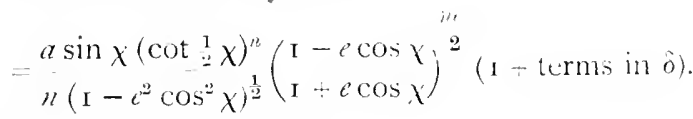

We then have radius at micl-parallel

$$
r_{x} \frac{a \sin \chi}{n\left(1-e^{22} \cos ^{2} \lambda^{2}\right)^{\frac{1}{2}}}(1+\text { terms in } \delta),
$$

and

$$
\begin{aligned}
& r-r_{x}=\left\{m+\frac{(r-\cos x)\left(1-i^{2} \cos ^{2} x\right)^{\frac{1}{2}} \cdot m^{2}}{a \sin x}+\begin{array}{c}
\left(1-i^{2} \cos ^{2} x\right)^{\prime} \\
a^{2}\left(1-i^{2}\right)
\end{array} m^{2}\right.
\end{aligned}
$$

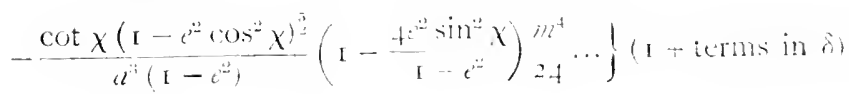

$$
\begin{aligned}
& \left\{m+\frac{(n-\cos \gamma)}{1 \cdot \sin \chi} \cdot m^{2}+\frac{1}{\rho} \cdot \frac{m}{6}\right.
\end{aligned}
$$

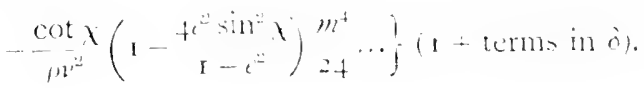


For $n=\cos \chi$ this reduces to the expression found by Pizzetti for one standard parallel, when terms in $\delta$ vanish. Substituting however the approximate value of

$$
n=\cos \chi\left\{\mathrm{I}+\frac{\delta^{2}}{6}\left(\mathrm{I}+4 e^{2} \sin ^{2} \chi\right) \ldots\right\}
$$

we find the second term gives a term in $m^{2} \delta^{2}$ and

$$
\begin{aligned}
r-r_{\chi}=\left\{m+\frac{\mathrm{I}}{\rho \nu} \cdot \frac{m^{3}}{6}+\frac{\cot \chi}{v}(\mathrm{I}\right. & \left.+4 e^{2} \sin ^{2} \chi\right)^{\frac{m^{2} \delta^{2}}{\mathrm{I} 2}} \\
& \left.\quad-\frac{\cot \chi}{\rho \nu^{2}}\left(\mathrm{I}-4 e^{2} \sin ^{2} \chi\right) \frac{m^{4}}{24} \ldots\right\}(\mathrm{I}+\text { terms in } \delta) .
\end{aligned}
$$

We must now expand the expression for $K$ in order to find the terms in $\delta$. We have

$$
K=\frac{a \sin \theta_{2}\left(\cot \frac{1}{2} \theta\right)^{n}}{n\left(1-e^{2} \cos ^{2} \theta_{2}\right)^{\frac{1}{2}}}\left(\begin{array}{c}
\mathrm{I}-e \cos \theta_{2} \\
\mathrm{I}+e \cos \theta_{2}
\end{array}\right)^{\frac{n e}{2}}
$$

Now $\quad \sin \theta_{2}=\sin \chi\left(I+\cot \chi \cdot \delta-\frac{\delta^{2}}{2}+\frac{\delta^{2}}{6} \cot \chi \ldots\right)$,

also

$$
\begin{aligned}
& \left(\cot 1 \theta_{2}\right)^{n}=(\cot 2 \chi)^{\prime \prime}\left(1-\frac{n}{\sin \chi} \delta+\frac{n^{2}+n \cos \chi}{\sin ^{2} \chi} \cdot \frac{\delta^{\prime \prime}}{2}\right. \\
& \left.-\frac{n^{3}+3 n^{2} \cos \chi+n+n \cos ^{2} \chi}{\sin ^{3} \chi} \cdot \frac{\delta^{\prime \prime}}{6} \cdots\right)
\end{aligned}
$$

and putting in the proper value of $n$ this becomes

$$
\begin{aligned}
& \left(\cot \stackrel{2}{2}_{2}^{2}\right)^{n}=\left(\cot \underline{2}_{2}^{\prime} \chi\right)^{\prime \prime}\left(\mathbf{I}-\cot \chi \cdot \delta+\cot ^{2} \chi \cdot \delta^{\prime 2}\right.
\end{aligned}
$$

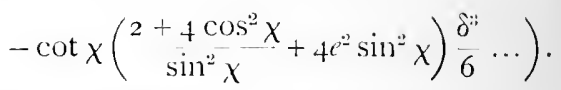

Multiplying this by the expansion for $\sin \theta_{i}$ we find

$$
\sin \theta_{2}\left(\cot \stackrel{1}{2} \theta_{2}\right)^{n}=\sin \chi\left(\cot \frac{1}{2} \chi\right)^{n}\left(1-\frac{\delta^{2}}{2}-\frac{2}{3} e^{2} \sin \chi \cos . \delta^{: 3} \ldots\right) \text {. }
$$

Next take $\left(1-e^{-2} \cos ^{2} \theta_{i:}\right)^{-\frac{1}{2}}=f$ say, we have

$$
\log f=-\stackrel{1}{:} \log \left(1-e^{2} \cos ^{2} \theta_{22}\right), \quad f^{\prime} / f=-\frac{e^{2} \sin \theta \cos \theta}{1-e^{2} \cos ^{2} \theta}=-e^{2} \sin \theta \cos \theta,
$$

neglecting terms above $e^{2 \prime} ; f^{\prime \prime}-\left(\begin{array}{l}f^{\prime} \\ f\end{array}\right)^{2}=-e^{2}\left(\cos ^{2} \theta-\sin ^{2} \theta\right)=\frac{f^{\prime \prime}}{f}$ to same order, and $f_{f}^{\prime \prime \prime}=4 e^{2 \prime} \sin \theta \cos \theta$ also to same order, therefore

$$
\begin{aligned}
& \left(1-e^{2} \cos ^{2} \theta_{2}\right)^{-1}-\left(1-i^{2} \cos ^{2} \chi\right)^{-\frac{1}{2}}\left(\mathrm{I}-e^{2} \sin \chi \cos \chi \cdot \delta\right. \\
& \left.-e^{2}\left(\cos ^{2} \chi-\sin ^{2} \chi\right) \frac{\delta^{2}}{2}+t^{2} \sin \chi \cos \chi \cdot \frac{2 \delta^{\prime \prime}}{6} \cdots\right) \text {. }
\end{aligned}
$$




\section{LAMBERT'S SECOND PROJECTION}

In the same way we shall find

$$
\begin{aligned}
& \left(\frac{\mathrm{I}-e \cos \theta_{2}}{\mathrm{I}+e \cos \theta_{2}}\right)^{\frac{n e}{2}}=\left(\begin{array}{l}
\mathrm{I}-e \cos \chi \\
\mathrm{I}+e \cos \chi
\end{array}\right)^{\frac{n e}{2}}\left(\mathrm{I}+\mu c^{2} \sin \chi^{\delta}+\mu \epsilon^{2} \cos \chi \cdot \delta_{2}^{\delta^{2}}\right. \\
& \left.-n e^{2} \sin \chi \cdot \frac{\delta^{*}}{6} \ldots\right)
\end{aligned}
$$

$=\left(\frac{1-e \cos \chi}{1+e^{2} \cos \chi}\right)^{\frac{n e^{\prime}}{2-}}\left(1+e^{2} \sin \chi \cos \chi \cdot \delta+e^{2 \prime} \cos ^{2} \chi \cdot \frac{\delta^{2}}{2}+\right.$ terms in $\delta^{4}$ upwards $)$.

Nultiplying out we get

$\left(\frac{\mathrm{I}-e \cos \theta_{2}}{\mathrm{I}+c \cos \theta_{22}}\right)^{\frac{n}{2}}\left(\mathrm{I}-e^{2} \cos ^{2} \theta_{2 .}\right)^{-\frac{1}{2}}$

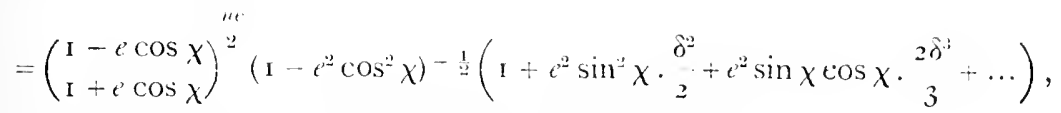

and multiplying this by the expression above we get

$$
\begin{array}{r}
\frac{a \sin x(\cot 2 x)^{n}}{n\left(1-e^{2} \cos ^{2} x\right)^{\frac{1}{2}}}\left(\frac{1-e \cos x}{1-e \cos x}\right)^{\frac{m}{2}}\left(1-\frac{1}{2} \delta^{2}\left(1-e^{2} \sin ^{2} \chi\right)\right. \\
\text { + terms in } \delta^{4} \text { upwards!. }
\end{array}
$$

We can test the accuracy of these expansions by seeing if the scale works out correct on the standard parallels; we have

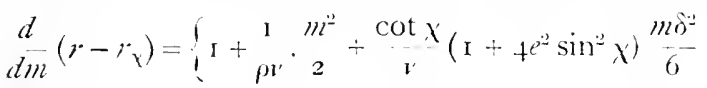

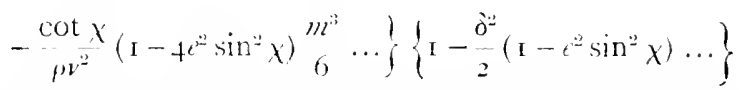

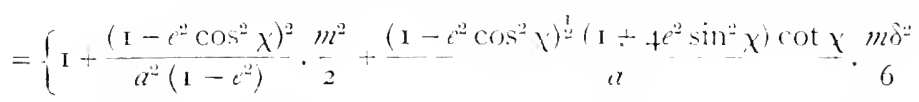

$$
\begin{aligned}
& \left.-\frac{\left(1-e^{2} \cos ^{2} x\right)^{\frac{2}{2}}\left(1-4 e^{2} \sin ^{2} x\right) \cot x}{a^{i 3}\left(1-e^{2}\right)} \cdot \frac{m^{i}}{6} \ldots\right)\left(1-\frac{\delta^{2}}{2}\left(1-e^{2} \sin ^{2} x\right) \cdots\right\}^{\prime} .
\end{aligned}
$$

Now

$$
\begin{aligned}
& m=\rho o\left(1-\frac{3 \rho}{2 y} \cdot e^{2} \sin \times \cos x \cdot \hat{o} \ldots\right) \\
& =\frac{a\left(1-e^{2}\right) j}{\left(1-t^{2} \cos ^{2} \chi\right)^{2}}\left(1-t^{2} \sin x \cos x \cdot \delta \ldots\right):
\end{aligned}
$$

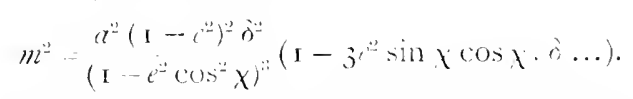

substituting these and neglecting powers of ialowe $e^{-2}$ we get for the scale

$$
\begin{aligned}
& \left\{\mathbf{r}+\left(1-e^{2} \sin ^{2} \chi\right)_{2}^{\delta^{2}}-t^{2} \sin x \cos \chi \cdot 0^{*}(:-1-1) \ldots\right\} \\
& \left.\sum_{1}^{1-j_{2}}\left(1+\sin ^{2} \gamma\right) \cdots\right)
\end{aligned}
$$

which proves our formulac. 
If we write $m_{0}$ for the mean of the meridional distances from mid-parallel to standard parallels we have $m_{0}=\rho \delta+$ terms in $e^{2} \delta^{3}$ upwards, so that $\delta^{2}=\frac{m_{0}{ }^{2}}{\rho^{2}}$ to same order and

$$
\delta^{2}\left(1-e^{2} \sin ^{2} \chi\right)=\frac{m_{0}^{2}\left(1-e^{2} \sin \chi\right)\left(1-e^{2} \cos ^{2} \chi\right)^{3}}{a^{2}\left(1-e^{2}\right)^{2}}=\frac{m_{0}^{2}}{\rho \nu}
$$

up to terms in $e^{2}$ inclusive, so that we can write

$$
\begin{aligned}
\left(r-r_{\chi}\right)=\left\{m+\frac{m^{3}}{6 \rho \nu}-\frac{m^{4}}{24 \rho \nu^{2}} \cot \chi\left(\mathrm{I}-4 e^{2} \sin ^{2} \chi\right)+\frac{m^{2} m_{0}{ }^{2}}{\mathrm{I} 2 \rho \nu^{2}}\left(\mathrm{I}+4 e^{2} \sin ^{2} \chi\right) \ldots\right\} & \\
& \times\left\{\mathrm{I}-m_{0}{ }^{2} \ldots\right\} .
\end{aligned}
$$

The value of $r_{\chi}$ is

$$
\begin{gathered}
\quad a \sin \chi \\
n\left(\mathrm{I}-e^{2} \cos ^{2} \chi\right)^{\frac{1}{2}}\left\{\mathrm{I}-\frac{\delta^{2}}{2}\left(\mathrm{I}-e^{2} \sin ^{2} \chi\right) \ldots\right\} \\
=v \tan \chi\left\{\mathrm{I}-\frac{2 \delta^{2}}{3}\left(\mathrm{I}+\frac{1}{4} e^{2} \sin ^{2} \chi\right)\right\} .
\end{gathered}
$$

The French in using these expansions for their new maps employ up to the term $m^{3} / 6 \rho v$ only, and for this purpose it is sufficient to take for $n$ the value $\cos \chi$ as for one standard parallel, the correction to make two parallels standard being effected by the multiplier $\left(\mathbf{r}-m_{0}^{2} / 2 \rho v^{\prime}\right)$. For $r_{0}$ they use the value $r \tan \chi\left(1-m_{0}^{2} / 2 \rho v\right)$. By some oversight they employ $\nu^{2}$ instead of $\rho \nu$ in their formulae. These small errors by a fortunate concurrence of opposing effects have an almost imperceptible result on their tables of coordinates.

The writer has expanded the formulae still further as for the sphere, that is, neglecting the terms depending on $e$. For $r-r_{0}$ there should be added $+\frac{m^{5}}{120 K^{4}} \frac{5-\cos x}{\sin ^{2} x}-\frac{m^{\prime \prime} \delta^{2}}{36 K^{4}} \frac{\cos ^{2} \chi}{\sin ^{2} \chi}$, and the reducing factor becomes

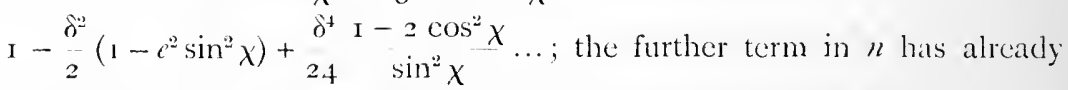
been given. If we take $\chi 90^{\circ}$ and put $\sin \chi=\mathrm{r}, \cos \chi-0$ we obtain the formulae for the Nercator I'rojection for the spheroid with two standard parallels in latitudes $\pm \delta$.

\section{Minimum Error formulae for the Spheroid.}

In the case of the ninimum error formulae for the spheroid we do not select the two standard parallels beforehand, but we make the scale values equal on the npper and lower parallels for one condition determining $n$, and for the other determining $K$ we make the total elepth of the map true. Now we have already in the abowe insestigation cletermined the formula for $"$, becaltse we have only to consicler $\delta$ as being no longer the angle: 
from mid-parallel to standard parallels but as the angle from middle to extreme parallels. To find $K$ we have

$$
r_{\beta}-r_{\alpha}=K\left\{\left(\tan \frac{1}{2} \beta\right)^{\prime \prime}\left(\frac{1+e \cos \beta}{1-e \cos \beta}\right)^{\frac{n}{2}}-\left(\tan \frac{1}{2} \alpha\right)^{\prime \prime}\left(\frac{1+e \cos \alpha}{1-e^{\prime} \cos \alpha}\right)^{2}\right\}=\int_{\alpha}^{\beta} \rho d \theta \text {. }
$$

We have already inclicated the method for expanding these expressions, which involve long and complicated differentiations and it will suffice here to give the result,

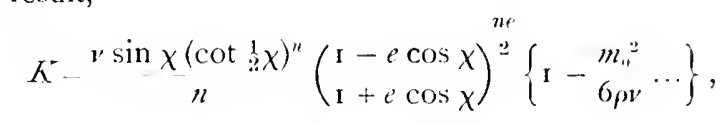

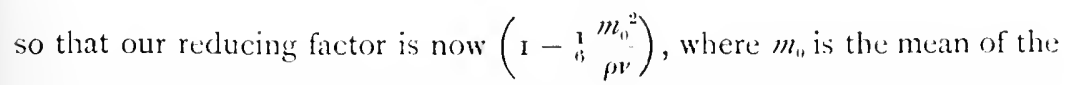
meridional distances of upper and lower parallels from the mid-parallel. The corresponding value of $r_{\gamma}$ is

$\frac{a \sin \chi}{n\left(1-t^{22} \cos ^{2} \chi\right)^{\frac{1}{2}}}\left\{1-\frac{\delta^{2}}{6}\left(1-c^{2-} \sin ^{2} \chi\right) \cdots\right\}=v \tan \chi\left\{1-\frac{\delta^{2}}{3}\left(1+3 \sin ^{2} \chi\right) \cdots\right\}$, where $\delta$ is now the angle from middle to extreme parallels.

It is obvious that by writing

$$
r-r_{\lambda}=m-\frac{m^{3}}{6 \rho v} \text { and } K-\ldots\left\{1+\frac{m_{0 i}^{*}}{6 \rho v}\right\},
$$

we shall obtain a projection which is (approximately) Equal Area such as would result from expanding Albers' minimum error formulae for the spheroid. 


\section{CHAPTER IV}

\section{THE POLYCONIC PROJECTIONS}

The Simple Polyconic Projection, which the French cartographers call the "projection polyconique ordinaire des Americains" because it seems to have been first introduced by the U.S. Coast and Geodetic Survey, has the following characteristics. A standard meridian of longitude is chosen which is usually the centre line of the map. This is developed in its true length on the map and each parallel of latitude is developed on its own tangent cone opened out on the plane of the map. Thus the standard meridian is true to scale on the map, and all the parallels cut it at right angles and preserve their true scale; but because the radii of the projected parallels vary as the cotangent of the latitude from point to point, the developed meridians on each side of the standard are no longer straight but curved lines concave to the central meridian and their lengths are too great in proportion as their difference of longitude from the central meridian increases. When this projection is used, however, for only a small distance on each side of the central meridian its errors are correspondingly small, and it has the advantage that concise Tables can be calculated once for all for plotting the coordinates of the graticule of any map. Extensive Tables of this kind have been published by the U.S. Coast and Geodetic Survey, for each minute of latitude from the Equator to the Pole, and for intervals of longitude up to $30^{\circ}$ from the central meridian, in the Report of that Survey for $188_{4}$. Full particulars of the theory as applied to the spheroid will be found in Special P'ublication No. 57 of that Survey. 'These 'l'ables were not used by the Committee for the International Map, as different values of the Earth's spheroidal elements were thought desirable. It seems to the writur very desirable that some international agrement should be come to in matters of this kind, as many valuable Tables have been calculated in different countries using different values of these elements, thus seriously vitiating their usefulness for purposes of comparison. Is the actual geoid differs considerably in places from any of the spheroids put forward from time to time, lut as these spheroids (liffer among themselves by quantities so small as hardly to concern the map-maker, the best course would appear to be to decide on which of them the most work has atready been done in the way of calculating 'Tables and to adopt that spheroid for all international purposes.

The efuivalence of the simple l'olyconic Projection with the cassini will be readily secen hy comparing the expressions for the expansions of the comdinates. Those for the (assini are given by fordan (1) fs3). The

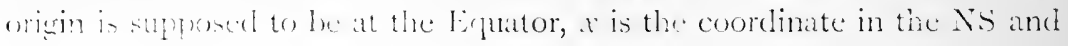


$y$ the coordinate in the $\mathrm{E}-\mathrm{W}$ direction positive $\mathrm{N}$ and $\mathrm{E}, \phi$ is the latitude and $\lambda$ the longitude (reckoned from the central meridian in c.m.) of a point on the map, $M$ is the meridian distance of the point from the Equator (called $B$ by Jordan), $v$ the grand normal at latitude $\phi$. Then we have

Cassini $\quad x=L I+\frac{1}{2} v \lambda^{2} \sin \phi \cos \phi+1 \lambda^{4} \sin \phi \cos \phi\left(5-6 \sin ^{2} \phi\right)+\ldots$,

Polyconic $x=. K+]_{2}^{1} v^{2} \sin \phi \cos \phi-2_{2}^{1} v^{4} \lambda^{4} \sin ^{3} \phi \cos \phi \ldots$,

I)ifference $: v^{2} \lambda^{4} \sin \phi \cos ^{*} \phi \ldots$.

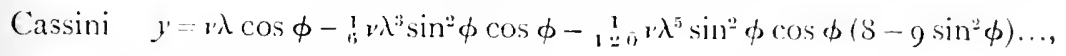

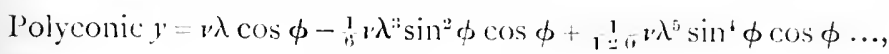

I)ifference ${ }_{1}^{1}: x^{5} \sin ^{2} \phi \cos ; \phi \ldots$

In the International Map of the World on the scale of I million the device was adopted of reducing the scale along the contral meridian by half the amount of the error at the extreme meridians, that is, by ${ }_{4}^{1} \lambda^{2} \cos ^{2} \phi_{m}$, where $\phi_{m}$ is the mean latitude of a sheet. This appears to have been regurded as a rather empirical corrention by its proposers, but the investigations we have made prove that it was really batsed on a losical criterion, that of making the maxinum error as small as possible. In the writer's opjinion, howeser, it would have been better to take as the reducing factor ${ }_{i}^{1} \lambda=\cos ^{2} \phi_{m}$, following Airy s criterion of making the average error a minimum and the total area true. But as a matter of fact the correction is on these sheets so small as to be within the errors caused by hygrometric changes in the paper. It may le noted here that in these Instructions and in Germain, $x$ is used for the E-W and $\mathrm{I}^{\prime}$ for the $\mathrm{N}-\mathrm{S}$ coordinates. The writer prefers to use these symbols the other way about, for if $u$ is the bearing of a line s nearly alwas reckoned from the North in survey work, we have the common forms $x-s \cos \alpha, y=s \sin \alpha$.

\section{Equal Area and (approx.) Orthomorphic Polyconic Projections.}

J. I. Craig has given on p. 6r of his Theory of Map Projertiens the formulat for a Polyconic Projertion which is Eipual Area. Insteal of the inclination of the projectel meridians being $\lambda \sin \phi$ as in the simple l'olyconic, this quantity becomes a series of which the first two terms are $\lambda \sin \phi\left(1-\lambda^{2} \cos ^{2} \phi \ldots\right)$.

By changing the sign of the second term and taking this puantity as

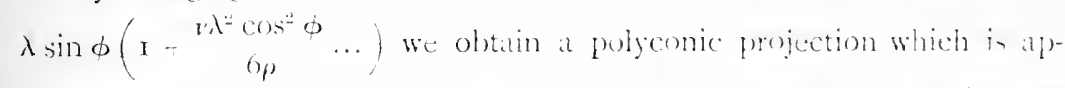
proximately orthomorphic. It will be foumed to asrec in fiet with the expansion of the lranstere Mercator or Ciasus ( onfomal Projection to the first term of the scale crrors.

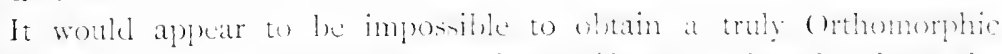
Polyconic l'rojection withut sacriticing unifomng of solk aloms the 
central meridian. Such a projection was invented by Lagrange and is usually known by his name. That it really belongs to the Polyconic Class is pointed out by Tissot and Herz, and also by Oscar S. Adams in Special Publication No. 57 of the U.S. Coast and Geodetic Survey.

\section{Lagrange's Projection for the Spheroid.}

The full investigation of Lagrange's P'rojection for the spheroid will be found in Fiorini (pp. 533 et seq.) and U.S. I'ublication No. 57, and it is not necessary to repeat it here. The principal meridian coordinate $x$ can be written in the form $x=\frac{A\left(k c^{n}-t^{n}\right) k}{k c^{n}+2+t^{n} / k}$, where $A, k$ and $n$ are constants to be determined and $c^{n}=\mathrm{I} / t^{n}$ is written for $\left(\cot \frac{1}{2} u\right)^{u}\left(\frac{\mathrm{I}-e \cos u}{\mathrm{I}+e \cos u}\right)^{2}, u$ being the co-latitude of a point on the meridian. The scale value $d x d m$ will be given by $2 n A / v \sin u\left(k c^{\prime \prime}+2+t^{\prime \prime} / k\right)$, and Lagrange chose the constants $k$ and $n$ so that the first and second differential coefficients of this quantity should vanish at the point of co-latitude $u_{0}$ chosen as the centre of the map. The first condition leads to $k c_{v}{ }^{n}=\frac{n+\cos u_{u}}{n-\cos u_{0}}$. From the second condition

Lagrange derives $n=\left(1+\sin ^{2} u_{0}\right)^{\frac{1}{2}}$, but in doing so he neglects the excentricity. Captain G. T. McCaw and the writer almost simultaneously found the correct value for $n$ should be $\left.n=\}_{1}+\sin ^{2} u_{10}+2 e^{2} \sin ^{4} u_{0}\left(1-e^{2}\right)\right\}^{\frac{1}{2}}$. The constant $A$ is usually chosen so that the scale shall be true at co-latitude $u_{v}$, but our investigations shew that a better result would be achieved by taking $A$ so that the scale error along the central meridian should be one-third that of opposite sign along the extreme meridians.

For a map of small extent in longitude Lagrange's formulae, when expanded, agree to the first term of scale errors with those of the Transverse Mercator, so there is no advantage in using it for maps of moderate size. The variation of scale along the central meridian is very slow. The writer finds

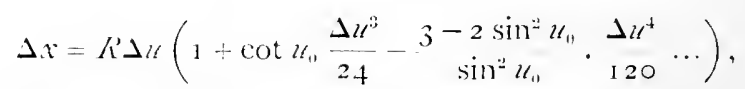

so that if the centre of the map) is on the liquator even the third differential coefficient of the scalc value vanishes.

This projection has some interesting geometrical properties, as the projected meridians and parallels are true circles even on the spheroid. It has this advantage over the Nercator that a map of the globe can be constructed on it without the srale error becoming infinite (which applies: also to all the P'olyeonic P'rojections) but the exaggeration from $80^{\circ}$ latitude to the Pole is conormous (compare l'rojections Nos. $v$ and 111 in ficrmain, l'lates VI and VII). 


\section{CHAPTER V}

\section{THE FINITE ERRORS OF PROJECTIONS}

As we have already remarked in (hat). II, the differential scale crrors at a point of a projection are not perhaps of much vatue to the users of maps. Cenerally speaking, the projection shoukl be so chosen that the greatest error in distance and bearing between two points at a finite distance apart taken from the map, shall be smaller than the accielental errors due to stretcining of the paper, ete., and imaller than the desree of accuracy required in the purpose for which the metsurement is recpuired. But when the projection is reguired for the purpose of fixing points with great accuracy by rectangular coordinates as for a caclastmal curvey, or esen for artillery work, it may be necessary to know how the distance and bearing derived from such rectangular coordinates differ from their trut values on the surface of the sphere or spheroicl.

For the Ciauss Conformal or 'Transterse Mercator l'rojection, as used in the surveys of Hanower and ligy pt, such formulac have becen worked out for the spheroid to a high denge of accuracy by (ieneral (Oscar Sehreiber in his Theorie der I'rojektionsmethede der Hannererschen Lambstermessung (Hannover, Hahn'sche 11 of huchbandlung 1860 ). They are also given as far as the fourth powers of coordinates by Jordan (Pp. 507-12). These formulae apply to the case of scale tme along the merician (or what we hale called one standard parallel in our inventigation of the (Conical Projections), but in the case of orthomorphism they can be applied to the similar projer tion with two standard parallels or submeridians without any change in computing the angular corrections, and for the rlstanes correction it will only be necensury, after computing the eorrection by these formulate, waply. with opporite sign the seakle reduction factor.

The same formulac cur lx used, with simple interchange of the a coordinates for the 1 , at any rate to the first term of the errors--which ought to be neat enomgin for all practical purposes to the lirect lereator. the

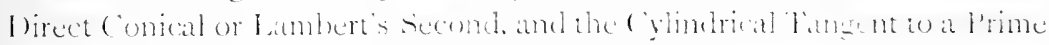

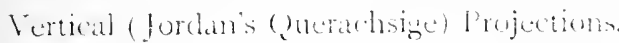

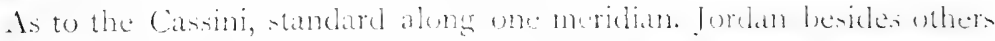

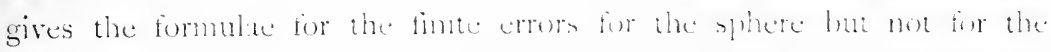

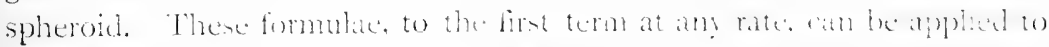

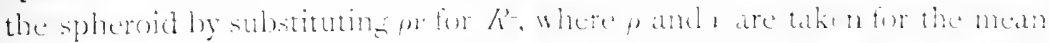

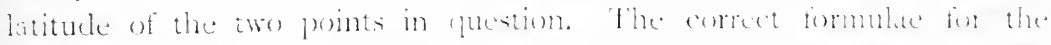

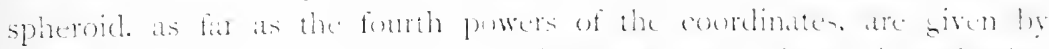

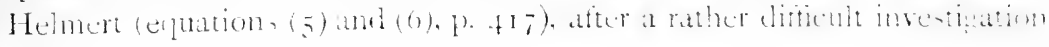


and in a rather difficult form. 'The writer succeeded in checking Helmert's result in two ways, first by a transformation from the coordinates of the

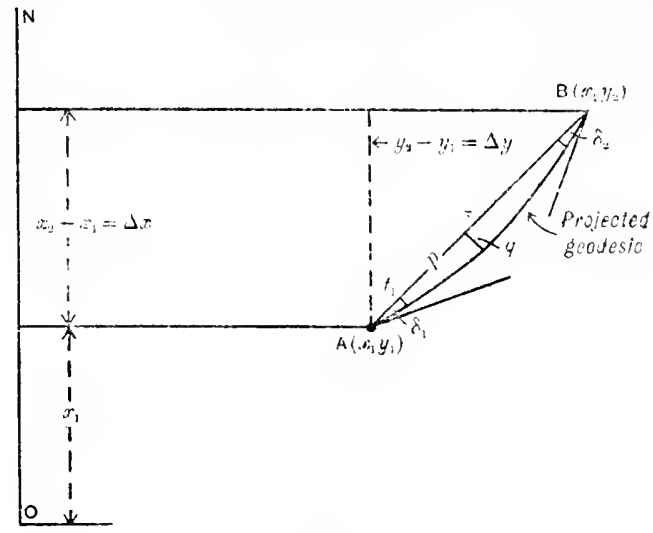

Fig. 2.

Gauss Conformal, and secondly by pursuing the methot of Causs (see Schreiber, 1) 39) of forming the differential equation of the curve assumed by the projected geodesic between the two points, ly the Calculus of Variations. This differential equation is more complex than that in the Conformal system, but a solution can be found in series. It can be written thus:

$$
-\frac{d^{2} q}{a^{2}}=\frac{y}{r_{1}^{2}}\left(1-\frac{+\left(x-x_{i}\right) \eta^{\prime \prime} t}{r^{2}}\right)\left(\cos ^{3} t_{1}+2 \sin ^{2} t_{1} \cos t_{1}\right)-\frac{2 \eta^{2} t y^{2} \sin t_{1} \cos ^{2} t_{1}}{r^{3}}
$$

the symbols having the same meaning as in Jorklan, 1). $5 \mathrm{Ir}$, where an abridgement of Schreiber's method is given. 'This equation when solved will give the angles between the chord joining the two points on the projection and the tangents to the projected geodesic: but to these mnst in addition be applied the change in the bearing of thic chord duc to the

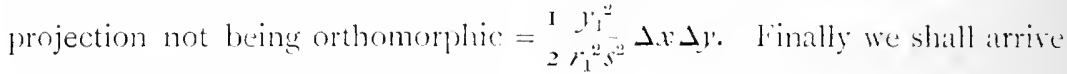
at the following ef puations, where

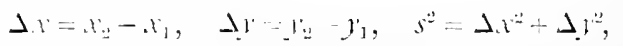

$S^{-}$sepuare of geotesic distance between the two points on the spberoid,

$r_{i}{ }^{\prime 2} \rho v^{\prime}$ at the point $x_{1} y_{1}$,

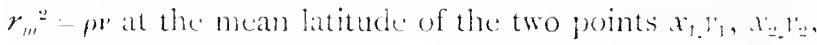

$\phi=$ that meith latitude.

$t \operatorname{tin} \phi, \quad \eta^{2}-i^{2 \prime} \cos ^{20} \phi\left(1-i^{2}\right)$,

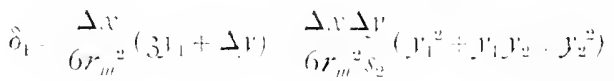

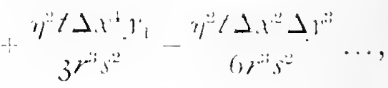




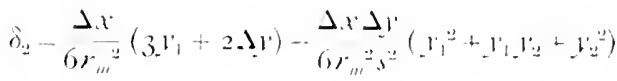

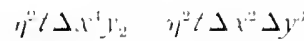

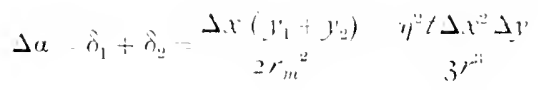

$$
\begin{aligned}
& \left.3^{3}\right)^{3} \quad 6, s^{3}=
\end{aligned}
$$

$$
\begin{aligned}
& S^{2} \cos ^{2} t_{1} \quad \begin{array}{l}
x^{2} \\
3 r_{m}^{2} \\
3 x^{2}
\end{array}
\end{aligned}
$$

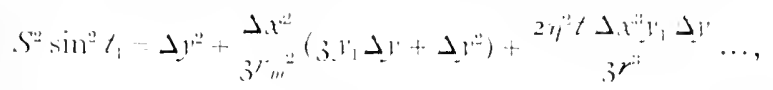

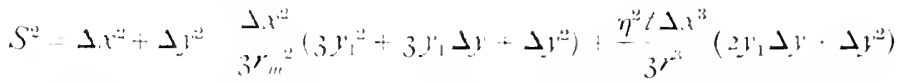

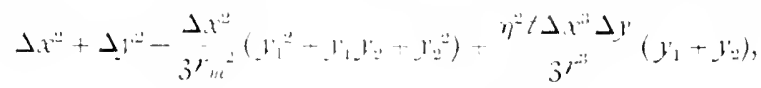

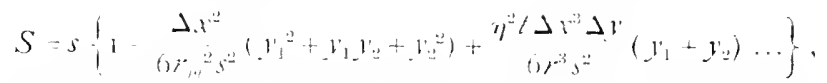

which agree with fletmert s formulat remembering that he reckons $x$ and $y$ as positive $S$ and $\mathbb{W}$.

The above expressions, as well as thone of Helnewt, fordan and Schreiber. refer to the sendesic lines on the spheroid and their lirections. Before using them the appropriate corrections should be mate to the observed directions, or we may add these corrections to the above expressions,

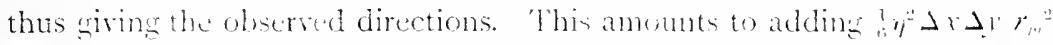

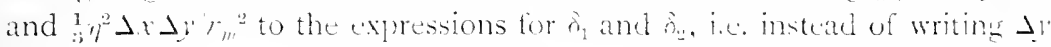
in the first tem of each we write $\left.-1 y^{2}(1+\eta)^{2}\right)$. As these corrections ate so small as to be generally neglected we mat well reject the higher terms in $y^{2}$, and use only the terme involving the second puwers of the eordinates, whth the proper valie of $r$.

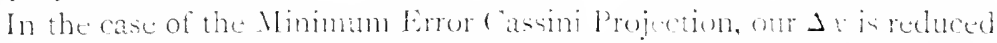

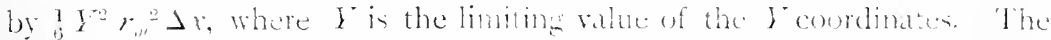

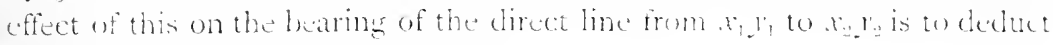
$\Delta+y^{2}$. We shall therefore hale

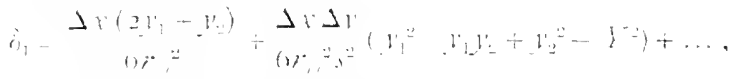

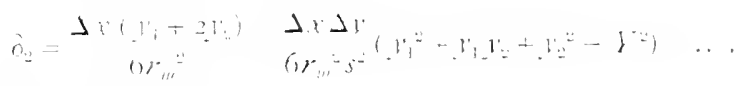

similarly we shall hat

or

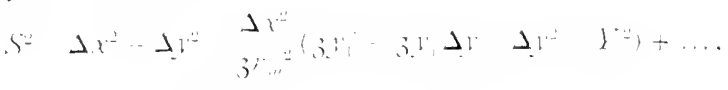

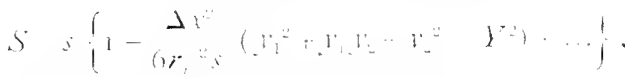

tending to reluee the errors in benth canen. 
If, instead of making the average scale error a minimum, we make the maximum scale error at the extreme meridian $Y$ equal and opposite to the scale error along the prinoipal meridian, as in the International Map on the scale of I-Million, we have only to replace the $Y^{2}$ in the above formulae by $Y^{2}$ to obtain the corresponding finite errors of bearing and distance.

The expressions can also be written in the form

$$
\begin{aligned}
& \delta_{1}=\frac{\mathrm{I}}{6 r_{m}^{2}}\left\{x\left(2 y_{1}+y_{2}\right)+\left(y_{1}^{2}+y_{1} y_{2}+y_{2}^{2}-Y^{2}\right) \sin t_{1} \cos t_{1} \ldots\right\}, \\
& \delta_{2}=\frac{1}{6 r_{m}^{2}}\left\{x\left(y_{1}+2 y_{2}\right)-\left(y_{1}^{2}+y_{1} y_{2}+y_{2}^{2}-Y^{2}\right) \sin t_{1} \cos t_{1} \ldots\right\}, \\
& S=s\left\{\mathrm{I}-\frac{\mathrm{I}}{6 r_{m}^{2}}\left(y_{1}{ }^{2}+y_{1} y_{2}+y_{2}^{2}-Y^{2}\right) \cos ^{2} t_{1} \ldots\right\} .
\end{aligned}
$$

lor the orthomorphic projections they reduce to

$$
\begin{aligned}
& \delta_{1}=\frac{\Delta x}{6 r_{1 m^{2}}{ }^{2}}\left(2 y_{1}+y_{2}\right)+\text { terms in } 4 \text { th powers of coordinates, } \\
& \delta_{2}=\frac{\Delta x}{6 r_{11}}\left(y_{1}+2 y_{2}\right)+, \quad, \quad, \quad, \quad, \quad, \\
& S-s\left\{1-\frac{1}{6 r_{11}{ }^{2}}\left(y_{1}^{2}+y_{1} y_{2}+y_{2}^{2}-Y^{2}\right) \ldots\right\},
\end{aligned}
$$

remembering that $\delta_{1}$ and $\delta_{22}$ will be in circular measure, and that if we want the scale error along the principal meridian equal and opposite to that along the extreme meridian we must write $\frac{3}{2} Y^{\prime \prime}$ instead of $Y^{*}$.

Pizzetti on p. 39 I gives formulae for the error in distance and bearing of a line, on the Direct Conical Orthomorphic Projection, starting from the mid-parallel with azimuth $a_{0}$, which appear to be erroneous. For $S-s$ he gives $\frac{s^{\prime \prime} \cos ^{2} \alpha_{0}}{2 p_{0}-V_{0}}$ which should read $\frac{s^{3} \cos ^{2} \alpha_{0}}{6 \rho_{0} \gamma_{0}}$ to agree with the above (putting $\hat{y}_{1}=0$ and $y_{2} \equiv x_{2}$ in direct system $\left.\equiv s \cos t_{3}\right)$. For $\delta_{1}$ he gives $\epsilon=\frac{s^{2} \cos ^{2} u_{11}}{6 p_{n} \nu_{11}}$ which should read $\frac{s^{2} \sin a_{01} \cos a_{t 1}}{6 \rho_{01} N_{0}}$.

\section{An error in Jordan's Handbuch.}

In checking the formulae above the writer had occasion to use the formulate for Reversion of Series given by Jordan on p. 191, and in doing so found an error in the third line of terms for the value of $x$ printed thus:

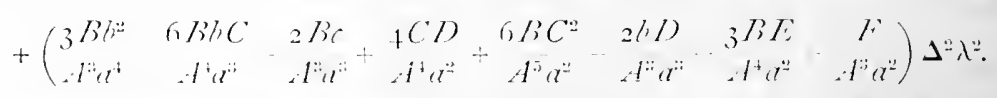

The coefficient of the fourth term is in crror. It should be $\frac{3 C D}{2 d^{4}}$ instcat of $\begin{aligned} & +C D \\ & A^{4} q^{2}\end{aligned}$ 


\section{CHAPTER VI}

\section{THE CONVERGENCY OF MERIIANS}

The quéstion of Convergency of Meridians has always presented some difficulty to the writer, and probably to others atso, especially when applied to the spheroid. Jordan gives two or three definitions of it in different parts of Vol. 11 of his Handbuch, and yet another clefinition at p. 501 derived from Gauss when dealing with the Conformal Projection. 'This latter he distingushes by calling it the Convergency of Map Neridians, being the angle between the tangent at a point of a projected meridian of the spheroid and the principal meridian of the map. 'This of course is the convergency which a user of the map or projection wants to know when converting a bearing calculated from the coordinates into a true bearing. It will be noted however that the convergency so derived differs from the convergency as derived from the Cassini P'rojection by a small quantity $=\frac{2}{-3} \lambda^{3} \eta^{2} \sin \phi \cos ^{2} \phi$. An explanation of this difference is giren by Jordan on pip. 5०3-4 which for some time puzzled the writer and it was not until he had studied the work of J. I. Craig on the theory of the indicatrix that the explanation became clear.

It might appear that the $x$ coordinate in the Gauss Conformal Projection was the same as in the Cassini. It is so in the case of the sphere, but not in the case of the spheroid. By expanding the formulac for the coordinates on the Cassini after the manner of Jordan still further the writer finds

$$
\begin{aligned}
& x=\mu+\frac{1}{2} v^{2} \sin \phi \cos \phi+\because \frac{1}{2} \lambda^{4} \sin \phi \cos ^{3} \phi\left(5-t^{2}+5 \eta^{2}\right)+\ldots,
\end{aligned}
$$

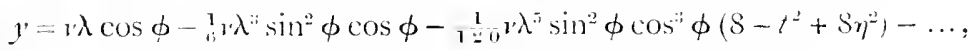

whereas on the (auss Conformal we have (Jordan, p. 497 )

$$
\begin{aligned}
& x=M+1 \lambda^{2} \sin \phi \cos \phi+\frac{1}{4} r^{4} \sin \phi \cos ^{\prime \prime} \phi\left(5-t^{2}+9 y^{2}\right)+\ldots, \\
& y=v^{\prime} \lambda \cos \phi+i_{i}^{1} \lambda^{\prime \prime} \cos ^{3} \phi\left(1-t^{\prime \prime}+\eta^{\prime \prime}\right)
\end{aligned}
$$

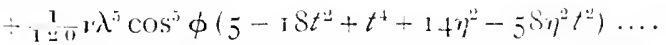

It will be observed that in the term in $\lambda^{4}$ for $x^{2} w_{e}$ have $+5 y^{2}$ in the cassini as against $+9 \eta^{2}$ in the craluss.

The following value for the convergency on the Cassint is also foumd by continuing the method of fordan :

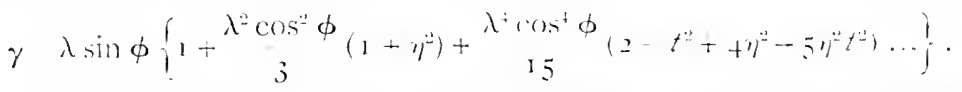

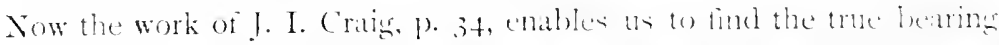
of the line on the spheroid to which al line, through any lome on the projection, prarallel to the axis of $x$ comesponels. It is

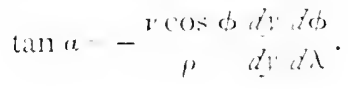


Performing these differentiations we find

$$
\begin{aligned}
& d y^{\prime} / d \phi=-\rho \lambda \sin \phi\left\{\mathrm{I}+\frac{\lambda^{2} \cos ^{2} \phi}{6}\left(2-t^{2}+2 \eta^{2}\right)\right. \\
& \left.+\frac{\lambda^{4} \cos ^{4} \phi}{\text { I } 20}\left(16-28 t^{2}+t^{4}+32 \eta^{2}-60 \eta^{2} t^{2}\right) \ldots\right\}, \\
& d y / d \lambda=+\nu \cos \phi\left\{\mathrm{I}-\lambda_{2}^{2} \cos ^{2} \phi t^{2}-\frac{\lambda^{4} \cos ^{4} \phi}{24}\left(8 t^{2}-t^{4}+8 \eta^{2} t^{2}\right) \ldots\right\},
\end{aligned}
$$

whence

$$
\begin{aligned}
\tan \alpha & =\lambda \sin \phi\left\{\begin{array}{l}
\int_{1}+\cos ^{2} \phi \\
3
\end{array}\left(\mathrm{I}+t^{2}+\eta^{2}\right)\right. \\
& \left.+{ }^{\lambda^{4} \cos ^{4} \phi}-{ }_{15}\left(2+4 t^{2}+2 t^{4}+4 \gamma^{2}\right) \ldots\right\}, \\
\alpha & =\lambda \sin \phi\left\{\mathrm{I}+\frac{\lambda^{2} \cos ^{2} \phi}{3}\left(\mathrm{I}+\eta^{2}\right)+\frac{\lambda^{4} \cos ^{4} \phi}{15}\left(2-t^{2}+4 \eta^{2}-5 \eta^{2} t^{2}\right) \ldots\right\},
\end{aligned}
$$

agreeing with the convergency.

When however we apply the same method to the Causs Conformal Projection to find the true bearing on the spheroid to which a line of zero bearing on the projection corresponds we shall find

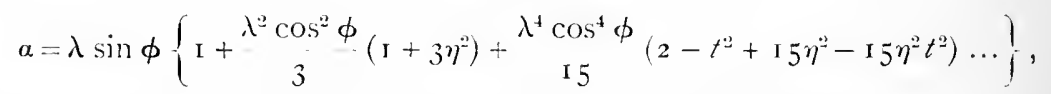

differing from the value in the Cassini ly the small quantity

$$
\because \eta^{\prime \prime} \lambda^{*} \sin \phi \cos ^{2} \phi+\ldots
$$

This shews that in the Conformal System the lines of zero bearing do not correspond with the lines of zero bearing in the Cassini System, or with the lines of same bearing on the spheroid. The indicatrix, though a circle in the Conformal System, is at every point turned through the above small angle. This is a point not of practical importance perhaps, as the quantity in question is so small, but it has some theoretical interest in understanding the true nature of the comvergency of map meridians.

For the scale value along a line parallel to the principal meridian on the projection we also find from I. I. Craig's formulae

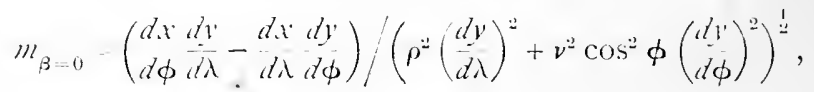

which in the case of the Cassini leads to

$$
m_{\beta-10} 1-\frac{1}{2} \lambda \cos ^{2} \phi\left(1+\eta^{2}\right)+2 \cos ^{4} \phi\left(5-\cos ^{2} \phi+10 \eta^{2}-2+\eta^{2} t^{2}\right)+\ldots
$$

whereas in the Couss Conformal we have

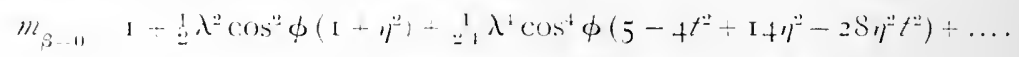

For any other value of $\beta$ we have approximately on the (assini

$$
m_{\beta} \quad 1: 2 \lambda^{2} \cos ^{2} \phi\left(1+\eta^{2}\right) \cos ^{2} \beta+\ldots
$$




\section{Some small errors in Helmert's Formulae for Convergency, etc.}

In checking the formulac for the convergency on the spheroid, ete. by Helmert's values the writer was led to discover some small errors in the latter, which should be pointed out, though they will hardly have any appreciable effect in practice.

It will be found that the value for the convergency can be put into the form

$\tan \gamma=\tan \lambda \sin \phi\left\{1+\frac{1}{3} \eta^{2} \cos ^{2} \phi \lambda^{2}+\int_{1: 2}^{1} \eta^{2} \cos ^{4} \phi \lambda^{4}\left(7 \quad 5^{t^{2}}\right)+\ldots i\right.$.

Transferring this into Helmert's form, we write

$$
\gamma \equiv t, \quad \lambda \equiv L_{1,2}, \quad \phi \equiv h_{2}, \quad \eta^{2}=\begin{gathered}
i^{2} \cos ^{2} B_{2} \\
\mathbf{1} \cdots i^{2}
\end{gathered},
$$

whence we have

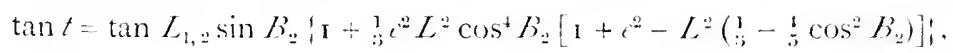

as against

$\tan t=\tan L_{1,2} \sin B_{2} i_{1}+1_{3}^{1} e^{2} L^{2} \cos ^{+} B_{2}\left[1+\frac{1}{2} e^{2}\left(5-3 \cos B_{2}\right)\right.$

as given by Helmert, p. $4+6$, equation ( 7 ).

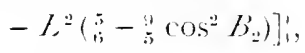

The error seems to arise in the equations (2), (4), (5) and (6) on the previous page +45 . In equation (2) the fourth line should read

$$
7 L^{4}\left(30-25 \sin ^{2} F^{4} \cos ^{2} F-16 \cos ^{4} F\right) \text {, }
$$

instead of $\quad \therefore L^{4}\left(30-10 \sin ^{2} F \mathrm{Cos}^{2} F-\cos ^{4} F\right)$.

Equations $(f)$ and $(5)$ are in error because the terms in $e^{2}$ are omitterl. though they are correctly given in efpuation (10), p. $4+2$, for the relation between $\cos F$ and $\cos B_{2}$. These omissions affect equation (6) and the following expation which should read

$$
\begin{aligned}
& \sin \beta_{2}=\sin B_{2}\left(1-\frac{1}{2} e^{22} \cos ^{2} F\left[1+\frac{1}{4} e^{2}\left(1-3 \sin ^{2} F\right)+L^{2} \sin ^{2} F\right.\right. \\
& +\frac{1}{2} C^{2} L^{2} \sin ^{2} F\left(\mathrm{r}+\sin ^{2} F\right)-\frac{1}{;} L^{4} \sin ^{2} F+L^{4} \sin ^{4} F \\
& \left.+1 e^{+4}\left(\mathrm{I}+2 \sin ^{2} F+5 \sin ^{+} F\right)\right\} \text {, }
\end{aligned}
$$

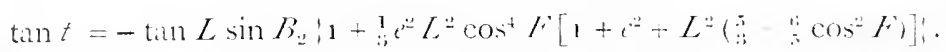

Helmert in incstigation is extremely difficult to follow and the use of $B$. the actual latitute of a point and $F$ the latitude of the print where the perpendicular coordinate of the irst point cuts the principal merklian, in the same formula, is very apt to lead to confusion.

On the sphere the latitude of the foot of the perpendiculat whith determines the $x$ coordinate in the Cassini l'rojection is griven hy

$$
\tan F=\tan P_{-,} \text {sec } L
$$

in Helmert's notation, or tan $\phi_{1}=\tan \phi \sec \lambda$ in ours. From this the weil known expansion for $\phi_{1}-\phi$ is derived by trigonometry for the sherrid the ame formula can be put in the form

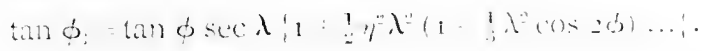


In the same way the formula for the $y$ coordinate can be put in the form $\sin \left(y / \nu_{\phi}\right)=\sin \lambda \cos \phi\left(1-\frac{1}{15} \eta^{2} \lambda^{4} \sin ^{2} \phi \cos ^{2} \phi\right)$.

\section{List of Expansions used in these Papers.}

$$
\begin{aligned}
& \sin \theta=\theta-\frac{1}{6} \theta^{3}+\frac{1}{1: \overline{0}} \theta^{5}-\frac{1}{50+0} \theta^{\pi}+\frac{1}{36 \Sigma^{2} \times 8 \overline{0}} \theta^{9}-\ldots, \\
& \cos \theta=1-\frac{1}{2} \theta^{2}+\frac{1}{21} \theta^{4}-\frac{1}{2} \overline{0}^{6}+\frac{1}{40} \frac{1}{2} \overline{0} \theta^{8}-\ldots, \\
& \tan \theta=\theta+\frac{1}{35} \theta^{* 3}+\frac{2}{15} \theta^{5}+\frac{17}{315} \theta^{7}+\frac{62}{25} \theta^{9}+\ldots, \\
& \cot \theta=\frac{\mathrm{I}}{\theta}\left\{\mathrm{I}-\frac{1}{i j} \theta^{2}-\frac{1}{45} \theta^{4}-\frac{2}{95} \theta^{6}-\ldots\right\}, \\
& \sec \theta=\mathbf{I}+\frac{1}{2} \theta^{2}+\frac{5}{2} \theta^{4}+\frac{61}{7} 2 \frac{1}{0} \theta^{6}+\frac{25}{80} \theta^{8}+\ldots, \\
& \operatorname{cosec} \theta=\frac{1}{\theta}\left\{1+\frac{1}{5} \theta^{2}+\frac{7}{60} \theta^{4}+\frac{3}{15120} \theta^{6}+\ldots\right\}, \\
& \sin ^{-1} \theta=\theta+\frac{1}{6} \theta^{3}+\frac{3}{40} \theta^{5}+\frac{5}{11} \theta^{7}+\frac{35}{115} \theta^{9}+\ldots, \\
& \tan ^{-1} \theta=\theta-\frac{1}{i ;} \theta^{3}+\frac{1}{3} \theta^{5}-\frac{1}{7} \theta^{7}+\frac{1}{i 3} \theta^{9}-\ldots, \\
& \log _{e} \sin \theta=\log \theta-\frac{1}{6} \theta^{2}-\frac{1}{180} \theta^{4}-\frac{1}{385,5} \theta^{6}-\frac{1}{3 i} \frac{1}{1500} \theta^{8}-\ldots \text {, } \\
& \log _{e} \sec \theta=\frac{1}{2} \theta^{2}+{ }_{1: 2}^{1} \theta^{4}+\frac{1}{15} \theta^{6}+\frac{1}{25} \overline{5} \theta^{8}+\ldots,
\end{aligned}
$$

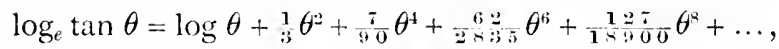

$$
\begin{aligned}
& \log _{e}(\mathrm{r}+\theta)=\theta-\frac{1}{2} \theta^{*}+\frac{1}{3} \theta^{*}-\frac{1}{4} \theta^{4}+\frac{1}{3} \theta^{5}-\ldots, \\
& \log _{e}(\mathrm{I}-\theta)=-\left\{\theta+\frac{1}{2} \theta^{2}+\frac{1}{3 ;} \theta^{3}+\frac{1}{4} \theta^{4}+\frac{1}{5} \theta^{5}+\ldots\right\} \text {, } \\
& \log _{e} \frac{\mathrm{I}+\theta}{\mathrm{I}-\theta}=2\left\{\theta+\frac{1}{;} \theta^{3}+\frac{1}{5} \theta^{5}+\frac{1}{i} \theta^{\tau}+-\ldots\right\} \text {, } \\
& \tan (\theta+h)=\tan \theta+h\left(\mathrm{I}+t^{2}\right)+h^{2} t\left(\mathrm{I}+t^{2}\right)+\frac{1}{3} h^{3}\left(\mathrm{I}+4 t^{2}+3 t^{4}\right)+\ldots,
\end{aligned}
$$

where $t=\tan \theta$. 


\section{UCLA MAP LIBRARY}

University of California

Reference

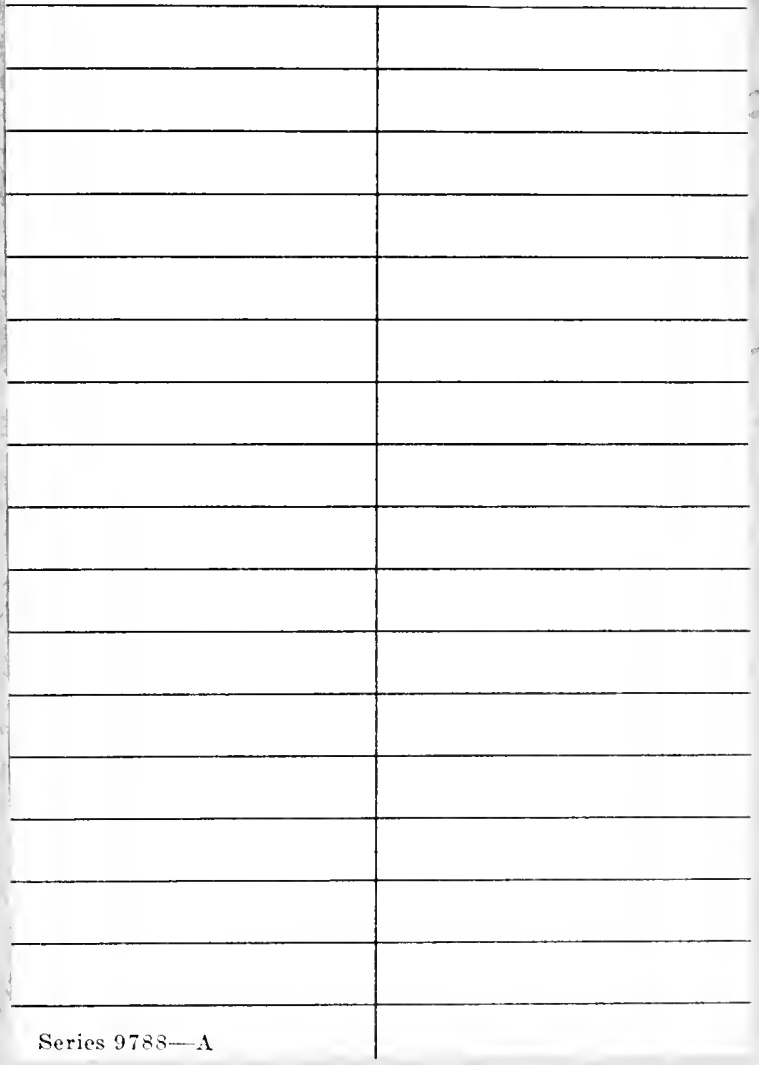


